\title{
On a differential inequality for equations of a viscous compressible heat conducting fluid bounded by a free surface
}

by EwA ZADRZYŃSKA and WoJciech M. ZAJA̧CZKOWsKi (Warszawa)

Abstract. We derive a global differential inequality for solutions of a free boundary problem for a viscous compressible heat conducting fluid. The inequality is essential in proving the global existence of solutions.

1. Introduction. The aim of this paper is to derive a global differential inequality for the following free boundary problem for a viscous compressible heat conducting fluid (see [2], Chs. 2 and 5):

$$
\begin{array}{ll}
\varrho\left[v_{t}+(v \cdot \nabla) v\right]+\nabla p-\mu \Delta v-\nu \nabla \operatorname{div} v=\varrho f & \text { in } \widetilde{\Omega}^{T}, \\
\varrho t_{t}+\operatorname{div}(\varrho v)=0 & \text { in } \widetilde{\Omega}^{T}, \\
\varrho c_{v}\left(\theta_{t}+v \cdot \nabla \theta\right)+\theta p_{\theta} \operatorname{div} v-\kappa \Delta \theta & \\
\quad \quad-\frac{1}{2} \mu \sum_{i, j=1}^{3}\left(v_{i, x_{j}}+v_{j, x_{i}}\right)^{2}-(\nu-\mu)(\operatorname{div} v)^{2}=\varrho r & \text { in } \widetilde{\Omega}^{T}, \\
\mathbb{T} \bar{n}=-p_{0} \bar{n} & \text { on } \widetilde{S}^{T}, \\
v \cdot \bar{n}=-\phi_{t} /|\nabla \phi| & \text { on } \widetilde{S}^{T}, \\
\partial \theta / \partial n=\theta_{1} & \text { on } \widetilde{S}^{T}, \\
\left.v\right|_{t=0}=v_{0},\left.\quad \varrho\right|_{t=0}=\varrho_{0},\left.\quad \theta\right|_{t=0}=\theta_{0} & \text { in } \Omega,
\end{array}
$$

where $\widetilde{\Omega}^{T}=\bigcup_{t \in(0, T)} \Omega_{t} \times\{t\}, \Omega_{t}$ is a bounded domain of the drop at time $t$ and $\Omega_{0}=\Omega$ is its initial domain, $\widetilde{S}^{T}=\bigcup_{t \in(0, T)} S_{t} \times\{t\}, S_{t}=\partial \Omega_{t}$, $\phi(x, t)=0$ describes $S_{t}$, and $\bar{n}$ is the unit outward vector normal to the boundary (i.e. $\bar{n}=\nabla \phi /|\nabla \phi|)$.

1991 Mathematics Subject Classification: 35A05, 35R35, 76N10.

Key words and phrases: free boundary, compressible viscous heat conducting fluid. 
Moreover, $v=v(x, t)$ is the velocity of the fluid, $\varrho=\varrho(x, t)$ the density, $\theta=\theta(x, t)$ the temperature, $f=f(x, t)$ the external force field per unit mass, $r=r(x, t)$ the heat sources per unit mass, $\theta_{1}=\theta_{1}(x, t)$ the heat flow per unit surface, $p=p(\varrho, \theta)$ the pressure, $\mu$ and $\nu$ the viscosity coefficients, $\kappa$ the coefficient of heat conductivity, $c_{v}=c_{v}(\varrho, \theta)$ the specific heat at constant volume, and $p_{0}$ the external (constant) pressure.

We assume that $c_{v}>0$, the coefficients $\mu, \nu, \kappa$ are constants, and $\kappa>0$, $\nu \geq \mu>0$.

Finally, $\mathbb{T}=\mathbb{T}(v, p)$ denotes the stress tensor of the form

$$
\begin{aligned}
\mathbb{T}=\left\{T_{i j}\right\} & =\left\{-p \delta_{i j}+\mu\left(v_{i, x_{j}}+v_{j, x_{i}}\right)+(\nu-\mu) \delta_{i j} \operatorname{div} v\right\} \\
& \equiv\left\{-p \delta_{i j}+D_{i j}(v)\right\},
\end{aligned}
$$

where $i, j=1,2,3$, and $\mathbb{D}=\mathbb{D}(v)=\left\{D_{i j}\right\}$ is the deformation tensor. Let the domain $\Omega$ be given. Then by $(1.1)_{5}, \Omega_{t}=\left\{x \in \mathbb{R}^{3}: x=x(\xi, t), \xi \in \Omega\right\}$, where $x=x(\xi, t)$ is the solution of the Cauchy problem

$$
\frac{\partial x}{\partial t}=v(x, t),\left.\quad x\right|_{t=0}=\xi \in \Omega, \xi=\left(\xi_{1}, \xi_{2}, \xi_{3}\right) .
$$

Therefore, the transformation $x=x(\xi, t)$ connects the Eulerian $x$ and the Langrangian $\xi$ coordinates of the same fluid particle. Hence

$$
x=\xi+\int_{0}^{t} u(\xi, s) d s \equiv X_{u}(\xi, t),
$$

where $u(\xi, t)=v\left(X_{u}(\xi, t), t\right)$. Moreover, the kinematic boundary condition $(1.1)_{5}$ implies that the boundary $S_{t}$ is a material surface. Thus, if $\xi \in S=S_{0}$ then $X_{u}(\xi, t) \in S_{t}$ and $S_{t}=\left\{x: x=X_{u}(\xi, t), \xi \in S\right\}$.

By the continuity equation $(1.1)_{2}$ and $(1.1)_{5}$ the total mass of the drop is conserved and the following relation holds between $\varrho$ and $\Omega_{t}$ :

$$
\int_{\Omega_{t}} \varrho(x, t) d x=M .
$$

This paper is divided into three sections. In Section 2 we introduce some notation. In Section 3 we derive the main result of the paper, i.e. the differential inequality (3.160) (see Theorem 3.13) which is essential in proving the global existence of a solution of problem (1.1) (see [19]). In order to obtain inequality (3.160) we impose the following assumptions:

a) there exists a sufficiently smooth local solution;

b) the transformation (1.2) together with its inverse exist;

c) the volume and the shape of the domain do not change much in time.

Papers concerning problem (1.1) include [15]-[17] and [20]. In [15] the local-in-time existence and uniqueness of solution to problem (1.1) in the 
Sobolev-Slobodetskiı spaces is proved. In [17] we prove that under an appropriate choice of $\varrho_{0}, v_{0}, \theta_{0}, \theta_{1}, p_{0}, \kappa$ and the form of the internal energy per unit mass $\varepsilon=\varepsilon(\varrho, \theta), \operatorname{var}_{t}\left|\Omega_{t}\right|$ is as small as we need. Paper [20] contains the global existence theorem for problem (1.1). In [15], [18], [19], [21] we consider the motion of a viscous compressible heat conducting fluid bounded by a free surface governed by surface tension. Such a motion is described by equations $(1.1)_{1}-(1.1)_{3}$ with conditions $(1.1)_{5}-(1.1)_{7}$ and with the condition

$$
\mathbb{T} \bar{n}-\sigma H \bar{n}=-p_{0} \bar{n}
$$

replacing $(1.1)_{4}$. In (1.3), $\sigma$ is the constant coefficient of surface tension, and $H$ is the double mean curvature of $S_{t}$.

Similarly to the case $\sigma=0$, in [15] the local motion of a capillary fluid (the case $\sigma \neq 0$ ) is considered, while [18], [19] and [21] give, in that case, analogous to those of [17], the present paper and [20], respectively. In [18] conservation laws and global estimates for equations $(1.1)_{1}-(1.1)_{3}$ with conditions (1.3) and (1.1) $)_{5}-(1.1)_{7}$ are presented. Moreover, we prove in [18] that we can choose $\varrho_{0}, v_{0}, \theta_{0}, \theta_{1}, p_{0}, \kappa, \sigma$ and the form of the internal energy per unit mass $\varepsilon=\varepsilon(\varrho, \theta)$ such that $\operatorname{var}_{t}\left|\Omega_{t}\right|$ is as small as we need. This result is used in [21] to prove the global-in-time existence of solutions to problem $(1.1)_{1}-(1.1)_{3},(1.3),(1.1)_{5}-(1.1)_{7}$. Paper [19] is devoted to a differential inequality for problem $(1.1)_{1}-(1.1)_{3},(1.3),(1.1)_{5}-(1.1)_{7}$ which is analogous to inequality (3.160). In [21] the global existence theorem for problem $(1.1)_{1}{ }^{-}$ $(1.1)_{3},(1.3),(1.1)_{5}-(1.1)_{7}$ is proved. Finally, [16] contains the review of all results from [17]-[21] including the main result proved in this paper.

The motion of a viscous compressible heat conducting fluid in a fixed domain was considered by A. Matsumura and T. Nishida [3]-[7], A.Valli [13], and A. Valli and W. M. Zajączkowski [14]. Papers [3] and [4] are concerned with the initial value problem for equations $(1.1)_{1}-(1.1)_{3}$ considered in $\mathbb{R}^{3} \times(0, \infty)$. In [4] the existence and uniqueness of a global-in-time classical solution of system $(1.1)_{1}-(1.1)_{3}$ is proved for the initial conditions

$$
\left.v\right|_{t=0}=v_{0},\left.\quad \varrho\right|_{t=0}=\varrho_{0},\left.\quad \theta\right|_{t=0}=\theta_{0} \quad \text { in } \mathbb{R}^{3} .
$$

The solution is obtained in a neighbourhood of a constant state $(v, \varrho, \theta)=$ $(0, \bar{\varrho}, \bar{\theta})$, where $\bar{\varrho}$ and $\bar{\theta}$ are positive constants. In [3] the same type of result is obtained for a polytropic gas, i.e. under the assumption that $\varepsilon=c_{v} \theta$, where $\varepsilon$ is the internal energy. In [7] the global existence theorem is proved for system $(1.1)_{1}-(1.1)_{3}$ considered in $\Omega \times(0, \infty)$ (where $\Omega$ is a halfspace or an exterior domain of any bounded region with smooth boundary) with initial conditions (1.4) and with the boundary conditions of Dirichlet or Neumann type. Papers [5], [6], [13] and [14] are concerned with the global motion of a viscous compressible heat conducting fluid in a bounded domain $\Omega \subset \mathbb{R}^{3}$.

For a compressible barotropic fluid (i.e. when the temperature of the 
fluid is constant) the problem corresponding to (1.1) has been examined by W. M. Zajączkowski [22]-[25] and V. A. Solonnikov and A. Tani [12]. In [23]-[24] the local motion of a compressible barotropic fluid bounded by a free surface is considered, while [22], [25] and [12] are devoted to the global motion of such a fluid.

In [8] K. Pileckas and W. M. Zajạczkowski proved the existence of a stationary motion of a viscous compressible barotropic fluid bounded by a free surface governed by surface tension.

Finally, papers of V. A. Solonnikov [9]-[11] concern free boundary problems for viscous incompressible fluids. In the case of an incompressible fluid $\varrho=$ const, so the continuity equation $(1.1)_{2}$ reduces to

$$
\operatorname{div} v=0 .
$$

Therefore, the problem examined by V. A. Solonnikov [9]-[11] is described by the Navier-Stokes equations $(1.1)_{1}$ (where $p=p(x, t)$ ) and by (1.5) with the initial condition $\left.v\right|_{t=0}=v_{0}$ and with the boundary condition being either $(1.1)_{4}$ or $(1.3)$.

2. Notation. Let $Q=\Omega_{t}$ or $Q=S_{t}(t \geq 0)$. By $\|\cdot\|_{l, Q}(l \geq 0)$ and $|\cdot|_{p, Q}(1 \leq p \leq \infty)$ we denote the norms in the usual Sobolev spaces $W_{2}^{l}(Q)$ and in the $L_{p}(Q)$ spaces, respectively.

Next, we introduce the space $\Gamma_{k}^{l}(\Omega)$ of functions $u$ with the norm

$$
\|u\|_{\Gamma_{k}^{l}(\Omega)}=\sum_{i \leq l-k}\left\|\partial_{t}^{i} u\right\|_{l-i, \Omega} \equiv|u|_{l, k, \Omega}, \quad \text { where } l>0, k \geq 0 .
$$

In the sequel we shall use the following notation for derivatives of $u$. If $u$ is a scalar-valued function we denote by $D_{x, t}^{k} u$ or $\underbrace{u_{x \ldots x t \ldots t}}_{k \text { times }}$ the vector
$\left(D_{x}^{\alpha} \partial_{t}^{i} u\right)_{|\alpha|+i=k}$.

Similarly, if $u=\left(u_{1}, u_{2}, u_{3}\right)$ we denote by $D_{x, t}^{k} u$ or $u_{\underbrace{x \ldots x t \ldots t}_{\text {times }}}$ the vector $\left(D_{x}^{\alpha} \partial_{t}^{i} u_{j}\right)_{|\alpha|+i=k, j=1,2,3}$. Hence $\left|D_{x, t}^{k} u\right|=\sum_{|\alpha|+i=k}\left|D_{x}^{\alpha} \partial_{t}^{i} u\right|$.

We use the following lemma.

LEMma 2.1. The following imbedding holds: $W_{r}^{l}(Q) \subset L_{p}^{\alpha}(Q)\left(Q \subset \mathbb{R}^{3}\right)$, where $|\alpha|+3 / r-3 / p \leq l, l \in \mathbb{Z}, 1 \leq p, r \leq \infty ; L_{p}^{\alpha}(\Omega)$ is the space of functions u such that $\left|D_{x}^{\alpha} u\right|_{p, \Omega}<\infty$, and $W_{r}^{l}(Q)$ is the Sobolev space.

Moreover, the following interpolation inequalities hold:

$$
\left|D_{x}^{\alpha} u\right|_{p, Q} \leq c \varepsilon^{1-\kappa}\left|D_{x}^{l} u\right|_{r, Q}+c \varepsilon^{-\kappa}|u|_{r, Q},
$$

where $\kappa=|\alpha| / l+3 /(l r)-3 /(l p)<1, \varepsilon$ is a parameter, and $c>0$ is a constant independent of $u$ and $\varepsilon$; and

$$
\left|D_{x}^{\alpha} u\right|_{q, \partial Q} \leq c \varepsilon^{1-\kappa}\left|D_{x}^{l} u\right|_{r, Q}+c \varepsilon^{-\kappa}|u|_{r, Q},
$$


where $\kappa=|\alpha| / l+3 /($ lr $)-2 /(l q)<1, \varepsilon$ is a parameter, and $c>0$ is a constant independent of $u$ and $\varepsilon$.

Lemma 2.1 follows from Theorem 10.2 of [1].

3. Global differential inequality. Assume that the existence of a sufficiently smooth local solution of problem (1.1) has been proved. To show the differential inequality we consider the motion near the constant state $v_{e}=0, p_{e}=p_{0}, \theta_{e}=\bar{\theta}_{0}=\frac{1}{|\Omega|} \int_{\Omega} \theta_{0} d \xi$ and $\varrho_{e}$, where $\varrho_{e}$ is a solution of the equation

$$
p\left(\varrho_{e}, \theta_{e}\right)=p_{0}
$$

Let

$$
p_{\sigma}=p-p_{0}, \quad \varrho_{\sigma}=\varrho-\varrho_{0}, \quad \vartheta_{0}=\theta-\theta_{e}, \quad \vartheta=\theta-\theta_{\Omega_{t}},
$$

where

$$
\theta_{\Omega_{t}}=\frac{1}{\left|\Omega_{t}\right|} \int_{\Omega_{t}} \theta d x .
$$

Then problem (1.1) takes the form

$$
\begin{array}{ll}
\varrho\left[v_{t}+(v \cdot \nabla) v\right]-\operatorname{div} \mathbb{T}\left(v, p_{\sigma}\right)=\varrho f & \text { in } \Omega_{t}, t \in[0, T], \\
\varrho \varrho_{t}+\operatorname{div}(\varrho v)=0 & \text { in } \Omega_{t}, t \in[0, T], \\
\varrho c_{v}(\varrho, \theta)\left(\vartheta_{0 t}+v \cdot \nabla \vartheta_{0}\right)+\theta p_{\theta}(\varrho, \theta) \operatorname{div} v & \\
\quad-\kappa \Delta \vartheta_{0}-\frac{1}{2} \mu \sum_{i, j}\left(\partial_{x_{i}} v_{j}+\partial_{x_{j}} v_{i}\right)^{2} & \\
\quad-(\nu-\mu)(\operatorname{div} v)^{2}=\varrho r & \text { in } \Omega_{t}, t \in[0, T], \\
\mathbb{T}\left(v, p_{\sigma}\right) \bar{n}=0 & \text { on } S_{t}, t \in[0, T], \\
\partial \vartheta_{0} / \partial n=\theta_{1} & \text { on } S_{t}, t \in[0, T],
\end{array}
$$

where $\mathbb{T}\left(v, p_{\sigma}\right)=\left\{\mu\left(\partial_{x_{i}} v_{j}+\partial_{x_{j}} v_{i}\right)+(\nu-\mu) \delta_{i j} \operatorname{div} v-p_{\sigma} \delta_{i j}\right\}$ and $T$ is the time of local existence.

In the sequel we shall use the following Taylor formula for $p_{\sigma}$ :

$$
\begin{aligned}
p_{\sigma}= & p(\varrho, \theta)-p\left(\varrho_{e}, \theta_{e}\right)=p(\varrho, \theta)-p\left(\varrho_{e}, \theta\right)+p\left(\varrho_{e}, \theta\right)-p\left(\varrho_{e}, \theta_{e}\right) \\
= & \left(\varrho-\varrho_{e}\right) \int_{0}^{1} p_{\varrho}\left(\varrho_{e}+s\left(\varrho-\varrho_{e}\right), \theta\right) d s \\
& +\left(\theta-\theta_{e}\right) \int_{0}^{1} p_{\theta}\left(\varrho_{e}, \theta_{e}+s\left(\theta-\theta_{e}\right)\right) d s \equiv p_{1} \varrho_{\sigma}+p_{2} \vartheta_{0} .
\end{aligned}
$$

We shall also use the formula 


$$
\begin{aligned}
p_{\sigma}= & p(\varrho, \theta)-p\left(\varrho_{\Omega_{t}}, \theta_{\Omega_{t}}\right) \\
= & \left(\varrho-\varrho_{\Omega_{t}}\right) \int_{0}^{1} p_{\varrho}\left(\varrho_{\Omega_{t}}+s\left(\varrho-\varrho_{\Omega_{t}}\right), \theta\right) d s \\
& +\left(\theta-\theta_{\Omega_{t}}\right) \int_{0}^{1} p_{\theta}\left(\varrho_{\Omega_{t}}, \theta_{\Omega_{t}}+s\left(\theta-\theta_{\Omega_{t}}\right)\right) d s \equiv p_{3} \bar{\varrho}_{\Omega_{t}}+p_{4} \vartheta,
\end{aligned}
$$

where the function $\varrho_{\Omega_{t}}=\varrho_{\Omega_{t}}(t)$ is a solution of the problem

$$
p\left(\varrho_{\Omega_{t}}, \theta_{\Omega_{t}}\right)=p_{0},\left.\quad \varrho_{\Omega_{t}}\right|_{t=0}=\varrho_{e}
$$

and

$$
\bar{\varrho}_{\Omega_{t}}=\varrho-\varrho_{\Omega_{t}} .
$$

The functions $p_{i}(i=1,2,3,4)$ in (3.4) and (3.5) are positive and $p_{1}=$ $p_{1}(\varrho, \theta), p_{2}=p_{2}\left(\varrho_{e}, \theta\right), p_{3}=p_{3}\left(\varrho_{\Omega_{t}}, \varrho, \theta\right), p_{4}=p_{4}\left(\varrho_{\Omega_{t}}, \theta_{\Omega_{t}}, \theta\right)$.

Now we point out the following facts concerning the estimates in Lemmas 3.1-3.12 and Theorem 3.13: them.

- By $\varepsilon$ we denote small constants and for simplicity we do not distinguish

- By $C_{1}$ and $C_{2}$ we denote constants which depend on $\varrho_{*}, \varrho^{*}, \theta_{*}, \theta^{*}, T$, $\int_{0}^{T}\|v\|_{3, \Omega_{t^{\prime}}}^{2} d t^{\prime},\|S\|_{4-1 / 2}$, on the parameters which guarantee the existence of the inverse transformation to $x=x(\xi, t)$ and also the constants of the imbedding theorems and the Korn inequalities. $C_{1}$ is always the coefficient of a linear term, while $C_{2}$ is the coefficient of a nonlinear term. For simplicity we do not distinguish different $C_{1}$ 's and $C_{2}$ 's.

- By $c$ we denote absolute constants which may depend on $\mu, \nu, \kappa$, and by $c_{0}<1$ we denote positive constants which may depend on $\mu, \nu, \kappa, \varrho_{*}$, $\varrho^{*}, \theta_{*}, \theta^{*}$. For simplicity we do not distinguish different $c$ 's and $c_{0}$ 's.

- We underline that all the estimates are obtained under the assumption that there exists a local-in-time solution of (1.1), so all the quantities $\varrho_{*}, \varrho^{*}, \theta_{*}, \theta^{*}, T, \int_{0}^{T}\|v\|_{3, \Omega_{t^{\prime}}}^{2} d t^{\prime},\|S\|_{4-1 / 2}$ are estimated by the data functions. Moreover, the existence of the inverse transformation to $x=x(\xi, t)$ is guaranteed by the estimates for the local solution (see [14]).

LEMma 3.1. Let $v, \varrho, \vartheta_{0}$ be a sufficiently smooth solution of (3.3). Then

$$
\begin{aligned}
& \frac{1}{2} \frac{d}{d t} \int_{\Omega_{t}}\left(\varrho v^{2}+\frac{p_{1}}{\varrho} \varrho_{\sigma}^{2}+\bar{\varrho}_{\Omega_{t}}^{2}+\frac{p_{2} \varrho c_{v}}{p_{\theta} \theta} \vartheta_{0}^{2}\right) d x \\
& \quad+c_{0}\|v\|_{1, \Omega_{t}}^{2}+(\nu-\mu)\|\operatorname{div} v\|_{0, \Omega_{t}}^{2}+c_{0}\left\|\vartheta_{0 x}\right\|_{0, \Omega_{t}}^{2}
\end{aligned}
$$




$$
\begin{aligned}
\leq & \varepsilon\left(\left\|p_{\sigma}\right\|_{0, \Omega_{t}}^{2}+\left\|\vartheta_{0 t x}\right\|_{0, \Omega_{t}}^{2}\right) \\
& +C_{1}\left(\|v\|_{0, \Omega_{t}}^{2}+\|r\|_{0, \Omega_{t}}^{2}+\|r\|_{0, \Omega_{t}}+\left\|\theta_{1}\right\|_{1, \Omega_{t}}^{2}+\left\|\theta_{1}\right\|_{1, \Omega_{t}}+\|f\|_{0, \Omega_{t}}^{2}\right) \\
& +C_{2}\left(\left\|\varrho_{\sigma}\right\|_{2, \Omega_{t}}^{4}+\left\|\varrho_{\Omega_{\Omega_{t}}}\right\|_{2, \Omega_{t}}^{4}+\|v\|_{2, \Omega_{t}}^{4}+\left\|\vartheta_{0}\right\|_{2, \Omega_{t}}^{4}\right)
\end{aligned}
$$

where $\varepsilon>0$ is sufficiently small.

Proof. Multiplying (3.3) 1 by $v$, integrating over $\Omega_{t}$ and using the continuity equation $(3.3)_{2}$ and (3.4) we obtain

(3.9) $\quad \frac{1}{2} \frac{d}{d t} \int_{\Omega_{t}} \varrho v^{2} d x+\frac{\mu}{2} E_{\Omega_{t}}(v)+(\nu-\mu)\|\operatorname{div} v\|_{0, \Omega_{t}}^{2}$

$$
-\int_{\Omega_{t}} p_{1} \varrho_{\sigma} \operatorname{div} v d x-\int_{\Omega_{t}} p_{2} \vartheta_{0} \operatorname{div} v d x=\int_{\Omega_{t}} \varrho f v d x
$$

where $E_{\Omega_{t}}(v)=\int_{\Omega_{t}} \sum_{i, j=1}^{3}\left(\partial_{x_{i}} v_{j}+\partial_{x_{j}} v_{i}\right)^{2} d x$.

By the continuity equation $(3.3)_{2}$, energy equation $(3.3)_{3}$ and condition $(3.3)_{5}$ we have

$$
\begin{aligned}
-\int_{\Omega_{t}} p_{1} \varrho_{\sigma} \operatorname{div} v d x & =\int_{\Omega_{t}} \frac{p_{1}}{\varrho} \varrho_{\sigma}\left(\varrho_{\sigma t}+v \cdot \nabla \varrho_{\sigma}\right) d x \\
& =\frac{1}{2} \frac{d}{d t} \int_{\Omega_{t}} \frac{p_{1} \varrho_{\sigma}^{2}}{\varrho} d x+I_{1}
\end{aligned}
$$

where

$$
\begin{aligned}
\left|I_{1}\right| \leq & \varepsilon\left(\left\|v_{x}\right\|_{0, \Omega_{t}}^{2}+\left\|\vartheta_{0 x}\right\|_{0, \Omega_{t}}^{2}\right)+C_{1}\left(\|r\|_{0, \Omega_{t}}^{2}+\left\|\theta_{1}\right\|_{1, \Omega_{t}}^{2}\right) \\
& +C_{2}\left(\left\|\varrho_{\sigma}\right\|_{1, \Omega_{t}}^{4}+\|v\|_{1, \Omega_{t}}^{2}\left\|\varrho_{\sigma}\right\|_{2, \Omega_{t}}^{2}+\|v\|_{1, \Omega_{t}}^{2}\left\|\vartheta_{0}\right\|_{2, \Omega_{t}}^{2}\right. \\
& \left.+\|v\|_{2, \Omega_{t}}^{2}\left\|\varrho_{\sigma}\right\|_{1, \Omega_{t}}^{2}+\left\|\varrho_{\sigma}\right\|_{2, \Omega_{t}}^{2}\left\|\varrho_{\sigma}\right\|_{1, \Omega_{t}}^{2}\right) .
\end{aligned}
$$

Next, dividing equation $(3.3)_{3}$ by $\theta \varrho_{\theta}$, multiplying the result by $p_{2} \vartheta_{0}$ and integrating over $\Omega_{t}$ we get

$$
\begin{aligned}
& \int_{\Omega_{t}} \frac{p_{2} \varrho c_{v}}{\theta p_{\theta}}\left(\partial_{t} \frac{\vartheta_{0}^{2}}{2}+v \cdot \nabla \frac{\vartheta_{0}^{2}}{2}\right) d x+\int_{\Omega_{t}} p_{2} \vartheta_{0} \operatorname{div} v d x-\int_{\Omega_{t}} \frac{p_{2} \kappa \Delta \vartheta_{0}}{\theta p_{\theta}} \vartheta_{0} d x \\
& \quad-\int_{\Omega_{t}} \frac{p_{2} \mu}{2 \theta p_{\theta}} \sum_{i, j}\left(\partial_{x_{i}} v_{j}+\partial_{x_{j}} v_{i}\right)^{2} \vartheta_{0} d x-\int_{\Omega_{t}} \frac{p_{2}(\nu-\mu)}{\theta p_{\theta}}(\operatorname{div} v)^{2} \vartheta_{0} d x \\
& =\int_{\Omega_{t}} \frac{p_{2} \varrho r}{\theta p_{\theta}} \vartheta_{0} d x .
\end{aligned}
$$

Hence applying the boundary condition $(3.3)_{5}$ we have 


$$
\begin{gathered}
\int_{\Omega_{t}} \frac{p_{2} \varrho c_{v}}{\theta p_{\theta}}\left(\partial_{t} \frac{\vartheta_{0}^{2}}{2}+v \cdot \nabla \frac{\vartheta_{0}^{2}}{2}\right) d x+\int_{\Omega_{t}} p_{2} \vartheta_{0} \operatorname{div} v d x+\int_{\Omega_{t}} \frac{p_{2} \kappa}{\theta p_{\theta}}\left|\vartheta_{0 x}\right|^{2} d x \\
=I_{2}+\int_{\Omega_{t}} \frac{p_{2} \varrho r}{\theta p_{\theta}} \vartheta_{0} d x+\int_{S_{t}} \frac{p_{2} \kappa}{\theta p_{\theta}} \theta_{1} \vartheta_{0} d x
\end{gathered}
$$

where

$$
\begin{aligned}
\left|I_{2}\right| \leq & \varepsilon\left(\left\|v_{x}\right\|_{0, \Omega_{t}}^{2}+\left\|\vartheta_{0 x}\right\|_{0, \Omega_{t}}^{2}\right) \\
& +C_{2}\left\|\vartheta_{0}\right\|_{1, \Omega_{t}}^{2}\left(\|v\|_{2, \Omega_{t}}^{2}+\left\|\varrho_{\sigma}\right\|_{2, \Omega_{t}}^{2}+\left\|\vartheta_{0}\right\|_{2, \Omega_{t}}^{2}\right) .
\end{aligned}
$$

Moreover,

$$
\begin{aligned}
\left|\int_{\Omega_{t}} \frac{p_{2} \varrho r}{\theta p_{\theta}} \vartheta_{0} d x\right| & \leq\left|\int_{\Omega_{t}} \frac{p_{2} \varrho r}{\theta p_{\theta}} \vartheta d x\right|+\left|\int_{\Omega_{t}} \frac{p_{2} \varrho r}{\theta p_{\theta}}\left(\theta_{\Omega_{t}}-\theta_{e}\right) d x\right| \\
& \leq \varepsilon\|\vartheta\|_{0, \Omega_{t}}^{2}+C_{1}\left(\|r\|_{0, \Omega_{t}}^{2}+\|r\|_{0, \Omega_{t}}\right)
\end{aligned}
$$

and

(3.16) $\left|\int_{S_{t}} \frac{p_{2} \kappa}{\theta p_{\theta}} \theta_{1} \vartheta_{0} d s\right| \leq \varepsilon\left(\|\vartheta\|_{0, \Omega_{t}}^{2}+\left\|\vartheta_{0 x}\right\|_{0, \Omega_{t}}^{2}\right)+C_{1}\left(\left\|\theta_{1}\right\|_{1, \Omega_{t}}^{2}+\left\|\theta_{1}\right\|_{1, \Omega_{t}}\right)$.

Next, using equations $(3.3)_{2},(3.3)_{3}$ and condition $(3.3)_{5}$ yields

$$
\int_{\Omega_{t}} \frac{p_{2} \varrho c_{v}}{\theta p_{\theta}}\left(\partial_{t} \frac{\vartheta_{0}^{2}}{2}+v \cdot \nabla \frac{\vartheta_{0}^{2}}{2}\right) d x=\frac{1}{2} \frac{d}{d t} \int_{\Omega_{t}} \frac{p_{2} \varrho c_{v}}{\theta p_{\theta}} \vartheta_{0}^{2} d x+I_{3}
$$

where

$$
\begin{gathered}
\left|I_{3}\right| \leq \varepsilon\left(\left\|v_{x}\right\|_{0, \Omega_{t}}^{2}+\left\|\vartheta_{0 x}\right\|_{0, \Omega_{t}}^{2}\right)+C_{1}\left(\|r\|_{0, \Omega_{t}}^{2}+\left\|\theta_{1}\right\|_{1, \Omega_{t}}^{2}\right) \\
+C_{2}\left\|\vartheta_{0}\right\|_{1, \Omega_{t}}^{2}\left(\left\|\vartheta_{0}\right\|_{2, \Omega_{t}}^{2}+\|v\|_{2, \Omega_{t}}^{2}+\left\|\varrho_{\sigma}\right\|_{2, \Omega_{t}}^{2}\right) .
\end{gathered}
$$

Taking into account (3.9)-(3.11), (3.13)-(3.18), using Lemma 5.2 of [21] and the Poincaré inequality

$$
\|\vartheta\|_{0, \Omega_{t}} \leq C_{1}\left\|\vartheta_{0 x}\right\|_{0, \Omega_{t}}
$$

we obtain for sufficiently small $\varepsilon$,

(3.20) $\frac{1}{2} \frac{d}{d t} \int_{\Omega_{t}}\left(\varrho v^{2}+\frac{p_{1} \varrho_{\sigma}^{2}}{\varrho}+\frac{p_{2} \varrho c_{v}}{\theta p_{\theta}} \vartheta_{0}^{2}\right) d x+c_{0}\|v\|_{1, \Omega_{t}}^{2}$

$$
\begin{aligned}
& +(\nu-\mu)\|\operatorname{div} v\|_{0, \Omega_{t}}^{2}+c_{0}\left\|\vartheta_{0 x}\right\|_{0, \Omega_{t}}^{2} \\
\leq & C_{1}\left(\|v\|_{0, \Omega_{t}}^{2}+\|r\|_{0, \Omega_{t}}^{2}+\|r\|_{0, \Omega_{t}}+\left\|\theta_{1}\right\|_{1, \Omega_{t}}^{2}+\|\theta\|_{1, \Omega_{t}}+\|f\|_{0, \Omega_{t}}^{2}\right) \\
& +C_{2}\left[\left\|\varrho_{\sigma}\right\|_{1, \Omega_{t}}^{2}\left(\left\|\varrho_{\sigma}\right\|_{1, \Omega_{t}}^{2}+\|v\|_{2, \Omega_{t}}^{2}\right)\right. \\
& \left.+\left\|\varrho_{\sigma}\right\|_{2, \Omega_{t}}^{2}\left(\|v\|_{1, \Omega_{t}}^{2}+\left\|\vartheta_{0}\right\|_{2, \Omega_{t}}^{2}\right)+\left\|\vartheta_{0}\right\|_{1, \Omega_{t}}^{2}\left(\left\|\vartheta_{0}\right\|_{2, \Omega_{t}}^{2}+\|v\|_{2, \Omega_{t}}^{2}\right)\right] .
\end{aligned}
$$


Finally, by $(3.3)_{2}$ and (3.7) we have

$$
\partial_{t} \bar{\varrho}_{\Omega_{t}}+v \cdot \nabla \bar{\varrho}_{\Omega_{t}}+\varrho \operatorname{div} v+\partial_{t} \varrho_{\Omega_{t}}=0,
$$

where in view of (3.6),

$$
\partial_{t} \varrho_{\Omega_{t}}=-\frac{p_{\theta_{\Omega_{t}}}}{p_{\varrho_{\Omega_{t}}}} \partial_{t} \theta_{\Omega_{t}} .
$$

Using the definition of $\theta_{\Omega_{t}}$ we calculate

$$
\begin{aligned}
\partial_{t} \theta_{\Omega_{t}}= & \frac{1}{\left|\Omega_{t}\right|} \int_{\Omega_{t}} \vartheta_{0 t} d x+\frac{1}{\left|\Omega_{t}\right|} \int_{\Omega_{t}} \theta \operatorname{div} v d x \\
& -\frac{1}{\left|\Omega_{t}\right|^{2}}\left(\int_{\Omega_{t}} \theta d x\right)\left(\int_{\Omega_{t}} \operatorname{div} v d x\right) .
\end{aligned}
$$

Consider now

$$
\begin{aligned}
\frac{1}{2} \frac{d}{d t} \int_{\Omega_{t}} \bar{\varrho}_{\Omega_{t}}^{2} d x= & -\int_{\Omega_{t}} \bar{\varrho}_{\Omega_{t}}^{2} \varrho \operatorname{div} v d x-\int_{\Omega_{t}} \bar{\varrho}_{\Omega_{t}} \partial_{t} \varrho_{\Omega_{t}} d x \\
& +\frac{1}{2} \int_{\Omega_{t}} \bar{\varrho}_{\Omega_{t}}^{2} \operatorname{div} v d x,
\end{aligned}
$$

where we have used equation (3.21). Since by $(3.3)_{3}$,

(3.25) $\quad\left\|\vartheta_{0 t}\right\|_{0, \Omega_{t}}^{2}$

$$
\begin{aligned}
\leq & \varepsilon\left\|\vartheta_{0 x t}\right\|_{0, \Omega_{t}}^{2}+C_{1}\left(\|r\|_{0, \Omega_{t}}^{2}+\left\|\theta_{1}\right\|_{1, \Omega_{t}}^{2}+\left\|v_{x}\right\|_{0, \Omega_{t}}^{2}+\left\|\vartheta_{0 x}\right\|_{0, \Omega_{t}}^{2}\right) \\
& +C_{2}\left(\|v\|_{1, \Omega_{t}}^{2}\left\|\vartheta_{0}\right\|_{2, \Omega_{t}}^{2}+\|v\|_{1, \Omega_{t}}^{4}+\left\|\varrho_{\sigma}\right\|_{2, \Omega_{t}}^{2}\left\|\vartheta_{0}\right\|_{2, \Omega_{t}}^{2}+\left\|\vartheta_{0}\right\|_{2, \Omega_{t}}^{4}\right),
\end{aligned}
$$

relations (3.22)-(3.24) give the estimate

$$
\begin{aligned}
\frac{1}{2} \frac{d}{d t} \int_{\Omega_{t}} \bar{\varrho}_{\Omega_{t}}^{2} d x \leq & \varepsilon\left(\left\|\bar{\varrho}_{\Omega_{t}}\right\|_{0, \Omega_{t}}^{2}+\left\|\vartheta_{0 t x}\right\|_{0, \Omega_{t}}^{2}\right) \\
& +C_{1}\left(\|r\|_{0, \Omega_{t}}^{2}+\left\|\theta_{1}\right\|_{1, \Omega_{t}}^{2}+\left\|v_{x}\right\|_{0, \Omega_{t}}^{2}+\left\|\vartheta_{0 x}\right\|_{0, \Omega_{t}}^{2}\right) \\
& +C_{2}\left(\|v\|_{1, \Omega_{t}}^{2}\left\|\vartheta_{0}\right\|_{2, \Omega_{t}}^{2}+\|v\|_{2, \Omega_{t}}^{4}+\left\|\bar{\varrho}_{\Omega_{t}}\right\|_{1, \Omega_{t}}^{4}\right. \\
& \left.+\left\|\varrho_{\sigma}\right\|_{2, \Omega_{t}}^{2}\left\|\vartheta_{0}\right\|_{2, \Omega_{t}}^{2}+\left\|\vartheta_{0}\right\|_{2, \Omega_{t}}^{4}\right) .
\end{aligned}
$$

By (3.5) and the Poincaré inequality (3.19) we have

$$
\left\|\bar{\varrho}_{\Omega_{t}}\right\|_{0, \Omega_{t}} \leq C_{1}\left(\left\|\vartheta_{0 x}\right\|_{0, \Omega_{t}}+\left\|\varrho_{\sigma}\right\|_{0, \Omega_{t}}\right) .
$$

The estimates (3.20), (3.26) and (3.27) imply (3.8).

Lemma 3.2. Let $v, \varrho, \vartheta_{0}$ be a sufficiently smooth solution of (3.3). Then 


$$
\begin{aligned}
\frac{1}{2} \frac{d}{d t} \int_{\Omega_{t}} & \left(\varrho v_{t}+\frac{p_{\sigma \varrho}}{\varrho} \varrho_{\sigma t}^{2}+\frac{\varrho c_{v}}{\theta} \vartheta_{0 t}^{2}\right) d x \\
& +c_{0}\left\|v_{t}\right\|_{1, \Omega_{t}}^{2}+(\nu-\mu)\left\|\operatorname{div} v_{t}\right\|_{0, \Omega_{t}}^{2}+c_{0}\left\|\vartheta_{0 t}\right\|_{1, \Omega_{t}}^{2} \\
\leq & \varepsilon\left(\|v\|_{1, \Omega_{t}}^{2}+\left\|\vartheta_{0 x}\right\|_{0, \Omega_{t}}^{2}\right) \\
& +C_{1}\left(|f|_{1,0, \Omega_{t}}^{2}+|r|_{1,0, \Omega_{t}}^{2}+\left|\theta_{1}\right|_{2,1, \Omega_{t}}^{2}\right)+C_{2} X_{1}^{2}\left(1+X_{1}\right),
\end{aligned}
$$

where $X_{1}=|v|_{2,1, \Omega_{t}}^{2}+\left|\varrho_{\sigma}\right|_{2,1, \Omega_{t}}^{2}+\left|\vartheta_{0}\right|_{2,1, \Omega_{t}}^{2}$.

Proof. Differentiating (3.3) 1 with respect to $t$, multiplying by $v_{t}$ and integrating over $\Omega_{t}$ we obtain

$$
\begin{aligned}
& \quad \int_{\Omega_{t}}\left(\varrho \partial_{t} \frac{v_{t}^{2}}{2}+\varrho v \cdot \nabla \frac{v_{t}^{2}}{2}\right) d x+\frac{\mu}{2} E_{\Omega_{t}}\left(v_{t}\right)+(\nu-\mu)\left\|\operatorname{div} v_{t}\right\|_{0, \Omega_{t}}^{2} \\
& -\int_{\Omega_{t}} p_{\varrho} \varrho_{\sigma t} \operatorname{div} v_{t} d x-\int_{\Omega_{t}} p_{\theta} \vartheta_{0 t} \operatorname{div} v_{t} d x \\
& \quad+\int_{\Omega_{t}}\left(\varrho_{t} v_{t}^{2}+\varrho_{t} v_{t} \cdot(v \nabla v)+\varrho v_{t} \cdot\left(v_{t} \nabla v\right)\right) d x+\int_{S_{t}}\left(\mathbb{T}\left(v, p_{\sigma}\right) n_{t}\right) \cdot v_{t} d s \\
& =\int_{\Omega_{t}} \partial_{t}(\varrho f) \cdot v_{t} d x,
\end{aligned}
$$

where we have used the boundary condition $(3.3)_{4}$.

The continuity equation $(3.3)_{2}$ yields

$$
\begin{aligned}
& -\int_{\Omega_{t}} p_{\varrho} \varrho_{\sigma t} \operatorname{div} v_{t} d x \\
& =\int_{\Omega_{t}}\left(\frac{p_{\varrho}}{\varrho} \varrho_{\sigma t} \varrho_{\sigma t t}+\frac{p_{\varrho}}{\varrho} \varrho_{\sigma t}^{2} \operatorname{div} v+\frac{p_{\varrho}}{\varrho} \varrho_{\sigma t} v_{t} \nabla \varrho_{\sigma}+\frac{p_{\varrho}}{\varrho} \varrho_{\sigma t} v \nabla \varrho_{\sigma t}\right) d x \\
& =\frac{1}{2} \frac{d}{d t} \int_{\Omega_{t}} \frac{p_{\varrho}}{\varrho} \varrho_{\sigma t}^{2} d x+I_{4},
\end{aligned}
$$

where

$$
\begin{aligned}
\left|I_{4}\right| \leq & \varepsilon\left(\left\|v_{t}\right\|_{1, \Omega_{t}}^{2}+\left\|\varrho_{\sigma t}\right\|_{0, \Omega_{t}}^{2}\right) \\
& +C_{2}\left[\left\|\varrho_{\sigma t}\right\|_{1, \Omega_{t}}^{2}\left(\left\|\varrho_{\sigma}\right\|_{2, \Omega_{t}}^{2}+\left\|\vartheta_{0 t}\right\|_{1, \Omega_{t}}^{2}+\left\|\varrho_{\sigma t}\right\|_{1, \Omega_{t}}^{2}+\left\|\vartheta_{0}\right\|_{2, \Omega_{t}}^{2}\right)\right] .
\end{aligned}
$$

Using (3.30), (3.31) and Lemma 5.3 of [21] we obtain from (3.29) the inequality 


$$
\begin{aligned}
\frac{1}{2} \frac{d}{d t} \int_{\Omega_{t}} & \left(\varrho v_{t}^{2}+\frac{p_{\varrho}}{\varrho} \varrho_{\sigma t}^{2}\right) d x \\
& \quad+c_{0}\left\|v_{t}\right\|_{1, \Omega_{t}}^{2}+(\nu-\mu)\left\|\operatorname{div} v_{t}\right\|_{0, \Omega_{t}}^{2}-\int_{\Omega_{t}} p_{\theta} \vartheta_{0 t} \operatorname{div} v_{t} d x \\
\quad \leq & \varepsilon\left\|\varrho_{\sigma t}\right\|_{0, \Omega_{t}}^{2}+C_{1}|f|_{1,0, \Omega_{t}}^{2}+C_{2} X_{1}^{2}\left(1+X_{1}\right)
\end{aligned}
$$

Dividing now $(3.3)_{3}$ by $\theta$, differentiating with respect to $t$, multiplying by $\vartheta_{0 t}$, integrating over $\Omega_{t}$ and next applying the Hölder and Young inequalities and the Sobolev lemma gives

$$
\begin{aligned}
\frac{1}{2} \frac{d}{d t} \int_{\Omega_{t}} \frac{\varrho c_{v}}{\theta} \vartheta_{0 t}^{2} d x+\int_{\Omega_{t}} p_{\theta} \vartheta_{0 t} \operatorname{div} v_{t} d x+\frac{\kappa}{\theta^{*}} \int_{\Omega_{t}}\left|\vartheta_{0 t x}\right|^{2} d x \\
\leq \varepsilon\left(\left\|\varrho_{\sigma t}\right\|_{0, \Omega_{t}}^{2}+\left\|v_{t}\right\|_{0, \Omega_{t}}^{2}+\left\|\vartheta_{0 t}\right\|_{0, \Omega_{t}}^{2}+\left\|\vartheta_{0 t x}\right\|_{0, \Omega_{t}}^{2}\right) \\
\quad+C_{1}\left(\|r\|_{0, \Omega_{t}}^{2}+\left\|r_{t}\right\|_{0, \Omega_{t}}^{2}+\left|\theta_{1}\right|_{2,1, \Omega_{t}}^{2}\right)+C_{2} X_{1}^{2}
\end{aligned}
$$

From the continuity equation $(3.3)_{2}$ it follows that

$$
\left\|\varrho_{\sigma t}\right\|_{0, \Omega_{t}}^{2} \leq C_{1}\|v\|_{1, \Omega_{t}}^{2}+C_{2}\|v\|_{1, \Omega_{t}}^{2}\left\|\varrho_{\sigma}\right\|_{2, \Omega_{t}}^{2} .
$$

Finally, adding inequalities (3.32)-(3.33) and using (3.25) and (3.34) we obtain (3.28).

Lemmas 3.1 and 3.2 imply

Lemma 3.3. Let $v, \varrho, \vartheta_{0}$ be a sufficiently smooth solution of (3.3). Then

$$
\begin{aligned}
& \text { 3.35) } \quad \frac{1}{2} \frac{d}{d t} \int_{\Omega_{t}} \varrho\left(v^{2}+v_{t}^{2}\right) d x+\frac{1}{2} \frac{d}{d t} \int_{\Omega_{t}} \frac{1}{\varrho}\left(p_{1} \varrho_{\sigma}^{2}+p_{\varrho} \varrho_{\sigma t}^{2}\right) d x \\
& \quad+\frac{1}{2} \frac{d}{d t} \int_{\Omega_{t}} \bar{\varrho}_{\Omega_{t}}^{2} d x+\frac{1}{2} \frac{d}{d t} \int_{\Omega_{t}} \frac{\varrho c_{v}}{\theta}\left(\frac{p_{2}}{p_{\theta}} \vartheta_{0}^{2}+\vartheta_{0 t}^{2}\right) d x+c_{0}\left(\|v\|_{1, \Omega_{t}}^{2}+\left\|v_{t}\right\|_{1, \Omega_{t}}^{2}\right) \\
& \quad+(\nu-\mu)\left(\|\operatorname{div} v\|_{0, \Omega_{t}}^{2}+\left\|\operatorname{div} v_{t}\right\|_{0, \Omega_{t}}^{2}\right) \\
& \quad+c_{0}\left(\left\|\vartheta_{0 x}\right\|_{0, \Omega_{t}}^{2}+\left\|\vartheta_{0 t}\right\|_{1, \Omega_{t}}^{2}\right) \\
& \leq \varepsilon\left\|p_{\sigma}\right\|_{0, \Omega_{t}}^{2}+C_{1}\left(\|v\|_{0, \Omega_{t}}^{2}+|r|_{1,0, \Omega_{t}}^{2}+\|r\|_{0, \Omega_{t}}+\left|\theta_{1}\right|_{2,1, \Omega_{t}}^{2}+\left\|\theta_{1}\right\|_{0, \Omega_{t}}+|f|_{1,0, \Omega_{t}}^{2}\right) \\
& \quad+C_{2}\left[\left\|\bar{\varrho}_{\Omega_{t}}\right\|_{2, \Omega_{t}}^{4}+X_{1}^{2}\left(1+X_{1}\right)\right],
\end{aligned}
$$

where $X_{1}$ is defined in Lemma 3.2.

In order to obtain an inequality for derivatives with respect to $x$ we rewrite problem (3.3) in the Lagrangian coordinates and next we introduce 
a partition of unity in the fixed domain $\Omega$. Thus we have

$$
\begin{aligned}
& \eta u_{i t}-\nabla_{u_{j}} T_{u}^{i j}\left(u, p_{\sigma}\right)=\eta g_{i}, \quad i=1,2,3, \\
& \eta_{\sigma t}+\eta \nabla_{u} \cdot u=0, \\
& \eta c_{v}(\eta, \Gamma) \gamma_{0 t}-\kappa \nabla_{u}^{2} \gamma_{0} \\
& \quad=\eta k-\Gamma p_{\Gamma}(\eta, \Gamma) \nabla_{u} \cdot u \\
& \quad+\frac{\mu}{2} \sum_{i, j=1}^{3}\left(\xi_{k x_{i}} \partial_{\xi_{k}} u_{j}+\xi_{k x_{j}} \partial_{\xi_{k}} u_{i}\right)^{2}+(\nu-\mu)\left(\nabla_{u} \cdot u\right)^{2}, \\
& \quad \mathbb{T}_{u}\left(u, p_{\sigma}\right) \bar{n}(\xi, t)=0, \\
& \bar{n} \cdot \nabla_{u} \Gamma=\Gamma_{1},
\end{aligned}
$$

where

$$
\begin{aligned}
& \eta(\xi, t)=\varrho(x(\xi, t), t), \quad u(\xi, t)=v(x(\xi, t), t), \quad g(\xi, t)=f(x(\xi, t), t), \\
& \Gamma(\xi, t)=\theta(x(\xi, t), t), \quad \gamma_{0}(\xi, t)=\vartheta_{0}(x(\xi, t), t), \quad \Gamma_{1}=\theta_{1}(x(\xi, t), t)
\end{aligned}
$$

and

$$
\begin{aligned}
& \mathbb{T}_{u}\left(u, p_{\sigma}\right)=\left\{T_{u}^{i j}\left(u, p_{\sigma}\right)\right\} \\
& \quad=\left\{-p_{\sigma} \delta_{i j}+\mu\left(\nabla_{u_{j}} u_{i}+\nabla_{u_{i}} u_{j}\right)+(\nu-\mu) \delta_{i j} \nabla_{u} \cdot u\right\} \\
& \nabla_{u}=\xi_{x} \partial_{\xi} \equiv\left(\xi_{i x_{k}} \partial_{\xi_{i}}\right)_{k=1,2,3}, \quad \nabla_{u_{i}}=\xi_{k x_{i}} \partial_{\xi_{k}}, \\
& \operatorname{div}_{u} \mathbb{T}_{u}\left(u, p_{\sigma}\right)=\nabla_{u} \mathbb{T}_{u}\left(u, p_{\sigma}\right) .
\end{aligned}
$$

By (3.4), (3.5) we have respectively

$$
p_{\sigma}=p_{1} \eta_{\sigma}+p_{2} \gamma_{0}
$$

and

$$
p_{\sigma}=p_{3} \bar{\eta}_{\Omega_{t}}+p_{4} \gamma
$$

where

$$
\begin{gathered}
\eta_{\sigma}=\eta-\varrho_{e}, \quad \gamma_{0}=\Gamma-\theta_{e}, \quad \bar{\eta}_{\Omega_{t}}=\eta-\varrho_{\Omega_{t}}, \quad \gamma=\Gamma-\theta_{\Omega_{t}} \\
p_{1}=p_{1}(\eta, \Gamma), \quad p_{2}=p_{2}(\eta, \Gamma), \quad p_{3}=p_{3}\left(\varrho_{\Omega_{t}}, \eta, \Gamma\right) \\
p_{4}=p_{4}\left(\varrho_{\Omega_{t}}, \theta_{\Omega_{t}}, \Gamma\right), \quad p_{i}>0 \quad(i=1,2,3) .
\end{gathered}
$$

Let us introduce a partition of unity $\left(\left\{\widetilde{\Omega}_{i}\right\},\left\{\zeta_{i}\right\}\right), \Omega=\bigcup_{i} \widetilde{\Omega}_{i}$. Let $\widetilde{\Omega}$ be one of the $\widetilde{\Omega}_{i}$ 's and $\zeta(\xi)=\zeta_{i}(\xi)$ be the corresponding function. If $\widetilde{\Omega}$ is an interior subdomain then let $\widetilde{\omega}$ be a set such that $\widetilde{\omega} \subset \widetilde{\Omega}$ and $\zeta(\xi)=1$ for $\xi \in \widetilde{\omega}$. Otherwise we assume that $\overline{\widetilde{\Omega}} \cap S \neq \emptyset, \overline{\widetilde{\omega}} \cap S \neq \emptyset, \overline{\widetilde{\omega}} \subset \overline{\widetilde{\Omega}}$. Take any $\beta \in \widetilde{\widetilde{\omega}} \cap S \subset \overline{\widetilde{\Omega}} \cap S=\overline{\widetilde{S}}$ and introduce local coordinates $\{y\}$ associated with $\{\xi\}$ by

$$
y_{k}=\alpha_{k l}\left(\xi_{l}-\beta_{l}\right), \quad \alpha_{3 k}=n_{k}(\beta), \quad k=1,2,3,
$$


where $\alpha_{k l}$ is a constant orthogonal matrix such that $\widetilde{S}$ is determined by the equation $y_{3}=F\left(y_{1}, y_{2}\right), F \in W_{2}^{4-1 / 2}$ and

$$
\widetilde{\Omega}=\left\{y:\left|y_{i}\right|<d, i=1,2, F\left(y^{\prime}\right)<y_{3}<F\left(y^{\prime}\right)+d, y^{\prime}=\left(y_{1}, y_{2}\right)\right\} .
$$

Next, we introduce functions $u^{\prime}, \eta^{\prime}, \Gamma^{\prime}, \gamma_{0}^{\prime}, \gamma^{\prime}, \Gamma_{1}^{\prime}$ by

$$
\begin{aligned}
u_{i}^{\prime}(y) & =\left.\alpha_{i j} u_{j}(\xi)\right|_{\xi=\xi(y)}, & & \eta^{\prime}(y)=\left.\eta(\xi)\right|_{\xi=\xi(y)}, \\
\Gamma^{\prime}(y) & =\left.\Gamma(\xi)\right|_{\xi=\xi(y)}, & & \gamma_{0}^{\prime}(y)=\left.\gamma_{0}(\xi)\right|_{\xi=\xi(y)}, \\
\gamma^{\prime}(y) & =\left.\gamma(\xi)\right|_{\xi=\xi(y)}, & & \Gamma_{1}^{\prime}(y)=\left.\Gamma_{1}(\xi)\right|_{\xi=\xi(y)},
\end{aligned}
$$

where $\xi=\xi(y)$ is the inverse transformation to (3.40). Further, we introduce new variables by

$$
z_{i}=y_{i} \quad(i=1,2), \quad z_{3}=y_{3}-\widetilde{F}(y), \quad y \in \widetilde{\Omega},
$$

which will be denoted by $z=\Phi(y)$ (where $\widetilde{F}$ is an extension of $F$ with $\left.\widetilde{F} \in W_{2}^{4}\right)$.

Let $\widehat{\Omega}=\Phi(\widetilde{\Omega})=\left\{z:\left|z_{i}\right|<d, i=1,2,0<z_{3}<d\right\}$ and $\widehat{S}=\Phi(\widetilde{S})$. Define

$$
\begin{array}{ll}
\widehat{u}(z)=\left.u^{\prime}(y)\right|_{y=\Phi^{-1}(z)}, & \widehat{\eta}(z)=\left.\eta^{\prime}(y)\right|_{y=\Phi^{-1}(z)}, \\
\widehat{\Gamma}(z)=\left.\Gamma^{\prime}(y)\right|_{y=\Phi^{-1}(z)}, & \widehat{\gamma}_{0}(z)=\left.\gamma_{0}^{\prime}(y)\right|_{y=\Phi^{-1}(z)}, \\
\widehat{\gamma}(z)=\left.\gamma^{\prime}(y)\right|_{y=\Phi^{-1}(z)}, & \widehat{\Gamma}_{1}(z)=\left.\Gamma_{1}^{\prime}(y)\right|_{y=\Phi^{-1}(z)} .
\end{array}
$$

Set $\hat{\nabla}_{k}=\left.\xi_{l x_{k}}(\xi) z_{i \xi_{l}} \nabla_{z_{i}}\right|_{\xi=\chi^{-1}(z)}$, where $\chi(\xi)=\Phi(\psi(\xi))$ and $y=\psi(\xi)$ is described by (3.40). We also introduce the following notation:

$$
\begin{aligned}
\widetilde{u}(\xi) & =u(\xi) \zeta(\xi), & & \widetilde{\eta}(\xi)=\eta(\xi) \zeta(\xi), \\
\widetilde{\Gamma}(\xi) & =\Gamma(\xi) \zeta(\xi), & \widetilde{\gamma}_{0}(\xi) & =\gamma_{0}(\xi) \zeta(\xi), \\
\widetilde{\gamma}(\xi) & =\gamma(\xi) \zeta(\xi), & & \widetilde{\Gamma}_{1}(\xi)=\Gamma_{1}(\xi) \zeta(\xi)
\end{aligned}
$$

for $\xi \in \widetilde{\Omega}, \widetilde{\Omega} \cap S=\emptyset$, and

$$
\begin{array}{rlrl}
\widetilde{u}(z) & =\widehat{u}(z) \widehat{\zeta}(z), & & \widetilde{\eta}(z)=\widehat{\eta}(z) \widehat{\zeta}(z), \\
\widetilde{\Gamma}(z)=\widehat{\Gamma}(z) \widehat{\zeta}(z), & \widetilde{\gamma}_{0}(z)=\widehat{\gamma}_{0}(z) \widehat{\zeta}(z), \\
\widetilde{\gamma}(z)=\widehat{\gamma}(z) \widehat{\zeta}(z), & \widetilde{\Gamma}_{1}(z)=\widehat{\Gamma}_{1}(z) \widehat{\zeta}(z)
\end{array}
$$

for $z \in \widehat{\Omega}=\Phi(\widetilde{\Omega}), \overline{\widetilde{\Omega}} \cap S \neq \emptyset$, where $\widehat{\zeta}(z)=\left.\zeta(\xi)\right|_{\xi=\chi^{-1}(z)}$.

Using the above notation and (3.2) we can rewrite problem (3.36) in the following form in an interior subdomain: 


$$
\begin{aligned}
& \eta \widetilde{u}_{i t}-\nabla_{u_{j}} T_{u}^{i j}\left(\widetilde{u}, \widetilde{p}_{\sigma}\right)=\eta \widetilde{g}_{i}-\nabla_{u_{j}} B_{u}^{i j}(u, \zeta)-T_{u}^{i j}\left(u, p_{\sigma}\right) \nabla_{u_{j}} \zeta \\
& \equiv \eta \widetilde{g}_{i}+k_{1}, \quad i=1,2,3, \\
& \widetilde{\eta}_{\sigma t}+\eta \nabla_{u} \cdot \widetilde{u}=\eta u \cdot \nabla_{u} \zeta \equiv k_{2}, \\
& \eta c_{v}(\eta, \Gamma) \widetilde{\gamma}_{t}-\kappa \nabla_{u}^{2} \widetilde{\gamma}+\Gamma p_{\Gamma}(\eta, \Gamma) \nabla_{u} \cdot \widetilde{u} \\
& =\eta \widetilde{k}+\left[\frac{1}{2} \mu \sum_{i, j=1}^{3}\left(\xi_{k x_{i}} \partial_{\xi_{k}} u_{j}+\xi_{k x_{j}} \partial_{\xi_{k}} u_{i}\right)^{2}+(\nu-\mu)\left(\nabla_{u} \cdot u\right)^{2}\right] \zeta \\
& +\Gamma p_{\Gamma}(\eta, \Gamma) u \cdot \nabla_{u} \zeta-\kappa\left(\nabla_{u}^{2} \zeta \gamma+2 \nabla_{u} \zeta \cdot \nabla_{u} \gamma\right) \\
& -\eta c_{v}(\eta, \Gamma) \zeta \partial_{t} \theta_{\Omega_{t}} \equiv \eta \widetilde{k}+k_{3},
\end{aligned}
$$

where $p_{\sigma}=p_{\sigma} \zeta$ and

$\mathbb{B}_{u}(u, \zeta)=\left\{B_{u}^{i j}(u, \zeta)\right\}=\left\{\mu\left(u_{i} \nabla_{u_{j}} \zeta+u_{j} \nabla_{u_{i}} \zeta\right)+(\nu-\mu) \partial_{i j} u \cdot \nabla_{u} \zeta\right\}$.

In boundary subdomains we have

$$
\begin{aligned}
& \widehat{\eta} \widetilde{u}_{i t}-\widehat{\nabla}_{j} \widehat{T}^{i j}\left(\widetilde{u}, \widetilde{p}_{\sigma}\right)=\widehat{\eta} \widetilde{g}_{i}-\widehat{\nabla}_{j} \widehat{B}^{i j}(\widehat{u}, \widehat{\zeta})-\widehat{T}^{i j}\left(\widehat{u}, p_{\sigma}\right) \widehat{\nabla}_{j} \widehat{\zeta} \\
& \equiv \widehat{\eta} \widetilde{g}_{i}+k_{4}^{i}, \\
& \widetilde{\eta}_{\sigma t}+\widehat{\eta} \widehat{\nabla} \cdot \widetilde{u}=\widehat{\eta} \widehat{u} \cdot \widehat{\nabla} \widehat{\zeta} \equiv k_{5}, \\
& \widehat{\eta} c_{v}(\widehat{\eta}, \widehat{\Gamma}) \widetilde{\gamma}_{t}-\kappa \widehat{\nabla}^{2} \widetilde{\gamma}+\widehat{\Gamma} p_{\Gamma}(\widehat{\eta}, \widehat{\Gamma}) \widehat{\nabla} \cdot \widetilde{u} \\
& =\widehat{\eta} \widetilde{k}+\left[\frac{1}{2} \mu \sum_{i, j=1}^{3}\left(\widehat{\nabla}_{i} \widehat{u}_{j}+\widehat{\nabla}_{j} \widehat{u}_{i}\right)^{2}+(\nu-\mu)(\widehat{\nabla} \cdot \widehat{u})^{2}\right] \widehat{\zeta} \\
& +\widehat{\Gamma} p_{\Gamma}(\widehat{\eta}, \widehat{\Gamma}) \widehat{u} \cdot \widehat{\nabla} \widehat{\zeta}-\kappa\left(\widehat{\nabla}^{2} \widehat{\zeta} \cdot \widehat{\gamma}+2 \widehat{\nabla} \widehat{\zeta} \cdot \widehat{\nabla} \widehat{\gamma}\right) \\
& -\widehat{\eta} c_{v}(\widehat{\eta}, \widehat{\Gamma}) \partial_{t} \theta_{\Omega_{t}} \widehat{\zeta} \equiv \widehat{\eta} \widetilde{k}+k_{6}, \\
& \widehat{\mathbb{T}}\left(\widetilde{u}, \widetilde{p}_{\sigma}\right) \widehat{n}=k_{7}, \\
& \widehat{n} \cdot \widehat{\nabla} \widetilde{\gamma}=\widetilde{\Gamma}_{1}+k_{8},
\end{aligned}
$$

where $k_{7}^{i}=\widehat{B}^{i j}(\widehat{u}, \widehat{\zeta}) \widehat{n}_{j}, k_{8}=\widehat{n} \cdot \widehat{\nabla} \widehat{\zeta} \widehat{\gamma}, \widehat{\nabla}=\left(\widehat{\nabla}_{j}\right)_{j=1,2,3}$, and $\widehat{T}$ and $\widehat{B}$ indicate that the operator $\nabla_{u}$ is replaced by $\hat{\nabla}$.

In the considerations below we denote $z_{1}, z_{2}$ by $\tau$ and $z_{3}$ by $n$.

LEMMA 3.4. Let $v, \varrho, \vartheta_{0}$ be a sufficiently smooth solution of problem (3.3). Then

$$
\begin{aligned}
& \frac{1}{2} \frac{d}{d t} \int_{\Omega_{t}}\left(\varrho v_{x}^{2}+\frac{p_{\sigma \varrho}}{\varrho} \varrho_{\sigma x}^{2}+\frac{\varrho c_{v}}{\theta} \vartheta_{0 x}^{2}\right) d x \\
& \quad+c_{0}\left(\left\|v_{x}\right\|_{1, \Omega_{t}}^{2}+\left\|\bar{\varrho}_{\Omega_{t}}\right\|_{0, \Omega_{t}}^{2}+\left\|\varrho_{\sigma x}\right\|_{0, \Omega_{t}}^{2}+\left\|\varrho_{\sigma t}\right\|_{0, \Omega_{t}}^{2}+\left\|\vartheta_{0 x}\right\|_{1, \Omega_{t}}^{2}\right)
\end{aligned}
$$




$$
\begin{aligned}
\leq & \varepsilon\left(\left\|v_{x t}\right\|_{0, \Omega_{t}}^{2}+\left\|\vartheta_{0 x t}\right\|_{0, \Omega_{t}}^{2}\right) \\
& +C_{1}\left(|v|_{1,0, \Omega_{t}}^{2}+\left\|\bar{\varrho}_{\Omega_{t}}\right\|_{0, \Omega_{t}}^{2}+\|\vartheta\|_{0, \Omega_{t}}^{2}+\left\|\vartheta_{0 x}\right\|_{0, \Omega_{t}}^{2}\right. \\
& \left.+\left\|\vartheta_{0 t}\right\|_{0, \Omega_{t}}^{2}+\|f\|_{1, \Omega_{t}}^{2}+\|r\|_{1, \Omega_{t}}^{2}+\left\|\theta_{1}\right\|_{2, \Omega_{t}}^{2}\right) \\
& +C_{2}\left(X_{2}+\int_{0}^{t}\|v\|_{3, \Omega_{t^{\prime}}}^{2} d t^{\prime}\right) Y_{2},
\end{aligned}
$$

where

$$
\begin{gathered}
v_{x}^{2}=\sum_{i, j=1}^{3} v_{i x_{j}}^{2}, \quad \varrho_{\sigma x}^{2}=\sum_{i=1}^{3} \varrho_{\sigma x_{i}}^{2}, \quad \vartheta_{0 x}^{2}=\sum_{i=1}^{3} \vartheta_{0 x_{i}}^{2}, \\
X_{2}=|v|_{2,1, \Omega_{t}}^{2}+\left|\varrho_{\sigma}\right|_{2,1, \Omega_{t}}^{2}+\left|\vartheta_{0}\right|_{2,1, \Omega_{t}}^{2}+\left\|\bar{\varrho}_{\Omega_{t}}\right\|_{0, \Omega_{t}}^{2}, \\
Y_{2}=X_{2}+\|v\|_{3, \Omega_{t}}^{2}+\left\|\vartheta_{0 x}\right\|_{2, \Omega_{t}}^{2}+\|\vartheta\|_{0, \Omega_{t}}^{2}+\left\|\varrho_{\Omega_{t}}\right\|_{0, \Omega_{t}}^{2} .
\end{gathered}
$$

P r o of. First we obtain the estimate in interior subdomains. Differentiating $(3.46)_{1}$ with respect to $\xi$, multiplying the result by $\widetilde{u}_{\xi} A$ (where $A$ is the Jacobian of the transformation $x=x(\xi))$ and integrating over $\widetilde{\Omega}$ we get

$$
\begin{aligned}
& \frac{1}{2} \frac{d}{d t} \int_{\tilde{\Omega}} \eta \widetilde{u}_{\xi}^{2} A d \xi+\frac{1}{2} \mu \int_{\tilde{\Omega}}\left(\nabla_{u_{i}} \widetilde{u}_{j \xi}+\nabla_{u_{j}} \widetilde{u}_{i \xi}\right)^{2} A d \xi \\
& \quad+(\nu-\mu)\left\|\nabla_{u} \cdot \widetilde{u}_{\xi}\right\|_{0, \tilde{\Omega}}^{2}-\int_{\tilde{\Omega}} \widetilde{p}_{\sigma \xi} \cdot\left(\nabla_{u} \cdot \widetilde{u}_{\xi}\right) A d \xi \\
& \leq \varepsilon\left(\left\|u_{\xi \xi}\right\|_{0, \tilde{\Omega}}^{2}+\left\|\eta_{\sigma \xi}\right\|_{0, \tilde{\Omega}}^{2}+\left\|\gamma_{0 \xi}\right\|_{0, \tilde{\Omega}}^{2}\right) \\
&+C_{1}\left(\|u\|_{1, \tilde{\Omega}}^{2}+\left\|\gamma_{0 \xi}\right\|_{0, \tilde{\Omega}}^{2}+\left\|\bar{\eta}_{\Omega_{t}}\right\|_{0, \tilde{\Omega}}^{2}+\|\gamma\|_{0, \tilde{\Omega}}^{2}+\|\widetilde{g}\|_{0, \tilde{\Omega}}^{2}\right) \\
&+C_{2}\left(X_{2}(\widetilde{\Omega})+\int_{0}^{t}\|u\|_{3, \tilde{\Omega}}^{2} d t^{\prime}\right) Y_{2}(\widetilde{\Omega}),
\end{aligned}
$$

where $\|h\|_{0, \tilde{\Omega}}=\left(\int_{\tilde{\Omega}}|h|^{2} A d \xi\right)^{1 / 2}, \widetilde{u}_{\xi}^{2}=\sum_{i=1}^{3} \widetilde{u}_{i \xi}^{2}$ and

$$
\begin{aligned}
& X_{2}(\widetilde{\Omega})=|u|_{2,1, \tilde{\Omega}}^{2}+\left|\eta_{\sigma}\right|_{2,1, \tilde{\Omega}}^{2}+\left|\gamma_{0}\right|_{2,1, \tilde{\Omega}}^{2}+\left\|\bar{\eta}_{\Omega_{t}}\right\|_{0, \tilde{\Omega}}^{2}, \\
& Y_{2}(\widetilde{\Omega})=X_{2}(\widetilde{\Omega})+\|u\|_{3, \tilde{\Omega}}^{2}+\|\gamma\|_{3, \tilde{\Omega}}^{2}+\left\|\bar{\eta}_{\Omega_{t}}\right\|_{0, \tilde{\Omega}}^{2} .
\end{aligned}
$$

Next, we have

$$
\begin{aligned}
-\int_{\tilde{\Omega}} \widetilde{p}_{\sigma \xi}\left(\nabla_{u} \cdot \widetilde{u}_{\xi}\right) A d \xi= & -\int_{\tilde{\Omega}} p_{\sigma \xi} \widetilde{\gamma}_{\xi}\left(\nabla_{u} \cdot \widetilde{u}_{\xi}\right) A d \xi \\
& -\int_{\tilde{\Omega}} p_{\sigma \xi} \widetilde{\bar{\eta}}_{\Omega_{t} \xi}\left(\nabla_{u} \cdot \widetilde{u}_{\xi}\right) A d \xi+I_{5},
\end{aligned}
$$


where

$$
\left|I_{5}\right| \leq \varepsilon\left\|\widetilde{u}_{\xi \xi}\right\|_{0, \tilde{\Omega}}^{2}+C_{1}\left(\|\gamma\|_{0, \tilde{\Omega}}^{2}+\left\|\bar{\eta}_{\Omega_{t}}\right\|_{0, \tilde{\Omega}}^{2}\right) .
$$

In order to consider $-\int_{\tilde{\Omega}} p_{\sigma \xi} \widetilde{\bar{\eta}}_{\Omega_{t} \xi}\left(\nabla_{u} \cdot \widetilde{u}_{\xi}\right) A d \xi$ we rewrite equation (3.21) in the Lagrangian coordinates to obtain

$$
\partial_{t} \widetilde{\bar{\eta}}_{\Omega_{t}}+\eta \nabla_{u} \cdot \widetilde{u}=\eta u \cdot \nabla_{u} \zeta-\zeta \partial_{t} \varrho_{\Omega_{t}} .
$$

Differentiating (3.52) with respect to $\xi$ yields

$$
\begin{aligned}
\nabla_{u} \cdot \widetilde{u}_{\xi}= & -\frac{\partial_{t} \widetilde{\bar{\eta}}_{\Omega_{t} \xi}}{\eta}+\frac{\eta_{\sigma \xi} \nabla_{u} \cdot \widetilde{u}}{\eta}-\xi_{x}^{\prime} \int_{0}^{t} u_{\xi \xi} d t^{\prime} \widetilde{u}_{\xi}+\frac{\eta_{\sigma \xi}}{\eta} u \cdot \nabla_{u} \zeta \\
& +u_{\xi} \nabla_{u} \zeta+u \xi_{x}^{\prime} \int_{0}^{t} u_{\xi \xi} d t^{\prime} \zeta_{\xi}+u \nabla_{u} \zeta_{\xi}-\frac{\zeta_{\xi}}{\eta} \partial_{t} \varrho_{\Omega_{t}} .
\end{aligned}
$$

Hence

$$
\begin{aligned}
(3.53)-\int_{\tilde{\Omega}} p_{\sigma \xi} \widetilde{\bar{\eta}}_{\Omega_{t} \xi}\left(\nabla_{u} \cdot \widetilde{u}_{\xi}\right) A d \xi= & \frac{1}{2} \frac{d}{d t} \int_{\tilde{\Omega}} \frac{p_{\sigma \xi}}{\eta} \widetilde{\bar{\eta}}_{\Omega_{t} \xi}^{2} A d \xi \\
& +\int_{\tilde{\Omega}} p_{\sigma \xi} \widetilde{\bar{\eta}}_{\Omega_{t} \xi} \frac{\zeta_{\xi}}{\eta} \partial_{t} \varrho_{\Omega_{t}} d \xi+I_{6}
\end{aligned}
$$

where

$$
\begin{aligned}
\left|I_{6}\right| \leq & \varepsilon\left\|\eta_{\sigma \xi}\right\|_{0, \tilde{\Omega}}^{2}+C_{1}\left(\left\|\bar{\eta}_{\Omega_{t}}\right\|_{0, \tilde{\Omega}}^{2}+\|u\|_{1, \tilde{\Omega}}^{2}\right) \\
& +C_{2}\left[\left\|\eta_{\sigma}\right\|_{2, \tilde{\Omega}}^{2}\|u\|_{2, \tilde{\Omega}}^{2}+\|u\|_{2, \tilde{\Omega}}^{2}\left\|\int_{0}^{t} u d t^{\prime}\right\|_{3, \tilde{\Omega}}^{2}\right. \\
& \left.+\left\|\bar{\eta}_{\Omega_{t}}\right\|_{2, \tilde{\Omega}}^{2}\left(\left|\eta_{\sigma}\right|_{2,1, \tilde{\Omega}}^{2}+\left|\gamma_{0}\right|_{2,1, \tilde{\Omega}}^{2}+\|u\|_{2, \tilde{\Omega}}^{2}\right)\right]
\end{aligned}
$$

and by (3.22) and (3.23),

$$
\begin{aligned}
\int_{\tilde{\Omega}}\left|p_{\sigma \eta} \widetilde{\bar{\eta}}_{\Omega_{t} \xi} \frac{\zeta_{\xi}}{\eta} \partial_{t} \varrho_{\Omega_{t}}\right| d \xi & \\
& \leq \varepsilon\left\|\eta_{\sigma \xi}\right\|_{0, \tilde{\Omega}}^{2}+C_{1}\left(\left\|\bar{\eta}_{\Omega_{t}}\right\|_{0, \tilde{\Omega}}^{2}+\left\|\vartheta_{0 t}\right\|_{0, \tilde{\Omega}}^{2}+\|v\|_{1, \tilde{\Omega}}^{2}\right) .
\end{aligned}
$$

Next, dividing $(3.46)_{3}$ by $\Gamma$, differentiating the result with respect to $\xi$, multiplying by $\widetilde{\gamma}_{\xi} A$ and integrating over $\widetilde{\Omega}$ yields

(3.56) $\quad \frac{1}{2} \frac{d}{d t} \int_{\tilde{\Omega}} \frac{\eta c_{v}}{\Gamma} \widetilde{\gamma}_{\xi}^{2} A d \xi+\int_{\tilde{\Omega}} p_{\sigma \Gamma} \nabla_{u} \cdot \widetilde{u}_{\xi} \widetilde{\gamma}_{\xi} A d \xi+\int_{\tilde{\Omega}} \frac{\kappa}{\Gamma}\left|\nabla_{u} \widetilde{\gamma}_{\xi}\right|^{2} A d \xi$ 


$$
\begin{aligned}
\leq & \varepsilon\left(\left\|\eta_{\sigma \xi}\right\|_{0, \tilde{\Omega}}^{2}+\left\|\widetilde{\gamma}_{\xi \xi}\right\|_{0, \tilde{\Omega}}^{2}+\left\|\widetilde{u}_{\xi \xi}\right\|_{0, \tilde{\Omega}}^{2}\right) \\
& +C_{1}\left(\left\|\gamma_{0 \xi}\right\|_{0, \tilde{\Omega}}^{2}+\|\gamma\|_{0, \tilde{\Omega}}^{2}+\left\|\vartheta_{0 t}\right\|_{0, \Omega_{t}}^{2}+\|u\|_{1, \tilde{\Omega}}^{2}+\|v\|_{1, \Omega_{t}}^{2}+\|\widetilde{k}\|_{0, \tilde{\Omega}}^{2}\right) \\
& +C_{2}\left[\left(X_{2}(\widetilde{\Omega})+\int_{0}^{t}\|u\|_{3, \tilde{\Omega}}^{2} d t^{\prime}\right) Y_{2}(\widetilde{\Omega})+\|\gamma\|_{2, \tilde{\Omega}}^{2}\left(\left\|\vartheta_{0 t}\right\|_{0, \Omega_{t}}^{2}+\|v\|_{1, \Omega_{t}}^{2}\right)\right] .
\end{aligned}
$$

Consider now the Stokes problem

$$
\begin{aligned}
& \mu \nabla_{u}^{2} \widetilde{u}-\nu \nabla_{u} \nabla_{u} \cdot \widetilde{u}+p_{\sigma \eta} \nabla_{u} \widetilde{\bar{\eta}}_{\Omega_{t}} \\
& \quad=\eta \widetilde{g}-\eta \widetilde{u}_{t}-\widetilde{p}_{\sigma \Gamma} \nabla_{u} \gamma_{0}-p_{\sigma \eta} \nabla_{u} \zeta \bar{\eta}_{\Omega_{t}}+k_{1}, \\
& \nabla_{u} \cdot \widetilde{u}=\nabla_{u} \cdot \widetilde{u}, \\
& \left.\widetilde{u}\right|_{\partial \tilde{\Omega}}=0 .
\end{aligned}
$$

For $\widetilde{u}$ and $\widetilde{\bar{\eta}}_{\Omega_{t}}$ satisfying (3.57) we have

$$
\begin{aligned}
& \|\widetilde{u}\|_{2, \tilde{\Omega}}^{2}+\left\|\widetilde{\bar{\eta}}_{\Omega_{t}}\right\|_{1, \tilde{\Omega}}^{2} \\
\leq & C_{1}\left(|u|_{1,0, \tilde{\Omega}}^{2}+\|\gamma\|_{0, \tilde{\Omega}}^{2}+\left\|\gamma_{0 \xi}\right\|_{0, \tilde{\Omega}}^{2}+\left\|\bar{\eta}_{\Omega_{t}}\right\|_{0, \tilde{\Omega}}^{2}+\|\widetilde{g}\|_{0, \tilde{\Omega}}^{2}\right) \\
& +C_{2}\left[\left(\|u\|_{2, \tilde{\Omega}}^{2}+\left\|\gamma_{0}\right\|_{2, \tilde{\Omega}}^{2}+\left\|\eta_{\sigma}\right\|_{1, \tilde{\Omega}}^{2}\right)\left\|\int_{0}^{t} u d t^{\prime}\right\|_{3, \tilde{\Omega}}^{2}\right]+c\left\|\nabla_{u} \cdot \widetilde{u}\right\|_{1, \tilde{\Omega}}^{2} .
\end{aligned}
$$

Summing up inequalities (3.49)-(3.51), (3.53)-(3.55), (3.56), (3.58) and using Lemma 5.1 of $[21]$ in the case $G=\bar{\Omega}, v=\widetilde{u}_{\xi}$ we obtain

$$
\begin{aligned}
& \frac{1}{2} \frac{d}{d t} \int_{\tilde{\Omega}}\left(\eta \widetilde{u}_{\xi}^{2}+\frac{p_{\sigma \eta}}{\eta} \widetilde{\eta}_{\Omega_{t} \xi}^{2}+\frac{\eta c_{v}}{\Gamma} \widetilde{\gamma}_{\xi}^{2}\right) A d \xi \\
& +\frac{1}{2} \mu\left\|\widetilde{u}_{\xi}\right\|_{1, \tilde{\Omega}}^{2}+\frac{\kappa}{\theta^{*}}\left\|\widetilde{\gamma}_{\xi \xi}\right\|_{0, \tilde{\Omega}}^{2}+\left\|\widetilde{\bar{\eta}}_{\Omega_{t}}\right\|_{1, \tilde{\Omega}}^{2} \\
\leq & \varepsilon\left(\left\|\widetilde{u}_{\xi \xi}\right\|_{0, \tilde{\Omega}}^{2}+\left\|\eta_{\sigma \xi}\right\|_{0, \tilde{\Omega}}^{2}+\left\|\widetilde{\gamma}_{\xi \xi}\right\|_{0, \tilde{\Omega}}^{2}\right) \\
& +C_{1}\left(|u|_{1,0, \tilde{\Omega}}^{2}+\|v\|_{1, \Omega_{t}}^{2}+\left\|\gamma_{0 \xi}\right\|_{0, \tilde{\Omega}}^{2}+\|\gamma\|_{0, \tilde{\Omega}}^{2}\right. \\
& \left.+\left\|\vartheta_{0 t}\right\|_{0, \Omega_{t}}^{2}+\left\|\bar{\eta}_{\Omega_{t}}\right\|_{0, \tilde{\Omega}}^{2}+\|\widetilde{g}\|_{0, \tilde{\Omega}}^{2}+\|\widetilde{k}\|_{0, \tilde{\Omega}}^{2}\right) \\
& +C_{2}\left[\left(X_{2}(\widetilde{\Omega})+\int_{0}^{t}\|u\|_{3, \tilde{\Omega}}^{2} d t^{\prime}\right) Y_{2}(\widetilde{\Omega})+\|\gamma\|_{2, \tilde{\Omega}}^{2}\left(\left\|\vartheta_{0 t}\right\|_{0, \Omega_{t}}^{2}+\|v\|_{1, \Omega_{t}}^{2}\right)\right] .
\end{aligned}
$$

Now we consider subdomains near the boundary. Differentiate $(3.47)_{1}$ with respect to $\tau$, multiply the result by $\widetilde{u}_{\tau} J$ and integrate over $\widehat{\Omega}$ ( $J$ is the Jacobian of $x=x(z))$. Next, divide (3.47) $)_{3}$ by $\widehat{\Gamma}$, differentiate the result with respect to $\tau$, multiply by $\widetilde{\gamma}_{\tau} J$ and integrate over $\widehat{\Omega}$. Hence using Lemma 5.1 of [21] and equation $(3.47)_{2}$ we get 


$$
\begin{aligned}
\text { (3.60) } & \frac{1}{2} \frac{d}{d t} \int_{\hat{\Omega}}\left(\widehat{\eta} \widetilde{u}_{\tau}^{2}+\frac{p_{\sigma \hat{\eta}}}{\widehat{\eta}} \widetilde{\bar{\eta}}_{\Omega_{t} \tau}^{2}+\frac{\widehat{\eta} c_{v}}{\widehat{\Gamma}} \widetilde{\gamma}_{\tau}^{2}\right) J d z+c_{0}\left(\left\|\widetilde{u}_{\tau}\right\|_{1, \hat{\Omega}}^{2}+\left\|\widetilde{\gamma}_{\tau z}\right\|_{0, \hat{\Omega}}^{2}\right) \\
& -\int_{\hat{S}}\left(\widehat{\mathbb{T}}\left(\widetilde{u}, \widetilde{p}_{\sigma}\right) \widehat{n}\right)_{, \tau} \widetilde{u}_{\tau} J d z^{\prime}-\kappa \int_{\hat{S}}\left(\widehat{n} \cdot \widehat{\Gamma}^{-1} \widehat{\nabla} \widetilde{\gamma}\right)_{, \tau} \widetilde{\gamma}_{\tau} J d z^{\prime} \\
\leq & \varepsilon\left(\left\|\widetilde{u}_{z z}\right\|_{0, \hat{\Omega}}^{2}+\left\|\widehat{\eta}_{\sigma z}\right\|_{0, \hat{\Omega}}^{2}+\left\|\widehat{\gamma}_{0 z z}\right\|_{0, \hat{\Omega}}^{2}\right) \\
& +C_{1}\left(|\widehat{u}|_{1,0, \hat{\Omega}}^{2}+\|v\|_{1, \Omega_{t}}^{2}+\left\|\widehat{\gamma}_{0 \tau}\right\|_{0, \hat{\Omega}}^{2}+\|\widehat{\gamma}\|_{0, \hat{\Omega}}^{2}\right. \\
& \left.+\left\|\vartheta_{0 t}\right\|_{0, \Omega_{t}}^{2}+\left\|\widehat{\bar{\eta}}_{\Omega_{t}}\right\|_{0, \hat{\Omega}}^{2}+\|\widetilde{g}\|_{1, \hat{\Omega}}^{2}+\|\widetilde{k}\|_{1, \hat{\Omega}}^{2}\right) \\
& +C_{2}\left[\left(X_{2}(\widehat{\Omega})+\int_{0}^{t}\|\widehat{u}\|_{3, \hat{\Omega}}^{2} d t^{\prime}\right) Y_{2}(\widehat{\Omega})+\|\widehat{\gamma}\|_{2, \hat{\Omega}}^{2}\left(\left\|\vartheta_{0 t}\right\|_{0, \Omega_{t}}^{2}+\|v\|_{1, \Omega_{t}}^{2}\right)\right],
\end{aligned}
$$

where $X_{2}(\widehat{\Omega})$ and $Y_{2}(\widehat{\Omega})$ are defined analogously to $X_{2}(\widetilde{\Omega})$ and $Y_{2}(\widetilde{\Omega})$.

Using the boundary conditions $(3.47)_{4}$ and $(3.47)_{5}$ we have

$$
\begin{aligned}
& -\int_{\hat{S}}\left(\widehat{\mathbb{T}}\left(\widetilde{u}, \widetilde{p}_{\sigma}\right) \widehat{n}\right)_{, \tau} \widetilde{u}_{\tau} J d z^{\prime} \\
& \quad \leq \varepsilon\left\|\widehat{u}_{z z}\right\|_{0, \hat{\Omega}}^{2}+C_{1}\|\widehat{u}\|_{1, \hat{\Omega}}^{2}+C_{2}\|\widehat{u}\|_{2, \hat{\Omega}}^{2}\left\|\int_{0}^{t} \widehat{u} d t^{\prime}\right\|_{3, \hat{\Omega}}^{2}
\end{aligned}
$$

and

$$
\begin{aligned}
-\kappa \int_{\hat{S}}\left(\widehat{n} \cdot \widehat{\Gamma}^{-1} \widehat{\nabla} \widehat{\gamma}\right)_{, \tau} \widetilde{\gamma}_{\tau} J d z^{\prime} \\
\leq \varepsilon\left\|\widehat{\gamma}_{0 z z}\right\|_{0, \hat{\Omega}}^{2}+C_{1}\left(\|\widehat{\gamma}\|_{0, \hat{\Omega}}^{2}+\left\|\widehat{\gamma}_{0 z}\right\|_{0, \hat{\Omega}}^{2}+\left\|\widetilde{\Gamma}_{1}\right\|_{2, \hat{\Omega}}^{2}\right) \\
\quad+C_{2}\|\widehat{\gamma}\|_{2, \hat{\Omega}}^{2}\left(\left\|\widehat{\gamma}_{0}\right\|_{2, \hat{\Omega}}^{2}+\|\widehat{\gamma}\|_{2, \hat{\Omega}}^{2}+\left\|\widehat{\eta}_{\sigma}\right\|_{2, \hat{\Omega}}^{2}+\left\|\int_{0}^{t} \widehat{u} d t^{\prime}\right\|_{3, \hat{\Omega}}^{2}\right) .
\end{aligned}
$$

To obtain (3.61) and (3.62) we have applied the interpolation inequality (see Lemma 2.1).

Writing equation (3.52) in the coordinates $z$ we obtain

$$
\partial_{t} \widetilde{\bar{\eta}}_{\Omega_{t}}+\widehat{\eta} \widehat{\nabla} \cdot \widetilde{u}=\widehat{\eta} \widehat{u} \cdot \widehat{\nabla} \widehat{\zeta}-\widehat{\zeta} \partial_{t} \varrho_{\Omega_{t}} .
$$

Applying now the operator $(\mu+\nu) \nabla_{z_{i}}$ to (3.63), dividing the result by $\widehat{\eta}$, adding to $(3.47)_{1}$ and multiplying both sides of the result by $p_{\sigma \hat{\eta}}$ gives

(3.64) $\quad \frac{(\mu+\nu) p_{\sigma \hat{\eta}}}{\widehat{\eta}} \nabla_{z_{i}} \partial_{t} \widetilde{\bar{\eta}}_{\Omega_{t}}+p_{\sigma \hat{\eta}}^{2} \widehat{\nabla}_{i} \widetilde{\bar{\eta}}_{\Omega_{t}}$

$$
=p_{3} p_{\sigma \hat{\eta}} \widehat{\bar{\eta}}_{\Omega_{t}} \widehat{\nabla}_{i} \widehat{\zeta}-p_{\sigma \hat{\eta}} p_{\sigma \hat{\Gamma}} \widehat{\zeta} \widehat{\nabla}_{i} \widehat{\gamma}_{0}-p_{\sigma \hat{\eta}}^{2} \widehat{\nabla}_{i} \widehat{\zeta} \widehat{\bar{\eta}}_{\Omega_{t}}-p_{3} p_{\sigma \hat{\eta}} \widehat{\nabla_{i}} \widehat{\zeta} \widehat{\gamma}-p_{\sigma \hat{\eta}} \hat{\eta} \widetilde{u}_{i t}
$$




$$
\begin{aligned}
& +p_{\sigma \hat{\eta}} \widehat{\eta} \widetilde{g}_{i}-\frac{(\mu+\nu)}{\widehat{\eta}} p_{\sigma \hat{\eta}} \nabla_{z_{i}} \hat{\eta} \widehat{\nabla} \cdot \widetilde{u}+\frac{(\mu+\nu)}{\widehat{\eta}} p_{\sigma \hat{\eta}} \nabla_{z_{i}}(\widehat{\eta} \widehat{u} \cdot \widehat{\nabla} \widehat{\zeta}) \\
& +\mu p_{\sigma \hat{\eta}}\left(\widehat{\nabla}^{2} \widetilde{u}_{i}-\widehat{\nabla}_{i} \widehat{\nabla} \cdot \widetilde{u}\right)+(\mu+\nu) p_{\sigma \hat{\eta}}\left(\widehat{\nabla}_{i}-\nabla_{z_{i}}\right) \widehat{\nabla} \cdot \widetilde{u} \\
& -\frac{(\mu+\nu)}{\widehat{\eta}} p_{\sigma \hat{\eta}} \nabla_{z_{i}} \widehat{\zeta} \partial_{t} \varrho_{\Omega_{t}}+p_{\sigma \hat{\eta}} k_{4}^{i} .
\end{aligned}
$$

Multiplying the normal component of (3.64) by $\widetilde{\eta}_{\sigma n} J$ and integrating over $\widehat{\Omega}$ implies

$$
\begin{aligned}
& \frac{1}{2} \frac{d}{d t} \int_{\hat{\Omega}} \frac{p_{\sigma \hat{\eta}}}{\widehat{\eta}} \widetilde{\bar{\eta}}_{\Omega_{t} n}^{2} J d z+c_{0}\left\|\widetilde{\bar{\eta}}_{\Omega_{t} n}\right\|_{0, \hat{\Omega}}^{2} \\
& \leq(\varepsilon+c d)\left(\left\|\widetilde{\bar{\eta}}_{\Omega_{t} n}\right\|_{0, \hat{\Omega}}^{2}+\left\|\widetilde{u}_{n n}\right\|_{0, \hat{\Omega}}^{2}\right) \\
&+C_{1}\left(\left\|\widehat{\bar{\eta}}_{\Omega_{t}}\right\|_{0, \hat{\Omega}}^{2}+\left\|\widehat{\gamma}_{0 z}\right\|_{0, \hat{\Omega}}^{2}+\|\widehat{\gamma}\|_{0, \hat{\Omega}}^{2}+\left\|\vartheta_{0 t}\right\|_{0, \Omega_{t}}^{2}\right. \\
&\left.+|\widehat{u}|_{1,0, \hat{\Omega}}^{2}+\|v\|_{1, \Omega_{t}}^{2}+\left\|\widetilde{u}_{z \tau}\right\|_{0, \hat{\Omega}}^{2}+\|\widetilde{g}\|_{0, \hat{\Omega}}^{2}\right) \\
&+C_{2}\left[\left\|\widehat{\eta}_{\sigma}\right\|_{2, \hat{\Omega}}^{2}\|\widehat{u}\|_{2, \hat{\Omega}}^{2}+\left\|\widehat{\bar{\eta}}_{\Omega_{t}}\right\|_{2, \Omega_{t}}^{2}\right. \\
& \times\left(\left\|\widehat{\eta}_{\sigma t}\right\|_{1, \hat{\Omega}}^{2}+\|\widehat{u}\|_{2, \hat{\Omega}}^{2}+\left\|\widehat{\gamma}_{0 t}\right\|_{1, \hat{\Omega}}^{2}+\left\|\int_{0}^{t} \widehat{u} d t^{\prime}\right\|_{3, \hat{\Omega}}^{2}\right) \\
&\left.+\|\widehat{u}\|_{2, \hat{\Omega}}^{2}\left\|\int_{0}^{t} \widehat{u} d t^{\prime}\right\|_{3, \hat{\Omega}}^{2}+\|\widehat{u}\|_{3, \hat{\Omega}}^{2}\left\|\int_{0}^{t} \widehat{u} d t^{\prime}\right\|_{2, \hat{\Omega}}^{2}\right] .
\end{aligned}
$$

Now, write $(3.47)_{1}$ in the form

$$
\widehat{\eta} \widetilde{u}_{i t}-\mu \Delta \widetilde{u}_{i}-\nabla_{z_{i}} \nabla \cdot \widetilde{u}=\widehat{\nabla}_{i} \widetilde{p}_{\sigma}+\widehat{\eta} \widetilde{g}_{i}+k_{4}^{i}-k_{9}^{i},
$$

where

$$
k_{9}^{i}=\left(\mu \Delta \widetilde{u}_{i}+\nu \nabla_{z_{i}} \nabla \cdot \widetilde{u}\right)-\left(\mu \widehat{\nabla}^{2} \widetilde{u}_{i}+\nu \widehat{\nabla}_{i} \widehat{\nabla} \cdot \widetilde{u}\right) .
$$

Multiplying the third component of (3.66) by $\widetilde{u}_{3 n n} J$ and integrating over $\widehat{\Omega}$ yields

$$
\begin{aligned}
& \frac{1}{2} \frac{d}{d t} \int_{\hat{\Omega}} \widehat{\eta}_{3 n}^{2} J d z+c_{0}\left\|\widetilde{u}_{3 n n}\right\|_{0, \hat{\Omega}}^{2} \\
& \leq(\varepsilon+c d)\left\|\widetilde{u}_{n n}\right\|_{0, \hat{\Omega}}^{2}+\varepsilon\left\|\widetilde{u}_{3 n t}\right\|_{0, \hat{\Omega}}^{2} \\
&+C_{1}\left(\left\|\widetilde{u}_{z \tau}\right\|_{0, \hat{\Omega}}^{2}+\|\widehat{u}\|_{1, \hat{\Omega}}^{2}+\left\|\widetilde{u}_{t}\right\|_{0, \hat{\Omega}}^{2}+\left\|\widetilde{\bar{\eta}}_{\Omega_{t} n}\right\|_{0, \hat{\Omega}}^{2}\right. \\
&\left.+\left\|\widehat{\bar{\eta}}_{\Omega_{t}}\right\|_{0, \hat{\Omega}}^{2}+\left\|\widehat{\gamma}_{0 z}\right\|_{0, \hat{\Omega}}^{2}+\|\widehat{\gamma}\|_{0, \hat{\Omega}}^{2}+\|\widetilde{g}\|_{0, \hat{\Omega}}^{2}\right) \\
&+C_{2}\left(\left\|\widehat{\eta}_{\sigma t}\right\|_{1, \hat{\Omega}}^{2}\|\widetilde{u}\|_{2, \hat{\Omega}}^{2}+\|\widetilde{u}\|_{2, \hat{\Omega}}^{4}+\|\widetilde{u}\|_{2, \hat{\Omega}}^{2}\left\|\int_{0}^{t} \widehat{u} d t^{\prime}\right\|_{3, \hat{\Omega}}^{2}\right) .
\end{aligned}
$$


To estimate $\widetilde{u}_{i n n}(i=1,2)$ and $\widetilde{\bar{\eta}}_{\Omega_{t} \tau}$ we rewrite (3.66) as

$$
\begin{aligned}
-\mu \Delta \widetilde{u}_{i}+\nabla_{z_{i}}\left(p_{\sigma \hat{\eta}} \widetilde{\bar{\eta}}_{\Omega_{t}}\right)= & \widehat{\eta} \widetilde{g}_{i}-\widehat{\eta} \widetilde{u}_{i t}+k_{4}^{i}-k_{9}^{i}+\nabla_{z_{i}}\left(p_{\sigma \hat{\eta}} \widetilde{\bar{\eta}}_{\Omega_{t}}\right) \\
& -\widehat{\nabla}_{i} \widetilde{p}_{\sigma}+\nu \nabla_{z_{i}} \operatorname{div} \widetilde{u} \\
\equiv & \widetilde{f}_{i}+\nu \nabla_{z_{i}} \operatorname{div} \widetilde{u}
\end{aligned}
$$

and the boundary condition $(3.47)_{4}$ as

$$
\begin{array}{r}
\frac{\partial \widetilde{u}_{i}}{\partial z_{3}}=-\frac{\partial \widetilde{u}_{3}}{\partial z_{i}}+\left(\frac{\partial \widetilde{u}_{i}}{\partial z_{3}}+\frac{\partial \widetilde{u}_{3}}{\partial z_{i}}-\mu^{-1} \widehat{\tau_{i}} \widehat{T} \widehat{n}\right)+\mu^{-1} k_{7} \cdot \widehat{\tau}_{i} \equiv \widetilde{h}_{i}, \\
i=1,2, \quad z_{3}=0
\end{array}
$$

where we have also used the fact that $\widehat{\tau}_{i} \cdot \widehat{n}=0, i=1,2$. When considering problem (3.68)-(3.69) in $\widehat{\Omega}$ we have to add the boundary conditions

$$
\begin{gathered}
\left.\widetilde{u}_{i}\right|_{\left|z^{\prime}\right|=d}=0,\left.\quad \widetilde{u}_{i}\right|_{z_{3}=d}=0, \quad i=1,2, \\
\left.\widetilde{\bar{\eta}}_{\Omega_{t}}\right|_{\left|z^{\prime}\right|=d}=0,\left.\quad \widetilde{\bar{\eta}}_{\Omega_{t}}\right|_{z_{3}=d}=0 .
\end{gathered}
$$

Multiplying (3.68) by $\widetilde{u}_{i}$, summing over $i=1,2$, integrating over $\widehat{\Omega}$ and using the boundary conditions (3.69) and (3.70) yields

$$
\left\|\widetilde{u}_{z}^{\prime}\right\|_{0, \hat{\Omega}}^{2} \leq \varepsilon\left\|\widetilde{\bar{\eta}}_{\Omega_{t}}\right\|_{0, \hat{\Omega}}^{2}+c\left(\left\|\widetilde{f}^{\prime}\right\|_{0, \hat{\Omega}}^{2}+\left\|\widetilde{h}^{\prime}\right\|_{0, \hat{\Omega}}^{2}\right)+C_{1}\|\operatorname{div} \widetilde{u}\|_{0, \hat{\Omega}}^{2},
$$

where the prime indicates that only two components $(i=1,2)$ are taken into account.

In order to estimate $\left\|\widetilde{\bar{\eta}}_{\Omega_{t}}\right\|_{0, \hat{\Omega}}$ consider the problem

$$
\begin{aligned}
& \operatorname{div} w=p_{\sigma \hat{\eta}} \widetilde{\bar{\eta}}_{\Omega_{t}},\left.\quad w_{3}\right|_{z_{3}=0}=\chi\left(z^{\prime}\right) \int_{\hat{\Omega}} p_{\sigma \hat{\eta}} \widetilde{\bar{\eta}}_{\Omega_{t}} d z, \\
& \left.w\right|_{\partial \hat{\Omega} \backslash \hat{S}}=0,\left.\quad w_{i}\right|_{z_{3}=0}=0, \quad i=1,2,
\end{aligned}
$$

where $\chi\left(z^{\prime}\right)$ is a smooth function such that $\int_{\hat{S}} \chi\left(z^{\prime}\right) d z^{\prime}=1, \chi\left(z^{\prime}\right) \geq 0$, $\left.\chi\right|_{\left|z^{\prime}\right|=d}=0,1 \leq 4 d^{2}|\chi|_{\infty, \hat{S}}$. Moreover, we assume that $\chi$ vanishes only in a neighbourhood of $\widehat{S}, \min _{\left|z^{\prime}\right| \leq d / 2} \chi\left(z^{\prime}\right)>0$ and $\chi\left(z^{\prime}\right) \leq c / d^{2}$. By [21] (Lemma 4.4) there exists a solution of (3.72) such that $w \in W_{2}^{1}(\widehat{\Omega})$ and

$$
\|w\|_{1, \hat{\Omega}} \leq C_{1}\left\|\widetilde{\bar{\eta}}_{\Omega_{t}}\right\|_{0, \hat{\Omega}} .
$$

Now, multiply (3.68) by $w$ and integrate over $\widehat{\Omega}$ to get

$$
\begin{aligned}
-\mu \int_{\hat{\Omega}} \Delta \widetilde{u} \cdot w d z+\int_{\hat{\Omega}} \nabla\left(p_{\sigma \hat{\eta}} \widetilde{\bar{\eta}}_{\Omega_{t}}\right) \cdot w d z & \\
& =\int_{\hat{\Omega}} \tilde{f} \cdot w d z+\nu \int_{\hat{\Omega}} \nabla \operatorname{div} \widetilde{u} \cdot w d z .
\end{aligned}
$$


Applying the same argument as in [21] (see the proof of Lemma 4.4) and using (3.73) we get

$$
\left\|\widetilde{\bar{\eta}}_{\Omega_{t}}\right\|_{0, \hat{\Omega}}^{2} \leq \varepsilon\left\|\widetilde{\bar{\eta}}_{\Omega_{t} z}\right\|_{0, \hat{\Omega}}^{2}+C_{1}\left(\|\widetilde{f}\|_{0, \hat{\Omega}}^{2}+\left\|\widetilde{u}_{z}\right\|_{0, \hat{\Omega}}^{2}+\|\operatorname{div} \widetilde{u}\|_{1, \hat{\Omega}}^{2}\right) .
$$

Now, instead of problem (3.68)-(3.70) we consider the problem

$$
\begin{aligned}
& -\mu \Delta \widetilde{u}_{i \tau}+\nabla_{z_{i}}\left(p_{\sigma \hat{\eta}} \widetilde{\bar{\eta}}_{\Omega_{t}}\right)_{, \tau}=\widetilde{f}_{i \tau}+\nu \nabla_{z_{i}} \operatorname{div} \widetilde{u}_{\tau}, \quad i=1,2,3, \\
& \partial_{z_{3}} \widetilde{u}_{i z}=\widetilde{h}_{i \tau}, \quad i=1,2 .
\end{aligned}
$$

Multiplying $(3.76)_{1}$ by $\widetilde{u}_{i \tau}$, summing over $i=1,2$ and integrating over $\widehat{\Omega}$ yields

$$
\begin{aligned}
\left\|\widetilde{u}_{z \tau}^{\prime}\right\|_{0, \hat{\Omega}}^{2} \leq & \varepsilon\left\|\widetilde{\bar{\eta}}_{\Omega_{t} \tau}\right\|_{0, \hat{\Omega}}^{2}+c\left(\left\|\widetilde{f}^{\prime}\right\|_{0, \hat{\Omega}}^{2}+\left\|\widetilde{h}_{z^{\prime}}^{\prime}\right\|_{0, \hat{\Omega}}^{2}\right)+C_{1}\left\|\operatorname{div} \widetilde{u}_{\tau}\right\|_{0, \hat{\Omega}}^{2} \\
& +C_{2}\left\|\widetilde{\bar{\eta}}_{\Omega_{t}}\right\|_{1, \hat{\Omega}}^{2}\left(\left\|\widetilde{\bar{\eta}}_{\Omega_{t}}\right\|_{2, \hat{\Omega}}^{2}+\left\|\widehat{\gamma}_{0}\right\|_{2, \hat{\Omega}}^{2}\right) .
\end{aligned}
$$

Next, consider the problem

$$
\operatorname{div} w_{1}=\left(p_{\sigma \hat{\eta}} \widetilde{\bar{\eta}}_{\Omega_{t}}\right)_{, \tau},\left.\quad w_{1}\right|_{\partial \hat{\Omega}}=0 .
$$

Since $\int_{\hat{\Omega}}\left(p_{\sigma \hat{\eta}} \widetilde{\bar{\eta}}_{\Omega_{t}}\right)_{, \tau} d z=0$ there exists a solution $w_{1} \in W_{2}^{1}(\widehat{\Omega})$ of problem (3.78) such that

$$
\left\|w_{1}\right\|_{1, \hat{\Omega}} \leq C_{1}\left[\left\|\widetilde{\bar{\eta}}_{\Omega_{t} \tau}\right\|_{0, \hat{\Omega}}+\left(\left\|\widehat{\eta}_{\sigma \tau}\right\|_{1, \hat{\Omega}}+\left\|\widehat{\gamma}_{0 \tau}\right\|_{1, \hat{\Omega}}\right)\left\|\widetilde{\bar{\eta}}_{\Omega_{t}}\right\|_{1, \hat{\Omega}}\right] .
$$

Multiplying (3.76) $)_{1}$ by $w_{1}$ and integrating over $\widehat{\Omega}$ gives

$$
\begin{aligned}
\left\|\widetilde{\bar{\eta}}_{\Omega_{t} \tau}\right\|_{0, \hat{\Omega}}^{2} \leq & C_{1}\left(\|\widetilde{f}\|_{0, \hat{\Omega}}^{2}+\left\|\widetilde{u}_{z \tau}\right\|_{0, \hat{\Omega}}^{2}+\|\operatorname{div} \widetilde{u}\|_{1, \hat{\Omega}}^{2}\right) \\
& +C_{2}\left(\left\|\widehat{\eta}_{\sigma}\right\|_{2, \hat{\Omega}}^{2}\left\|\widetilde{\bar{\eta}}_{\Omega_{t}}\right\|_{1, \hat{\Omega}}^{2}+\left\|\widehat{\gamma}_{0}\right\|_{2, \hat{\Omega}}^{2}\left\|\widetilde{\bar{\eta}}_{\Omega_{t}}\right\|_{1, \hat{\Omega}}^{2}\right) .
\end{aligned}
$$

Now we estimate $\left\|\widetilde{u}_{n n}^{\prime}\right\|_{0, \hat{\Omega}}^{2}$. From (3.68) we obtain

$$
\begin{aligned}
\left\|\widetilde{u}_{n n}^{\prime}\right\|_{0, \hat{\Omega}}^{2} \leq & C_{1}\left(\left\|\widetilde{\bar{\eta}}_{\Omega_{t} \tau}\right\|_{0, \hat{\Omega}}^{2}+\left\|\bar{\eta}_{\Omega_{t}}\right\|_{0, \hat{\Omega}}^{2}+\left\|\widetilde{\gamma}_{0 \tau}\right\|_{0, \hat{\Omega}}^{2}\right. \\
& \left.+\|\hat{\gamma}\|_{0, \hat{\Omega}}^{2}+\left\|\widetilde{u}_{z \tau}\right\|_{0, \hat{\Omega}}^{2}+\|\widetilde{f}\|_{0, \hat{\Omega}}^{2}+\|\operatorname{div} \widetilde{u}\|_{1, \hat{\Omega}}^{2}\right) .
\end{aligned}
$$

From the form of $\widetilde{f}$ and $\widetilde{h}^{\prime}$ we have

$$
\begin{aligned}
\|\widetilde{f}\|_{0, \hat{\Omega}}^{2} \leq & (\varepsilon+c d)\left(\left\|\widetilde{u}_{z z}\right\|_{0, \hat{\Omega}}^{2}+\left\|\widetilde{\bar{\eta}}_{\Omega_{t} z}\right\|_{0, \hat{\Omega}}^{2}\right) \\
& +C_{1}\left(|\widehat{u}|_{1,0, \hat{\Omega}}^{2}+\left\|\widehat{\bar{\eta}}_{\Omega_{t}}\right\|_{0, \hat{\Omega}}^{2}+\left\|\widehat{\gamma}_{0 z}\right\|_{0, \hat{\Omega}}^{2}+\|\widehat{\gamma}\|_{0, \hat{\Omega}}^{2}+\|\widetilde{g}\|_{0, \hat{\Omega}}^{2}\right) \\
& +C_{2}\left[\|\widehat{u}\|_{2, \hat{\Omega}}^{2}\left\|\int_{0}^{t} \widehat{u} d t^{\prime}\right\|_{3, \hat{\Omega}}^{2}+\left\|\widetilde{\bar{\eta}}_{\Omega_{t}}\right\|_{1, \hat{\Omega}}^{2}\left(\left\|\widehat{\bar{\eta}}_{\sigma}\right\|_{2, \hat{\Omega}}^{2}+\left\|\widehat{\gamma}_{0}\right\|_{2, \hat{\Omega}}^{2}\right)\right]
\end{aligned}
$$

and

$$
\left\|\widetilde{h}^{\prime}\right\|_{1, \hat{\Omega}}^{2} \leq C_{1}\left(\left\|\widetilde{u}_{3 z \tau}\right\|_{0, \hat{\Omega}}^{2}+\|\widetilde{u}\|_{2, \hat{\Omega}}^{2}\left\|\int_{0}^{t} \widehat{u} d t^{\prime}\right\|_{3, \hat{\Omega}}^{2}+\|\widehat{u}\|_{1, \hat{\Omega}}^{2}\right) .
$$


In order to estimate $\widetilde{\gamma}_{n n}$ we rewrite $(3.47)_{3}$ as

$$
\widehat{\eta} c_{v} \widetilde{\gamma}_{t}-\kappa \Delta \widetilde{\gamma}=\kappa \widehat{\nabla}^{2} \widetilde{\gamma}-\kappa \Delta \widetilde{\gamma}-\widehat{\Gamma} p_{\hat{\Gamma}} \widehat{\nabla} \cdot \widetilde{u}+\widehat{\eta} \widetilde{k}+k_{6} .
$$

Dividing (3.84) by $\widehat{\Gamma}$, multiplying the result by $\widetilde{\gamma}_{n n} J$ and integrating over $\widehat{\Omega}$ we get

$$
\begin{aligned}
& (3.85) \quad \frac{1}{2} \frac{d}{d t} \int_{\hat{\Omega}} \frac{\widehat{\eta} c_{v}}{\widehat{\Gamma}} \widetilde{\gamma}_{n}^{2} J d z+\frac{\kappa}{\theta^{*}}\left\|\widetilde{\gamma}_{n n}\right\|_{0, \hat{\Omega}}^{2} \\
& \leq(\varepsilon+c d)\left\|\widetilde{\gamma}_{n n}\right\|_{0, \hat{\Omega}}^{2}+\varepsilon\left(\left\|\widehat{\bar{\eta}}_{\Omega_{t} n}\right\|_{0, \hat{\Omega}}^{2}+\left\|\widetilde{\gamma}_{n t}\right\|_{0, \hat{\Omega}}^{2}\right) \\
& \quad+C_{1}\left(\|\widetilde{u}\|_{1, \hat{\Omega}}^{2}+\left\|\widehat{\gamma}_{0 t}\right\|_{0, \hat{\Omega}}^{2}+\left\|\widehat{\gamma}_{0 z}\right\|_{0, \hat{\Omega}}^{2}+\|\widehat{\gamma}\|_{0, \hat{\Omega}}^{2}+\left\|\widetilde{\gamma}_{z \tau}\right\|_{0, \hat{\Omega}}^{2}+\left\|\vartheta_{0 t}\right\|_{0, \Omega_{t}}^{2}+\|v\|_{1, \Omega_{t}}^{2}\right) \\
& \quad+C_{2}\left[\left\|\widehat{\gamma}_{0 t}\right\|_{1, \hat{\Omega}}^{2}\|\widehat{\gamma}\|_{2, \hat{\Omega}}^{2}+\|\widehat{\gamma}\|_{2, \hat{\Omega}}^{2}\left\|\int_{0}^{t} \widehat{u} d t^{\prime}\right\|_{3, \hat{\Omega}}^{2}+\|\widetilde{u}\|_{2, \hat{\Omega}}^{4}\right. \\
& \quad+\|\widehat{u}\|_{2, \hat{\Omega}}^{2}\left\|\int_{0}^{t} \widehat{u} d t^{\prime}\right\|_{3, \hat{\Omega}}^{2}+\|\widehat{\gamma}\|_{3, \hat{\Omega}}^{2}\|\widehat{u}\|_{2, \hat{\Omega}}^{2}+\left\|\widehat{\eta}_{\sigma t}\right\|_{1, \hat{\Omega}}^{2}\|\widehat{\gamma}\|_{2, \hat{\Omega}}^{2} \\
& \left.\quad+\|\widehat{\gamma}\|_{3, \hat{\Omega}}^{2}\left\|\int_{0}^{t} \widehat{u} d t^{\prime}\right\|_{2, \hat{\Omega}}^{2}+\|\widehat{\gamma}\|_{2, \hat{\Omega}}^{2}\left(\|v\|_{1, \Omega_{t}}^{2}+\left\|\vartheta_{0 t}\right\|_{0, \Omega_{t}}^{2}\right)\right] .
\end{aligned}
$$

Finally, we have

$$
\frac{d}{d t} \int_{\hat{\Omega}} \widehat{\eta} \widetilde{u}_{n}^{2} J d z \leq \varepsilon\left\|\widetilde{u}_{n t}\right\|_{0, \hat{\Omega}}^{2}+c\|u\|_{1, \hat{\Omega}}^{2} .
$$

Now, taking into account inequalities (3.60)-(3.62), (3.65), (3.67), (3.71), (3.72), (3.77), (3.80)-(3.83), (3.85) and (3.86) we get

$$
\begin{aligned}
\frac{1}{2} \frac{d}{d t} \int_{\hat{\Omega}}\left(\widehat{\eta} \widetilde{u}_{z}^{2}+\right. & \left.\frac{p_{\sigma \hat{\eta}}}{\widehat{\eta}} \widetilde{\bar{\eta}}_{\Omega_{t} z}^{2}+\frac{\widehat{\eta} c_{v}}{\widehat{\Gamma}} \widetilde{\gamma}_{z}^{2}\right) J d z \\
& +c_{0}\left(\left\|\widetilde{u}_{z}\right\|_{1, \hat{\Omega}}^{2}+\left\|\widetilde{\bar{\eta}}_{\Omega_{t}}\right\|_{0, \hat{\Omega}}^{2}+\left\|\widetilde{\eta}_{\sigma z}\right\|_{0, \hat{\Omega}}^{2}+\left\|\widetilde{\gamma}_{z z}\right\|_{0, \hat{\Omega}}^{2}\right) \\
\leq & \varepsilon\left(\left\|\widetilde{u}_{z t}\right\|_{0, \hat{\Omega}}^{2}+\left\|\widetilde{\gamma}_{0 z t}\right\|_{0, \hat{\Omega}}^{2}\right) \\
& +C_{1}\left(|\widehat{u}|_{1,0, \hat{\Omega}}^{2}+\left\|\widehat{\bar{\eta}}_{\Omega_{t}}\right\|_{0, \hat{\Omega}}^{2}+\|\widehat{\gamma}\|_{0, \hat{\Omega}}^{2}+\left\|\widehat{\gamma}_{0 z}\right\|_{0, \hat{\Omega}}^{2}\right. \\
& \left.+\left\|\widehat{\gamma}_{0 t}\right\|_{0, \hat{\Omega}}^{2}+\|\widetilde{g}\|_{0, \hat{\Omega}}^{2}+\|\widetilde{k}\|_{0, \hat{\Omega}}^{2}+\left\|\widetilde{\Gamma}_{1}\right\|_{2, \hat{\Omega}}^{2}\right) \\
& +C_{2}\left[\left(X_{2}(\widehat{\Omega})+\int_{0}^{t}\|\widehat{u}\|_{3, \hat{\Omega}}^{2} d t^{\prime}\right) \cdot Y_{2}(\widehat{\Omega})\right. \\
& \left.+\|\widehat{\gamma}\|_{2, \hat{\Omega}}^{2}\left(\left\|\vartheta_{0 t}\right\|_{0, \Omega_{t}}^{2}+\|v\|_{1, \Omega_{t}}^{2}+\|\widehat{\gamma}\|_{2, \hat{\Omega}}^{2}\right)\right]
\end{aligned}
$$


Going back to the variables $\xi$ in (3.87), summing over all neighbourhoods of the partition of unity (where we use (3.59) for interior subdomains), assuming that $\varepsilon$ and $d$ are sufficiently small and using (3.34), we obtain (3.48).

LEMMA 3.5. Let $v, \varrho, \vartheta_{0}$ be a sufficiently smooth solution of problem (3.3). Then

$$
\begin{aligned}
& \frac{1}{2} \frac{d}{d t} \int_{\Omega_{t}}\left(\varrho v_{x x}^{2}+\frac{p_{\sigma \varrho}}{\varrho} \varrho_{\sigma x x}^{2}+\frac{\varrho c_{v}}{\theta} \vartheta_{0 x x}^{2}\right) d x \\
& \quad+c_{0}\left(\left\|v_{x x}\right\|_{1, \Omega_{t}}^{2}+\left\|\varrho_{\sigma x}\right\|_{1, \Omega_{t}}^{2}+\left\|\vartheta_{0 x x x}\right\|_{0, \Omega_{t}}^{2}\right) \\
& \leq \varepsilon\left(\left\|v_{x x t}\right\|_{0, \Omega_{t}}^{2}+\left\|\vartheta_{0 x x t}\right\|_{0, \Omega_{t}}^{2}\right) \\
& \quad+C_{1}\left(|v|_{2,1, \Omega_{t}}^{2}+\left\|\varrho_{\sigma x}\right\|_{0, \Omega_{t}}^{2}+\left\|\varrho_{\sigma t}\right\|_{0, \Omega_{t}}^{2}+\left\|\bar{\varrho}_{\Omega_{t}}\right\|_{0, \Omega_{t}}^{2}+\left\|\vartheta_{0 x}\right\|_{1, \Omega_{t}}^{2}\right. \\
& \left.\quad+\left\|\vartheta_{0 t}\right\|_{1, \Omega_{t}}^{2}+\|\vartheta\|_{0, \Omega_{t}}^{2}+\|f\|_{1, \Omega_{t}}^{2}+\|r\|_{1, \Omega_{t}}^{2}+\left\|\theta_{1}\right\|_{3, \Omega_{t}}^{2}\right) \\
& \quad+C_{2}\left(X_{3}+\int_{0}^{t}\|v\|_{4, \Omega_{t^{\prime}}}^{2} d t^{\prime}\right)\left(1+X_{3}\right) Y_{3},
\end{aligned}
$$

where

$$
\begin{gathered}
v_{x x}^{2}=\sum_{i, j, k=1}^{3} v_{i x_{j} x_{k}}^{2}, \quad \varrho_{\sigma x x}^{2}=\sum_{j, k=1}^{3} \varrho_{\sigma x_{j} x_{k}}^{2}, \quad \vartheta_{0 x x}^{2}=\sum_{j, k=1}^{3} \vartheta_{0 x_{j} x_{k}}^{2}, \\
X_{3}=\|v\|_{3, \Omega_{t}}^{2}+\left\|v_{t}\right\|_{1, \Omega_{t}}^{2}+\left|\varrho_{\sigma}\right|_{2,1, \Omega_{t}}^{2}+\left|\vartheta_{0}\right|_{2,1, \Omega_{t}}^{2}+\left\|\vartheta_{0}\right\|_{3, \Omega_{t}}^{2}+\left\|\bar{\varrho}_{\Omega_{t}}\right\|_{0, \Omega_{t}}^{2} \\
Y_{3}=\|v\|_{4, \Omega_{t}}^{2}+\left\|v_{t}\right\|_{1, \Omega_{t}}^{2}+\left\|\varrho_{\sigma x}\right\|_{2, \Omega_{t}}^{2}+\left|\varrho_{\sigma}\right|_{2,1, \Omega_{t}}^{2}+\left\|\vartheta_{0 x}\right\|_{3, \Omega_{t}}^{2}+\left\|\vartheta_{0 t}\right\|_{1, \Omega_{t}}^{2} \\
+\left\|\bar{\varrho}_{\Omega_{t}}\right\|_{0, \Omega_{t}}^{2}+\|\vartheta\|_{0, \Omega_{t}}^{2} .
\end{gathered}
$$

Pr o of. The proof is similar to that of Lemma 3.4. We use the introduced partition of unity. Differentiating $(3.46)_{1}$ and $(3.46)_{3}$ (divided by $\Gamma$ ) twice with respect to $\xi$, multiplying the results by $u_{\xi \xi} A$ and $\widetilde{\gamma}_{\xi \xi} A$, respectively, next integrating over $\widetilde{\Omega}$ and summing up we get

$$
\begin{aligned}
\frac{1}{2} \frac{d}{d t} \int_{\tilde{\Omega}}\left(\eta \widetilde{u}_{\xi \xi}^{2}\right. & \left.+\frac{p_{\sigma \eta}}{\eta} \widetilde{\bar{\eta}}_{\Omega_{t} \xi \xi}+\frac{\eta c_{v}}{\Gamma} \widetilde{\gamma}_{\xi \xi}^{2}\right) A d \xi \\
& +\frac{1}{2} \mu\left\|\widetilde{u}_{\xi \xi}\right\|_{1, \tilde{\Omega}}^{2}+\left\|\widetilde{\bar{\eta}}_{\Omega_{t}}\right\|_{2, \tilde{\Omega}}^{2}+\frac{\kappa}{\theta^{*}}\left\|\widetilde{\gamma}_{\xi \xi \xi}\right\|_{0, \tilde{\Omega}}^{2} \\
\leq & \varepsilon\left(\left\|\widetilde{u}_{\xi \xi \xi}\right\|_{0, \tilde{\Omega}}^{2}+\left\|\widetilde{\gamma}_{\xi \xi \xi}\right\|_{0, \tilde{\Omega}}^{2}+\left\|\widetilde{\eta}_{\sigma \xi \xi}\right\|_{0, \tilde{\Omega}}^{2}\right) \\
& +C_{1}\left(|u|_{2,1, \tilde{\Omega}}^{2}+\left\|\gamma_{0 \xi}\right\|_{1, \tilde{\Omega}}^{2}+\|\gamma\|_{0, \tilde{\Omega}}^{2}+\left\|\eta_{\sigma \xi}\right\|_{0, \tilde{\Omega}}^{2}\right.
\end{aligned}
$$




$$
\begin{aligned}
& \left.+\left\|\bar{\eta}_{\Omega_{t}}\right\|_{0, \tilde{\Omega}}^{2}+\left\|\vartheta_{0 t}\right\|_{0, \Omega_{t}}^{2}+\|v\|_{1, \Omega_{t}}^{2}+\|\widetilde{g}\|_{1, \tilde{\Omega}}^{2}+\|\widetilde{k}\|_{1, \tilde{\Omega}}^{2}\right) \\
& +C_{2}\left[\left(X_{3}(\widetilde{\Omega})+\int_{0}^{t}\|u\|_{3, \tilde{\Omega}}^{2} d t^{\prime}\right)\left(1+X_{3}(\widetilde{\Omega})\right) Y_{3}(\widetilde{\Omega})\right. \\
& \left.+\|\gamma\|_{3, \tilde{\Omega}}^{2}\left(\left\|\vartheta_{0 t}\right\|_{0, \Omega_{t}}^{2}+\|v\|_{1, \Omega_{t}}^{2}\right)\right]
\end{aligned}
$$

where we have used equation (3.52), Lemma 5.1 of [21] and the estimate for the solution $u, \bar{\eta}_{\Omega_{t}}$ of the Stokes problem (3.57), i.e. the estimate of $\|\widetilde{u}\|_{3, \tilde{\Omega}}$ and $\left\|\widetilde{\bar{\eta}}_{\Omega_{t}}\right\|_{2, \tilde{\Omega}}$, respectively and

$$
\begin{aligned}
X_{3}(\widetilde{\Omega})= & \|u\|_{3, \tilde{\Omega}}^{2}+\left\|u_{t}\right\|_{1, \tilde{\Omega}}^{2} \\
& +\left|\eta_{\sigma}\right|_{2,1, \tilde{\Omega}}^{2}+\left\|\gamma_{0}\right\|_{3, \tilde{\Omega}}^{2}+\left|\gamma_{0}\right|_{2,1, \tilde{\Omega}}^{2}+\left\|\bar{\eta}_{\Omega_{t}}\right\|_{0, \tilde{\Omega}}^{2}, \\
Y_{3}(\widetilde{\Omega})= & \|u\|_{4, \tilde{\Omega}}^{2}+\left\|u_{t}\right\|_{1, \tilde{\Omega}}^{2}+\left\|\eta_{\sigma x}\right\|_{2, \tilde{\Omega}}^{2}+\left|\eta_{\sigma}\right|_{2,1, \tilde{\Omega}}^{2} \\
& +\left\|\gamma_{0 x}\right\|_{3, \tilde{\Omega}}^{2}+\left\|\gamma_{0 t}\right\|_{1, \tilde{\Omega}}^{2}+\left\|\bar{\eta}_{\Omega_{t}}\right\|_{0, \Omega_{t}}^{2}+\|\gamma\|_{0, \Omega_{t}}^{2} .
\end{aligned}
$$

In the same way as (3.60) we obtain the following inequality:

$$
\begin{aligned}
& \frac{1}{2} \frac{d}{d t} \int_{\hat{\Omega}}\left(\widehat{\eta} \widetilde{u}_{\tau \tau}^{2}+\frac{p_{\sigma \hat{\eta}}}{\widehat{\eta}} \widetilde{\eta}_{\Omega_{t} \tau \tau}^{2}+\frac{\widehat{\eta} c_{v}}{\widehat{\Gamma}} \widetilde{\gamma}_{\tau \tau}^{2}\right) J d z+\frac{1}{2} \mu\left\|\widetilde{u}_{\tau \tau}\right\|_{1, \hat{\Omega}}^{2}+\frac{\kappa}{\theta^{*}}\left\|\widetilde{\gamma}_{\tau \tau z}\right\|_{0, \hat{\Omega}}^{2} \\
& \leq \varepsilon\left(\left\|\widehat{u}_{z z z}\right\|_{0, \hat{\Omega}}^{2}+\left\|\widehat{\gamma}_{0 z z z}\right\|_{0, \hat{\Omega}}^{2}+\left\|\widehat{\eta}_{\sigma z z}\right\|_{0, \hat{\Omega}}^{2}\right) \\
& +C_{1}\left(|\widehat{u}|_{2,1, \hat{\Omega}}^{2}+\left\|\widehat{\gamma}_{0 z}\right\|_{1, \hat{\Omega}}^{2}+\|\widehat{\gamma}\|_{0, \hat{\Omega}}^{2}+\left\|\eta_{\sigma z}\right\|_{0, \hat{\Omega}}^{2}+\left\|\bar{\eta}_{\Omega_{t}}\right\|_{0, \hat{\Omega}}^{2}\right. \\
& \left.+\left\|\vartheta_{0 t}\right\|_{0, \Omega_{t}}^{2}+\|v\|_{1, \Omega_{t}}^{2}+\|\widetilde{g}\|_{1, \hat{\Omega}}^{2}+\|\widetilde{k}\|_{1, \hat{\Omega}}^{2}+\left\|\widetilde{\Gamma}_{1}\right\|_{3, \hat{\Omega}}^{2}\right) \\
& +C_{2}\left[\left(X_{3}(\widehat{\Omega})+\int_{0}^{t}\|\widehat{u}\|_{3, \hat{\Omega}}^{2} d t^{\prime}\right)\left(1+X_{3}(\widehat{\Omega})\right) Y_{3}(\widehat{\Omega})\right. \\
& \left.+\|\widehat{\gamma}\|_{3, \hat{\Omega}}^{2}\left(\left\|\vartheta_{0 t}\right\|_{0, \Omega_{t}}^{2}+\|v\|_{1, \Omega_{t}}^{2}+\|\widehat{\gamma}\|_{3, \hat{\Omega}}^{2}\right)\right],
\end{aligned}
$$

where we have used the boundary conditions $(3.47)_{4}$ and $(3.47)_{5}$, and where $X_{3}(\widehat{\Omega})$ and $Y_{3}(\widehat{\Omega})$ are defined by $(3.90)$ with $\widetilde{\Omega}, u, \bar{\eta}_{\Omega_{t}}, \gamma$ replaced by $\widehat{\Omega}, \widehat{u}$, $\widehat{\bar{\eta}}_{\Omega_{t}}, \widehat{\gamma}$, respectively.

Differentiating the third component of (3.64) with respect to $\tau$, multiplying the result by $\widetilde{\bar{\eta}}_{\Omega_{t} n \tau} J$ and integrating over $\widehat{\Omega}$ yields

$$
\begin{aligned}
& \frac{1}{2} \frac{d}{d t} \int_{\hat{\Omega}} \frac{p_{\sigma \hat{\eta}}}{\widehat{\eta}} \widetilde{\bar{\eta}}_{\Omega_{t} n \tau}^{2} J d z+c_{0}\left\|\widetilde{\bar{\eta}}_{\Omega_{t} n \tau}\right\|_{0, \hat{\Omega}}^{2} \\
& \leq(\varepsilon+c d)\left(\left\|\widetilde{\bar{\eta}}_{\Omega_{t} z z}\right\|_{0, \hat{\Omega}}^{2}+\left\|\widehat{u}_{z z z}\right\|_{0, \hat{\Omega}}^{2}\right)+C_{1}\left(|\widehat{u}|_{2,1, \hat{\Omega}}^{2}+\left\|\widehat{\bar{\eta}}_{\Omega_{t}}\right\|_{0, \hat{\Omega}}^{2}+\left\|\widehat{\eta}_{\sigma z}\right\|_{0, \hat{\Omega}}^{2}\right.
\end{aligned}
$$




$$
\begin{aligned}
& \left.+\|\widehat{\gamma}\|_{0, \hat{\Omega}}^{2}+\left\|\widehat{\gamma}_{0 z}\right\|_{1, \hat{\Omega}}^{2}+\left\|\widetilde{u}_{z \tau}\right\|_{0, \hat{\Omega}}^{2}+\left\|\vartheta_{0 t}\right\|_{0, \Omega_{t}}^{2}+\|v\|_{1, \Omega_{t}}^{2}+\|\widetilde{g}\|_{1, \hat{\Omega}}^{2}\right) \\
& +C_{2}\left(X_{3}(\widehat{\Omega})+\int_{0}^{t}\|\widehat{u}\|_{3, \hat{\Omega}^{2}}^{2} d t^{\prime}\right)\left(1+X_{3}(\widehat{\Omega})\right) Y_{3}(\widehat{\Omega}) .
\end{aligned}
$$

Next, differentiating the third component of (3.66) with respect to $\tau$, multiplying the result by $\widetilde{u}_{3 n n \tau} J$ and integrating over $\widehat{\Omega}$ gives

$$
\begin{aligned}
\frac{1}{2} \frac{d}{d t} \int_{\hat{\Omega}} & \hat{\eta}\left|\widetilde{u}_{3 n \tau}\right|^{2} J d z+c_{0}\left\|\widetilde{u}_{3 n n \tau}\right\|_{0, \hat{\Omega}}^{2} \\
\leq & (\varepsilon+c d)\left(\left\|\widetilde{u}_{z z z}\right\|_{0, \hat{\Omega}}^{2}+\left\|\widetilde{\bar{\eta}}_{\Omega_{t} z z}\right\|_{0, \hat{\Omega}}^{2}\right) \\
& +C_{1}\left(\left\|\widetilde{u}_{z \tau \tau}\right\|_{0, \hat{\Omega}}^{2}+\left\|\widetilde{\bar{\eta}}_{\Omega_{t} n \tau}\right\|_{0, \hat{\Omega}}^{2}+\|\widehat{u}\|_{2, \hat{\Omega}}^{2}+\left\|\widetilde{u}_{t}\right\|_{1, \hat{\Omega}}^{2}\right. \\
& \left.+\left\|\widehat{\bar{\eta}}_{\Omega_{t}}\right\|_{0, \hat{\Omega}}^{2}+\left\|\widehat{\eta}_{\sigma z}\right\|_{0, \hat{\Omega}}^{2}+\|\widehat{\gamma}\|_{0, \hat{\Omega}}^{2}+\left\|\widehat{\gamma}_{0 z}\right\|_{1, \hat{\Omega}}^{2}+\|\widetilde{g}\|_{1, \hat{\Omega}}^{2}\right) \\
& +C_{2}\left(X_{3}(\widehat{\Omega})+\int_{0}^{t}\|\widehat{u}\|_{3, \hat{\Omega}}^{2} d t^{\prime}\right)\left(1+X_{3}(\widehat{\Omega})\right) Y_{3}(\widehat{\Omega}) .
\end{aligned}
$$

Similarly, we obtain the estimate

$$
\begin{aligned}
& \frac{1}{2} \frac{d}{d t} \int_{\hat{\Omega}} \frac{\widehat{\eta} c_{v}}{\widehat{\Gamma}} \widetilde{\gamma}_{n \tau}^{2} J d z+\frac{\kappa}{\theta^{*}}\left\|\widetilde{\gamma}_{n n \tau}\right\|_{0, \hat{\Omega}}^{2} \\
& \leq(\varepsilon+c d)\left(\left\|\widetilde{\gamma}_{z z z}\right\|_{0, \hat{\Omega}}^{2}+\left\|\widehat{\eta}_{\sigma z z}\right\|_{0, \hat{\Omega}}^{2}\right)+C_{1}\left(\|\widetilde{u}\|_{2, \hat{\Omega}}^{2}+\left\|\widehat{\gamma}_{0 z}\right\|_{1, \hat{\Omega}}^{2}+\|\widehat{\gamma}\|_{0, \hat{\Omega}}^{2}\right. \\
& \left.\quad+\left\|\widehat{\gamma}_{0 t}\right\|_{0, \hat{\Omega}}^{2}+\left\|\vartheta_{0 t}\right\|_{0, \Omega_{t}}^{2}+\|v\|_{1, \Omega_{t}}^{2}+\|\widetilde{k}\|_{0, \hat{\Omega}}\right) \\
& \quad+C_{2}\left[\left(X_{3}(\widehat{\Omega})+\int_{0}^{t}\|\widehat{u}\|_{3, \hat{\Omega}}^{2} d t^{\prime}\right)\left(1+X_{3}(\widehat{\Omega})\right) Y_{3}(\widehat{\Omega})\right. \\
& \left.\quad+\left(\left\|\widehat{\eta}_{\sigma}\right\|_{1, \hat{\Omega}}^{2}+\left\|\widehat{\gamma}_{0}\right\|_{1, \hat{\Omega}}^{2}\right)\left(\left\|\vartheta_{0 t}\right\|_{0, \Omega_{t}}^{2}+\|v\|_{1, \Omega_{t}}^{2}\right)\right] .
\end{aligned}
$$

Next, using problem (3.68)-(3.69) we have

$$
\begin{aligned}
&\left\|\widetilde{u}_{z \tau \tau}^{\prime}\right\|_{0, \hat{\Omega}}^{2}+\left\|\widetilde{\bar{\eta}}_{\Omega_{t} \tau \tau}\right\|_{0, \hat{\Omega}}^{2} \\
& \leq(\varepsilon+c d)\left(\left\|\widetilde{u}_{z z z}\right\|_{0, \hat{\Omega}}^{2}+\left\|\widehat{\eta}_{\sigma z z}\right\|_{0, \hat{\Omega}}^{2}\right) \\
&+C_{1}\left(\left\|\operatorname{div} \widetilde{u}_{\tau}\right\|_{1, \hat{\Omega}}^{2}+\left\|\widetilde{u}_{t}\right\|_{0, \hat{\Omega}}^{2}+\|\widehat{u}\|_{2, \hat{\Omega}}^{2}+\left\|\widehat{\gamma}_{0 z}\right\|_{1, \hat{\Omega}}^{2}\right. \\
&\left.+\|\widehat{\gamma}\|_{0, \hat{\Omega}}^{2}+\left\|\widehat{\eta}_{\sigma z}\right\|_{0, \hat{\Omega}}^{2}+\left\|\widehat{\bar{\eta}}_{\Omega_{t}}\right\|_{0, \hat{\Omega}}^{2}+\|\widetilde{g}\|_{1, \hat{\Omega}}^{2}\right) \\
&+C_{2}\left(X_{3}(\widehat{\Omega})+\int_{0}^{t}\|\widehat{u}\|_{3, \hat{\Omega}}^{2} d t^{\prime}\right)\left(1+X_{3}(\widehat{\Omega})\right) Y_{3}(\widehat{\Omega})
\end{aligned}
$$


and

$$
\begin{aligned}
\left\|\widetilde{u}_{n n \tau}^{\prime}\right\|_{0, \hat{\Omega}}^{2} \leq & (\varepsilon+c d)\left(\left\|\widehat{u}_{z z z}\right\|_{0, \hat{\Omega}}^{2}+\left\|\widehat{\bar{\eta}}_{\Omega_{t} z z}\right\|_{0, \hat{\Omega}}^{2}\right) \\
& +C_{1}\left(\|\widehat{u}\|_{2, \hat{\Omega}}^{2}+\left\|\widetilde{u}_{t}\right\|_{1, \hat{\Omega}}^{2}+\left\|\widehat{\eta}_{\sigma z}\right\|_{0, \hat{\Omega}}^{2}\right. \\
& \left.+\left\|\widehat{\bar{\eta}}_{\Omega_{t}}\right\|_{0, \hat{\Omega}}^{2}+\left\|\widehat{\gamma}_{0 z}\right\|_{1, \hat{\Omega}}^{2}+\|\widehat{\gamma}\|_{0, \hat{\Omega}}^{2}+\|\widetilde{g}\|_{1, \hat{\Omega}}^{2}\right) \\
& +C_{2}\left(X_{3}(\widehat{\Omega})+\int_{0}^{t}\|\widehat{u}\|_{3, \hat{\Omega}}^{2} d t^{\prime}\right)\left(1+X_{3}(\widehat{\Omega})\right) Y_{3}(\widehat{\Omega}) .
\end{aligned}
$$

Hence, taking into account (3.91)-(3.96) we get

$$
\begin{aligned}
& \frac{1}{2} \frac{d}{d t} \int_{\hat{\Omega}}\left[\widehat{\eta}\left(\widetilde{u}_{\tau \tau}^{2}+\widetilde{u}_{3 n \tau}^{2}\right)+\frac{p_{\sigma \hat{\eta}}}{\widehat{\eta}}\left(\widetilde{\bar{\eta}}_{\Omega_{t} \tau \tau}^{2}+\widetilde{\bar{\eta}}_{\Omega_{t} n \tau}^{2}\right)+\frac{\widehat{\eta} c_{v}}{\widehat{\Gamma}}\left(\widetilde{\gamma}_{\tau \tau}^{2}+\widetilde{\gamma}_{n \tau}^{2}\right)\right] J d z \\
& \quad+c_{0}\left(\left\|\widetilde{u}_{\tau}\right\|_{2, \hat{\Omega}}^{2}+\left\|\widetilde{\bar{\eta}}_{\Omega_{t} \tau}\right\|_{1, \hat{\Omega}}^{2}+\left\|\widetilde{\gamma}_{z z \tau}\right\|_{0, \hat{\Omega}}^{2}\right) \\
& \quad(\varepsilon+c d)\left(\left\|\widehat{u}_{z z z}\right\|_{0, \hat{\Omega}}^{2}+\left\|\widehat{\gamma}_{0 z z z}\right\|_{0, \hat{\Omega}}^{2}+\left\|\widehat{\eta}_{\sigma z z}\right\|_{0, \hat{\Omega}}^{2}\right) \\
& \quad+C_{1}\left(\mid \widehat{u}_{2,1, \hat{\Omega}}^{2}+\left\|\widehat{\gamma}_{0, \hat{\Omega}}^{2}+\right\| \widehat{\gamma}_{0 z}\left\|_{1, \hat{\Omega}}^{2}+\right\| \widehat{\gamma}_{0 t}\left\|_{1, \hat{\Omega}}^{2}+\right\| \widehat{\bar{\eta}}_{\Omega_{t}} \|_{0, \hat{\Omega}}^{2}\right. \\
& \left.\quad+\left\|\widehat{\eta}_{\sigma z}\right\|_{0, \hat{\Omega}}^{2}+\left\|\vartheta_{0 t}\right\|_{0, \Omega_{t}}^{2}+\|v\|_{1, \Omega_{t}}^{2}+\|\tilde{g}\|_{1, \hat{\Omega}}^{2}+\|\widetilde{k}\|_{1, \tilde{\Omega}}^{2}\right) \\
& \quad+C_{2}\left[\left(X_{3}(\widehat{\Omega})+\int_{0}^{t}\|\widehat{u}\|_{3, \hat{\Omega}}^{2} d t^{\prime}\right)\left(1+X_{3}(\widehat{\Omega})\right) Y_{3}(\widehat{\Omega})\right. \\
& \left.\quad+\left(\left\|\widehat{\eta}_{\sigma}\right\|_{1, \hat{\Omega}}^{2}+\left\|\widehat{\gamma}_{0}\right\|_{1, \hat{\Omega}}^{2}+\| \widehat{\gamma}_{3, \hat{\Omega}}^{2}\right)\left(\left\|\vartheta_{0 t}\right\|_{0, \Omega_{t}}^{2}+\|v\|_{1, \Omega_{t}}^{2}\right)+\|\widehat{\gamma}\|_{3, \hat{\Omega}}^{4}\right] .
\end{aligned}
$$

Differentiating the third component of (3.64) with respect to $n$, multiplying the result by $\widetilde{\bar{\eta}}_{\Omega_{t} n n} J$ and next integrating over $\widehat{\Omega}$ implies

$$
\begin{aligned}
& \frac{1}{2} \frac{d}{d t} \int_{\hat{\Omega}} \frac{(\mu+\nu) p_{\sigma \hat{\eta}}}{\widehat{\eta}} \overline{\bar{\eta}}_{\Omega_{t} n n}^{2} J d z+c_{0}\left\|\widetilde{\bar{\eta}}_{\Omega_{t} n n}\right\|_{0, \hat{\Omega}}^{2} \\
& \leq(\varepsilon+c d)\left(\left\|\widetilde{u}_{z z z}\right\|_{0, \hat{\Omega}}^{2}+\left\|\widetilde{\bar{\eta}}_{\Omega_{t} z z}\right\|_{0, \hat{\Omega}}^{2}\right) \\
&+C_{1}\left(\mid \widehat{u}_{2,1, \hat{\Omega}}^{2}+\left\|\widetilde{u}_{\tau}\right\|_{2, \hat{\Omega}}^{2}+\left\|\widehat{\bar{\eta}}_{\Omega_{t}}\right\|_{0, \hat{\Omega}}^{2}+\left\|\widehat{\eta}_{\sigma z}\right\|_{0, \hat{\Omega}}^{2}\right. \\
&\left.+\|\widehat{\gamma}\|_{0, \hat{\Omega}}^{2}+\left\|\widehat{\gamma}_{0 z}\right\|_{1, \hat{\Omega}}^{2}+\left\|\vartheta_{0 t}\right\|_{0, \Omega_{t}}^{2}+\|v\|_{1, \Omega_{t}}^{2}+\|\widetilde{g}\|_{1, \hat{\Omega}}^{2}\right) \\
&+C_{2}\left(X_{3}(\widehat{\Omega})+\int_{0}^{t}\|\widehat{u}\|_{3, \hat{\Omega}}^{2} d t^{\prime}\right)\left(1+X_{3}(\widehat{\Omega})\right) Y_{3}(\widehat{\Omega}) .
\end{aligned}
$$

Now, we rewrite $(3.47)_{1}$ in the form 
(3.99) $(\mu+\nu) \nabla_{z_{i}} \operatorname{div} \widetilde{u}=-\mu\left(\Delta \widetilde{u}_{i}-\nabla_{z_{i}} \operatorname{div} \widetilde{u}\right)+\widehat{\eta} \widetilde{u}_{i t}-\widehat{\eta} \widetilde{g}_{i}-k_{4}^{i}$

$$
\begin{aligned}
& -\left(\mu \nabla^{2} \widetilde{u}_{i}+\nu \nabla_{z_{i}} \operatorname{div} \widetilde{u}-\mu \widehat{\nabla}^{2} \widetilde{u}_{i}-\nu \widehat{\nabla} \widehat{\operatorname{div}} \widetilde{u}\right) \\
& -\left(p_{\sigma \hat{\eta}} \widehat{\nabla}_{i} \widetilde{\bar{\eta}}_{\Omega_{t}}-p_{\sigma \hat{\eta}} \widehat{\bar{\eta}}_{\Omega_{t}} \widehat{\nabla}_{i} \widehat{\zeta}+\widetilde{p}_{\sigma \hat{\Gamma}} \widehat{\nabla}_{i} \widehat{\gamma}_{0}\right) .
\end{aligned}
$$

Differentiating the third component of (3.99) with respect to $n$ gives

(3.100) $\quad\left\|(\operatorname{div} \widetilde{u})_{, n n}\right\|_{0, \hat{\Omega}}^{2}$

$$
\begin{aligned}
\leq & (\varepsilon+c d)\|\widetilde{u}\|_{3, \hat{\Omega}}^{2}+C_{1}\left(|\widehat{u}|_{2,1, \hat{\Omega}}^{2}+\left\|\widetilde{u}_{\tau}\right\|_{2, \hat{\Omega}}^{2}+\|\widehat{\gamma}\|_{0, \hat{\Omega}}^{2}\right. \\
& \left.+\left\|\widehat{\gamma}_{0 z}\right\|_{0, \hat{\Omega}}^{2}+\left\|\widehat{\gamma}_{0 n n}\right\|_{0, \hat{\Omega}}^{2}+\left\|\widehat{\bar{\eta}}_{\Omega_{t}}\right\|_{0, \hat{\Omega}}^{2}+\left\|\widehat{\eta}_{\sigma z}\right\|_{0, \hat{\Omega}}^{2}+\left\|\widehat{\bar{\eta}}_{\Omega_{t} n n}\right\|_{0, \hat{\Omega}}^{2}+\|\widetilde{g}\|_{0, \hat{\Omega}}^{2}\right) \\
& +C_{2}\left(X_{3}(\widehat{\Omega})+\int_{0}^{t}\|\widehat{u}\|_{3, \hat{\Omega}}^{2} d t^{\prime}\right)\left(1+X_{3}(\widehat{\Omega})\right) Y_{3}(\widehat{\Omega}) .
\end{aligned}
$$

Next, differentiating (3.68) with respect to $n$ yields

(3.101) $\left\|\widetilde{u}_{n n n}\right\|_{0, \hat{\Omega}}^{2}$

$$
\begin{aligned}
\leq & (\varepsilon+c d)\|\widetilde{u}\|_{3, \hat{\Omega}}^{2}+C_{1}\left(|\widehat{u}|_{2,1, \hat{\Omega}}^{2}+\left\|\widetilde{u}_{\tau \tau}\right\|_{1, \hat{\Omega}}^{2}+\left\|(\operatorname{div} \widetilde{u})_{, n}\right\|_{1, \hat{\Omega}}^{2}\right. \\
& \left.+\left\|\widetilde{\bar{\eta}}_{\Omega_{t} n}\right\|_{1, \hat{\Omega}}^{2}+\left\|\widehat{\bar{\eta}}_{\Omega_{t}}\right\|_{0, \hat{\Omega}}^{2}+\left\|\widehat{\eta}_{\sigma z}\right\|_{0, \hat{\Omega}}^{2}+\|\widehat{\gamma}\|_{0, \hat{\Omega}}^{2}+\left\|\widehat{\gamma}_{0 z}\right\|_{1, \hat{\Omega}}^{2}+\|\widetilde{g}\|_{1, \hat{\Omega}}^{2}\right) \\
& +C_{2}\left(X_{3}(\widehat{\Omega})+\int_{0}^{t}\|\widehat{u}\|_{3, \hat{\Omega}}^{2} d t^{\prime}\right)\left(1+X_{3}(\widehat{\Omega})\right) Y_{3}(\widehat{\Omega}) .
\end{aligned}
$$

In order to estimate $\left\|\widetilde{\gamma}_{n n n}\right\|_{0, \hat{\Omega}}^{2}$ we use (3.84). We get

(3.102) $\left\|\widetilde{\gamma}_{n n n}\right\|_{0, \hat{\Omega}}^{2} \leq(\varepsilon+c d)\|\widetilde{\gamma}\|_{3, \hat{\Omega}}^{2}+C_{1}\left(\|\widehat{u}\|_{2, \hat{\Omega}}^{2}+\left\|\widehat{\bar{\eta}}_{\Omega_{t}}\right\|_{0, \hat{\Omega}}^{2}+\left\|\widehat{\eta}_{\sigma z}\right\|_{0, \hat{\Omega}}^{2}\right.$

$$
\begin{aligned}
& \left.+\|\widehat{\gamma}\|_{0, \hat{\Omega}}^{2}+\left\|\widehat{\gamma}_{0 z}\right\|_{1, \hat{\Omega}}^{2}+\left\|\widehat{\gamma}_{0 t}\right\|_{1, \hat{\Omega}}^{2}+\left\|\vartheta_{0 t}\right\|_{0, \Omega_{t}}^{2}+\|v\|_{1, \Omega_{t}}^{2}\right) \\
& +C_{2}\left(X_{3}(\widehat{\Omega})+\int_{0}^{t}\|\widehat{u}\|_{3, \hat{\Omega}}^{2} d t^{\prime}\right)\left(1+X_{3}(\widehat{\Omega})\right) Y_{3}(\widehat{\Omega}) .
\end{aligned}
$$

Finally, we have

$$
\frac{1}{2} \frac{d}{d t} \int_{\hat{\Omega}} \widehat{\eta} \widetilde{u}_{z z}^{2} J d z \leq \varepsilon\left\|\widetilde{u}_{z z t}\right\|_{0, \hat{\Omega}}^{2}+C_{1}\left\|\widetilde{u}_{z z}\right\|_{0, \hat{\Omega}}^{2}
$$

and

$$
\begin{aligned}
\text { (3.104) } \frac{1}{2} \frac{d}{d t} \int_{\hat{\Omega}} \frac{\widehat{\eta} c_{v}}{\widehat{\Gamma}} \widetilde{\gamma}_{z z}^{2} J d z \leq \varepsilon\left\|\widetilde{\gamma}_{z z t}\right\|_{0, \hat{\Omega}}^{2}+C_{1}\left(\|\widehat{\gamma}\|_{0, \hat{\Omega}}^{2}+\left\|\widehat{\gamma}_{0 z}\right\|_{1, \hat{\Omega}}^{2}\right) \\
+C_{2}\left(\|\widehat{u}\|_{2, \hat{\Omega}}^{2}+\left\|\widehat{\gamma}_{0 t}\right\|_{1, \hat{\Omega}}^{2}+\left\|\widehat{\eta}_{\sigma t}\right\|_{1, \hat{\Omega}}^{2}\right)\|\widehat{\gamma}\|_{3, \hat{\Omega}}^{2},
\end{aligned}
$$

where we have used the relations

$$
\widehat{\eta}_{\sigma t}+\widehat{\eta} \widehat{\nabla} \cdot \widehat{u}=0 \quad \text { and } \quad J_{t}=J \widehat{\nabla} \cdot \widehat{u} .
$$


From (3.97)-(3.104) we obtain

$$
\begin{aligned}
& \frac{1}{2} \frac{d}{d t} \int_{\hat{\Omega}}\left(\widehat{\eta}_{u_{z z}^{2}}^{2}+\frac{p_{\sigma \hat{\eta}}}{\widehat{\eta}} \widetilde{\eta}_{\sigma z z}^{2}+\frac{\widehat{\eta} c_{v}}{\widehat{\Gamma}} \widetilde{\gamma}_{z z}^{2}\right) J d z \\
& +c_{0}\left(\|\widetilde{u}\|_{3, \hat{\Omega}}^{2}+\left\|\widetilde{\bar{\eta}}_{\Omega_{t} z}\right\|_{1, \hat{\Omega}}^{2}+\left\|\widetilde{\gamma}_{z z z}\right\|_{0, \hat{\Omega}}^{2}\right) \\
& \leq(\varepsilon+c d)\left(\|\widetilde{u}\|_{3, \hat{\Omega}}^{2}+\left\|\widehat{\gamma}_{0 z z z}\right\|_{0, \hat{\Omega}}^{2}+\left\|\widehat{\eta}_{\sigma z z}\right\|_{0, \hat{\Omega}}^{2}+\left\|\widehat{u}_{z z t}\right\|_{0, \hat{\Omega}}^{2}+\left\|\widehat{\gamma}_{0 z z t}\right\|_{0, \hat{\Omega}}^{2}\right) \\
& +C_{1}\left(|\widehat{u}|_{2,1, \hat{\Omega}}^{2}+\|\widehat{\gamma}\|_{0, \hat{\Omega}}^{2}+\left\|\widehat{\gamma}_{0 z}\right\|_{1, \hat{\Omega}}^{2}+\left\|\widehat{\gamma}_{0 t}\right\|_{1, \hat{\Omega}}^{2}+\left\|\widehat{\bar{\eta}}_{\Omega_{t}}\right\|_{0, \hat{\Omega}}^{2}\right. \\
& \left.+\left\|\widehat{\eta}_{\sigma z}\right\|_{0, \hat{\Omega}}^{2}+\left\|\vartheta_{0 t}\right\|_{0, \Omega_{t}}^{2}+\|v\|_{1, \Omega_{t}}^{2}+\|\widetilde{g}\|_{1, \hat{\Omega}}^{2}+\|\widetilde{k}\|_{1, \hat{\Omega}}^{2}\right) \\
& +C_{2}\left[\left(X_{3}(\widehat{\Omega})+\int_{0}^{t}\|\widehat{u}\|_{3, \hat{\Omega}}^{2} d t^{\prime}\right)\left(1+X_{3}(\widehat{\Omega})\right) Y_{3}(\widehat{\Omega})\right. \\
& \left.+\left(\left\|\eta_{\sigma}\right\|_{1, \hat{\Omega}}^{2}+\left\|\widehat{\gamma}_{0}\right\|_{1, \hat{\Omega}}^{2}+\|\widehat{\gamma}\|_{3, \hat{\Omega}}^{2}\right)\left(\left\|\vartheta_{0 t}\right\|_{0, \Omega_{t}}^{2}+\|v\|_{1, \Omega_{t}}^{2}\right)+\|\widehat{\gamma}\|_{3, \hat{\Omega}}^{4}\right] .
\end{aligned}
$$

Hence, applying the same argument as in Lemma 3.4 we get (3.88).

LEMma 3.6. Let $v, \varrho, \vartheta_{0}$ be a sufficiently smooth solution of problem (3.3). Then

$$
\begin{aligned}
& \frac{1}{2} \frac{d}{d t} \int_{\Omega_{t}}\left(\varrho v_{x t}^{2}+\frac{p_{\sigma \varrho}}{\varrho} \varrho_{x t}^{2}+\frac{\varrho c_{v}}{\theta} \vartheta_{0 x t}^{2}\right) d x \\
& \quad+c_{0}\left(\left\|v_{t}\right\|_{2, \Omega_{t}}^{2}+\left\|\varrho_{\sigma t}\right\|_{1, \Omega_{t}}^{2}+\left\|\vartheta_{0 x x t}\right\|_{0, \Omega_{t}}^{2}\right) \\
& \leq \varepsilon\left(\left\|v_{x t t}\right\|_{0, \Omega_{t}}^{2}+\left\|\vartheta_{0 x t t t}\right\|_{0, \Omega_{t}}^{2}\right)+C_{1}\left(|v|_{2,0, \Omega_{t}}^{2}+\left\|\varrho_{\sigma x}\right\|_{0, \Omega_{t}}^{2}\right. \\
&+\left\|\varrho_{\sigma t}\right\|_{0, \Omega_{t}}^{2}+\left\|\bar{\varrho}_{\Omega_{t}}\right\|_{0, \Omega_{t}}^{2}+\left\|\vartheta_{0 x}\right\|_{1, \Omega_{t}}^{2}+\left\|\vartheta_{0 t}\right\|_{1, \Omega_{t}}^{2}+\|\vartheta\|_{0, \Omega_{t}}^{2} \\
&\left.+|f|_{1,0, \Omega_{t}}^{2}+|r|_{1,0, \Omega_{t}}^{2}+\left\|\theta_{1 t}\right\|_{2, \Omega_{t}}^{2}+\left\|\theta_{1}\right\|_{1, \Omega_{t}}^{2}\right) \\
&+C_{2}\left(X_{4}+\int_{0}^{t}\|v\|_{4, \Omega_{t^{\prime}}}^{2} d t^{\prime}\right)\left(1+X_{4}\right) Y_{4}
\end{aligned}
$$

where

$$
\begin{aligned}
X_{4} & =|v|_{3,1, \Omega_{t}}^{2}+\left|\varrho_{\sigma}\right|_{2,0, \Omega_{t}}^{2}+\left|\vartheta_{0}\right|_{3,1, \Omega_{t}}^{2}+\left\|\bar{\varrho}_{\Omega_{t}}\right\|_{0, \Omega_{t}}^{2}, \\
Y_{4} & =|v|_{4,2, \Omega_{t}}^{2}+\left|\varrho_{\sigma}\right|_{3,1, \Omega_{t}}^{2}+\left|\vartheta_{0 t}\right|_{3,2, \Omega_{t}}^{2}+\left\|\vartheta_{0 x}\right\|_{3, \Omega_{t}}^{2}+\|\vartheta\|_{0, \Omega_{t}}^{2}+\left\|\bar{\varrho}_{\Omega_{t}}\right\|_{0, \Omega_{t}}^{2} .
\end{aligned}
$$

Pr o of. We use the partition of unity introduced in Lemma 3.4. First we consider interior subdomains. Differentiating $(3.46)_{1}$ with respect to $t$ and 
$\xi$, multiplying the result by $\widetilde{u}_{t \xi} A$ and integrating over $\widetilde{\Omega}$ yields

$$
\begin{aligned}
\frac{1}{2} \frac{d}{d t} \int_{\tilde{\Omega}} \eta \widetilde{u}_{t \xi}^{2} A d \xi & +\frac{1}{2} \mu \int_{\tilde{\Omega}}\left(\nabla_{u_{i}} \widetilde{u}_{j t \xi}+\nabla_{u_{j}} \widetilde{u}_{i t \xi}\right)^{2} A d \xi \\
& +(\nu-\mu)\left\|\nabla_{u} \cdot \widetilde{u}_{t \xi}\right\|_{0, \tilde{\Omega}}^{2}-\int_{\tilde{\Omega}} \widetilde{p}_{\sigma t \xi} \nabla_{u} \cdot \widetilde{u}_{t \xi} A d \xi \\
\leq & \varepsilon\left(\left\|u_{t \xi}\right\|_{1, \tilde{\Omega}}^{2}+\left\|\eta_{\sigma t \xi}\right\|_{0, \tilde{\Omega}}^{2}+\left\|\gamma_{0 t \xi}\right\|_{0, \tilde{\Omega}}^{2}\right) \\
& +C_{1}\left(\left\|u_{t}\right\|_{1, \tilde{\Omega}}^{2}+\left\|\eta_{\sigma \xi}\right\|_{0, \tilde{\Omega}}^{2}+\left\|\eta_{\sigma t}\right\|_{0, \tilde{\Omega}}^{2}+\left\|\bar{\eta}_{\Omega_{t}}\right\|_{0, \tilde{\Omega}}^{2}\right. \\
& \left.+\|\gamma\|_{0, \tilde{\Omega}}^{2}+\left\|\gamma_{0 \xi}\right\|_{0, \tilde{\Omega}}^{2}+\left\|\gamma_{0 t}\right\|_{0, \tilde{\Omega}}^{2}+|\widetilde{g}|_{1,0, \tilde{\Omega}}^{2}\right) \\
& +C_{2}\left(X_{4}(\widetilde{\Omega})+\int_{0}^{t}\|u\|_{4, \tilde{\Omega}}^{2} d t^{\prime}\right)\left(1+X_{4}(\widetilde{\Omega})\right) Y_{4}(\widetilde{\Omega})
\end{aligned}
$$

where

$$
\begin{aligned}
& X_{4}(\widetilde{\Omega})=|u|_{3,1, \tilde{\Omega}}^{2}+\left|\bar{\eta}_{\Omega_{t}}\right|_{2,0, \tilde{\Omega}}^{2}+|\gamma|_{3,1, \tilde{\Omega}}^{2}+\left|\eta_{\sigma}\right|_{2,0, \tilde{\Omega}}^{2}+\left|\gamma_{0}\right|_{3,1, \tilde{\Omega}}^{2} \\
& Y_{4}(\widetilde{\Omega})=|u|_{4,2, \tilde{\Omega}}^{2}+\left|\bar{\eta}_{\Omega_{t}}\right|_{3,1, \tilde{\Omega}}^{2}+|\gamma|_{4,2, \tilde{\Omega}}^{2}+\left|\eta_{\sigma t}\right|_{2,1, \tilde{\Omega}}^{2}+\left|\gamma_{0 t}\right|_{3,2, \tilde{\Omega}}^{2}
\end{aligned}
$$

Next, dividing $(3.46)_{3}$ by $\Gamma$, differentiating with respect to $t$ and $\xi$, multiplying the result by $\widetilde{\gamma}_{t \xi} A$ and integrating over $\widetilde{\Omega}$ we obtain

$$
\begin{aligned}
& \frac{1}{2} \frac{d}{d t} \int_{\tilde{\Omega}} \frac{\eta c_{v}}{\Gamma} \widetilde{\gamma}_{t \xi}^{2} A d \xi+\int_{\tilde{\Omega}} p_{\sigma \Gamma} \nabla_{u} \cdot \widetilde{u}_{t \xi} \widetilde{\gamma}_{t \xi} A d \xi+\frac{\kappa}{\theta^{*}} \int_{\tilde{\Omega}}\left|\nabla_{u} \widetilde{\gamma}_{t \xi}\right|^{2} A d \xi \\
& \leq \varepsilon\left(\left\|\widetilde{u}_{t \xi}\right\|_{1, \tilde{\Omega}}^{2}+\left\|\widetilde{\gamma}_{t \xi}\right\|_{1, \tilde{\Omega}}^{2}+\left\|\vartheta_{0 x t t}\right\|_{0, \Omega_{t}}^{2}\right) \\
&+C_{1}\left(\left\|u_{t}\right\|_{1, \tilde{\Omega}}^{2}+\left\|\gamma_{0 t}\right\|_{1, \tilde{\Omega}}^{2}+\left\|\eta_{\sigma \xi}\right\|_{0, \tilde{\Omega}}^{2}+\left\|\eta_{\sigma t}\right\|_{0, \tilde{\Omega}}^{2}\right. \\
&+\left\|\bar{\eta}_{\Omega_{t}}\right\|_{0, \tilde{\Omega}}^{2}+\left\|\gamma_{0 \xi}\right\|_{0, \tilde{\Omega}}^{2}+\|\gamma\|_{0, \tilde{\Omega}}^{2}+\left\|\vartheta_{0 t}\right\|_{1, \Omega_{t}}^{2}+\left\|v_{t}\right\|_{1, \Omega_{t}}^{2} \\
&\left.+\|v\|_{1, \Omega_{t}}^{2}+\left\|r_{t}\right\|_{0, \Omega_{t}}^{2}+\|r\|_{0, \Omega_{t}}^{2}+\left\|\theta_{1 t}\right\|_{1, \Omega_{t}}^{2}+|\widetilde{k}|_{1,0, \tilde{\Omega}}^{2}\right) \\
&+C_{2}\left(X_{4}+\int_{0}^{t}\|v\|_{4, \Omega_{t^{\prime}}}^{2} d t^{\prime}\right)\left(1+X_{4}\right) Y_{4},
\end{aligned}
$$

where to estimate $\int_{\tilde{\Omega}}\left(\frac{\eta c_{v}}{\Gamma} \zeta \partial_{t} \theta_{\Omega_{t}}\right)_{, t \xi} \widetilde{\gamma}_{t \xi} A d \xi$ we have used

$$
\begin{aligned}
\left\|\partial_{t}^{2} \theta_{\Omega_{t}}\right\|_{0, \tilde{\Omega}}^{2} \leq & \varepsilon\left\|\vartheta_{0 x t t}\right\|_{0, \Omega_{t}}^{2}+C_{1}\left(\left\|v_{t}\right\|_{1, \Omega_{t}}^{2}+\|v\|_{1, \Omega_{t}}^{2}\right. \\
& \left.+\left\|\vartheta_{0 t}\right\|_{1, \Omega_{t}}^{2}+\left\|r_{t}\right\|_{0, \Omega_{t}}^{2}+\|r\|_{0, \Omega_{t}}^{2}+\left\|\theta_{1 t}\right\|_{1, \Omega_{t}}^{2}\right) \\
& +C_{2}\left(X_{4}+\int_{0}^{t}\|v\|_{4, \Omega_{t^{\prime}}}^{2} d t^{\prime}\right)\left(1+X_{4}\right) Y_{4} .
\end{aligned}
$$


Since

$$
\begin{aligned}
\widetilde{p}_{\sigma t \xi}= & \widetilde{p}_{\sigma \eta \eta} \eta_{\sigma \xi} \eta_{\sigma t}+\widetilde{p}_{\sigma \eta \Gamma}\left(\eta_{\sigma \xi} \gamma_{0 \xi}+\eta_{\sigma t} \gamma_{0 t}\right)+\widetilde{p}_{\sigma \Gamma \Gamma} \gamma_{0 \xi} \gamma_{0 t} \\
& +p_{\sigma \eta} \overline{\bar{\eta}}_{\Omega_{t} \xi t}+p_{\sigma \Gamma} \widetilde{\gamma}_{\xi t}-\left(p_{\sigma \eta} \eta_{\sigma}+p_{\sigma \Gamma} \gamma\right) \zeta_{\xi t}-p_{\sigma \eta}\left(\zeta_{t} \eta_{\sigma \xi}+\zeta_{\xi} \eta_{\sigma t}\right) \\
& -p_{\sigma \Gamma}\left(\zeta_{t} \gamma_{0 \xi}+\zeta_{\xi} \gamma_{0 t}\right)
\end{aligned}
$$

using (3.107), (3.108), equation (3.52), (3.109) and Lemma 5.1 of [21] with $G=\widetilde{\Omega}, v=\widetilde{u}_{t \xi}$ we get

$$
\begin{aligned}
(3.110) \quad \frac{1}{2} \frac{d}{d t} \int_{\tilde{\Omega}} & \left(\eta \widetilde{u}_{t \xi}^{2}+\frac{p_{\sigma \eta}}{\eta} \widetilde{\eta}_{\Omega_{t} t \xi}^{2}+\frac{\eta c_{v}}{\Gamma} \widetilde{\gamma}_{t \xi}^{2}\right) A d \xi \\
& +c_{0}\left(\left\|\widetilde{u}_{t}\right\|_{2, \tilde{\Omega}}^{2}+\left\|\widetilde{\gamma}_{t \xi \xi}\right\|_{0, \tilde{\Omega}}^{2}+\left\|\widetilde{\bar{\eta}}_{\Omega_{t} t \xi}\right\|_{0, \tilde{\Omega}}^{2}\right) \\
\leq & \varepsilon\left(\left\|u_{t \xi}\right\|_{1, \tilde{\Omega}}^{2}+\left\|\eta_{\sigma t \xi}\right\|_{0, \tilde{\Omega}}^{2}+\left\|\gamma_{0 t \xi \xi}\right\|_{0, \tilde{\Omega}}^{2}+\left\|\vartheta_{0 x t t}\right\|_{0, \Omega_{t}}^{2}\right) \\
& +C_{1}\left(|u|_{2,0, \tilde{\Omega}}^{2}+\left\|\eta_{\sigma \xi}\right\|_{0, \tilde{\Omega}}^{2}+\left\|\eta_{\sigma t}\right\|_{0, \tilde{\Omega}}^{2}+\left\|\bar{\eta}_{\Omega_{t}}\right\|_{0, \tilde{\Omega}}^{2}+\left\|\gamma_{0 \xi}\right\|_{1, \tilde{\Omega}}^{2}\right. \\
& +\left\|\gamma_{0 t}\right\|_{1, \tilde{\Omega}}^{2}+\|\gamma\|_{0, \tilde{\Omega}}^{2}+\left\|v_{t}\right\|_{1, \Omega_{t}}^{2}+\|v\|_{1, \Omega_{t}}^{2}+\left\|\vartheta_{0 t}\right\|_{1, \Omega_{t}}^{2} \\
& \left.+|\widetilde{g}|_{1,0, \tilde{\Omega}}^{2}+|\widetilde{k}|_{1,0, \tilde{\Omega}}^{2}+\left\|r_{t}\right\|_{0, \Omega_{t}}^{2}+\|r\|_{0, \Omega_{t}}^{2}+\left\|\theta_{1 t}\right\|_{1, \Omega_{t}}^{2}\right) \\
& +C_{2}\left(X_{4}+\int_{0}^{t}\|v\|_{4, \Omega_{t^{\prime}}}^{2} d t^{\prime}\right)\left(1+X_{4}\right) Y_{4},
\end{aligned}
$$

where we have also used the following estimate for a solution $\widetilde{u}, \eta_{\sigma}$ of the Stokes problem (3.57):

$$
\begin{aligned}
\left\|\widetilde{u}_{t}\right\|_{2, \tilde{\Omega}}^{2}+\left\|\widetilde{\bar{\eta}}_{\Omega_{t} t \xi}\right\|_{0, \tilde{\Omega}}^{2} \leq & C_{1}\left(\left\|\widetilde{u}_{t t}\right\|_{0, \tilde{\Omega}}^{2}+|u|_{2,1, \tilde{\Omega}}^{2}+\left|\eta_{\sigma}\right|_{1,0, \tilde{\Omega}}^{2}+\left\|\gamma_{0 \xi}\right\|_{1 \tilde{\Omega}}^{2}\right. \\
& \left.+\left\|\gamma_{0 t}\right\|_{1, \tilde{\Omega}}^{2}+\|\gamma\|_{0, \tilde{\Omega}}^{2}+|\widetilde{g}|_{1,0, \tilde{\Omega}}^{2}\right) \\
& +C_{2}\left(X_{4}+\int_{0}^{t}\|v\|_{4, \Omega_{t}}^{2} d t^{\prime}\right) Y_{4}+c\left\|\left(\nabla_{u} \cdot \widetilde{u}\right)_{, t}\right\|_{1, \tilde{\Omega}}^{2}
\end{aligned}
$$

For subdomains near the boundary we obtain the inequality

$$
\begin{aligned}
& \frac{1}{2} \frac{d}{d t} \int_{\hat{\Omega}}\left(\widehat{\eta} \widetilde{u}_{t \tau}^{2}+\frac{p_{\sigma \hat{\eta}}}{\widehat{\eta}} \widetilde{\bar{\eta}}_{\Omega_{t} t \tau}^{2}+\frac{\widehat{\eta} c_{v}}{\widehat{\Gamma}} \widetilde{\gamma}_{t \tau}^{2}\right) J d z \\
& \quad+c_{0}\left(\left\|\widetilde{u}_{\tau \tau}\right\|_{1, \hat{\Omega}}^{2}+\left\|\widetilde{\gamma}_{t \tau z}\right\|_{0, \hat{\Omega}}^{2}+\left\|\widetilde{\bar{\eta}}_{\Omega_{t} t \tau}\right\|_{0, \tilde{\Omega}}^{2}\right) \\
& \leq \varepsilon\left(\left\|\widetilde{u}_{t z}\right\|_{1, \hat{\Omega}}^{2}+\left\|\widehat{\eta}_{\sigma t z}\right\|_{0, \hat{\Omega}}^{2}+\left\|\widetilde{\gamma}_{t z}\right\|_{1, \hat{\Omega}}^{2}+\left\|\vartheta_{0 x t t}\right\|_{0, \Omega_{t}}^{2}\right) \\
& \quad+C_{1}\left(|\widehat{u}|_{2,0, \hat{\Omega}}^{2}+\left\|\widehat{\eta}_{\sigma z}\right\|_{0, \hat{\Omega}}^{2}+\left\|\widehat{\eta}_{\sigma t}\right\|_{0, \hat{\Omega}}^{2}+\left\|\widehat{\bar{\eta}}_{\Omega_{t}}\right\|_{0, \hat{\Omega}}^{2}+\left\|\widehat{\gamma}_{0 z}\right\|_{1, \hat{\Omega}}^{2}\right. \\
& \quad+\left\|\widehat{\gamma}_{0 t}\right\|_{1, \hat{\Omega}}^{2}+\|\widehat{\gamma}\|_{0, \hat{\Omega}}^{2}+\left\|v_{t}\right\|_{1, \Omega_{t}}^{2}+\|v\|_{1, \Omega_{t}}^{2}+\left\|\vartheta_{0 t}\right\|_{1, \Omega_{t}}^{2}+|\widetilde{g}|_{1,0, \hat{\Omega}}^{2}
\end{aligned}
$$




$$
\begin{aligned}
& \left.+\mid \widetilde{k}_{1,0, \hat{\Omega}^{2}}^{2}+\left\|r_{t}\right\|_{0, \Omega_{t}}^{2}+\|r\|_{0, \Omega_{t}}^{2}+\left\|\vartheta_{1 t}\right\|_{1, \Omega_{t}}^{2}+\left\|\widetilde{\Gamma}_{1 t}\right\|_{2, \hat{\Omega}^{2}}^{2}+\left\|\widetilde{\Gamma}_{1}\right\|_{1, \hat{\Omega}}^{2}\right) \\
& +C_{2}\left(X_{4}+\int_{0}^{t}\|v\|_{4, \Omega_{t^{\prime}}}^{2} d t^{\prime}\right)\left(1+X_{4}\right) Y_{4}
\end{aligned}
$$

where we have used the following estimates:

$$
\begin{aligned}
& \int_{\hat{S}}\left|\left(\widehat{\mathbb{T}}\left(\widetilde{u}, \widetilde{p}_{\sigma}\right) \widehat{n}\right)_{, t \tau} \widetilde{u}_{t \tau} J\right| d z^{\prime} \\
& \leq \varepsilon\left(\left\|\widehat{u}_{t \tau}\right\|_{1, \hat{\Omega}}^{2}+\left\|\widehat{u}_{t z z}\right\|_{0, \hat{\Omega}}^{2}\right)+C_{1}|\widehat{u}|_{2,1, \hat{\Omega}}^{2} \\
&+C_{2}\left[\|\widehat{u}\|_{2, \hat{\Omega}}^{4}+|\widehat{u}|_{3,2, \hat{\Omega}}^{2}\left\|\int_{0}^{t} \widehat{u} d t^{\prime}\right\|_{3, \hat{\Omega}}^{2}\left(1+\|\widehat{u}\|_{3, \hat{\Omega}}^{2}\right)\right]
\end{aligned}
$$

and

$$
\begin{aligned}
& \int_{\hat{S}}\left|\left(\widehat{n} \cdot \widehat{\Gamma}^{-1} \widehat{\nabla} \widetilde{\gamma}\right)_{, t \tau} \widetilde{\gamma}_{t \tau} J\right| d z^{\prime} \\
& \leq \varepsilon\left(\left\|\widetilde{\gamma}_{t \tau}\right\|_{1, \hat{\Omega}}^{2}+\left\|\widetilde{\gamma}_{t z z}\right\|_{0, \hat{\Omega}}^{2}\right) \\
&+C_{1}\left(\left\|\widehat{\gamma}_{0 t}\right\|_{1, \hat{\Omega}}^{2}+\|v\|_{2, \Omega_{t}}^{2}+\left\|\vartheta_{0 t}\right\|_{1, \Omega_{t}}^{2}+\left\|\widetilde{\Gamma}_{1 t}\right\|_{2, \hat{\Omega}}^{2}+\left\|\widetilde{\Gamma}_{1}\right\|_{1, \hat{\Omega}}^{2}\right) \\
&+C_{2}\left[\left(X_{4}(\widehat{\Omega})+\int_{0}^{t}\|\widehat{u}\|_{4, \Omega_{t^{\prime}}}^{2} d t^{\prime}\right)\left(1+X_{4}(\widehat{\Omega})\right) Y_{4}(\widehat{\Omega})\right. \\
&\left.+\int_{0}^{t}\|\widehat{u}\|_{4, \hat{\Omega}}^{2} d t^{\prime}\left(\left\|\vartheta_{0 t}\right\|_{0, \Omega_{t}}^{2}+\|v\|_{1, \Omega_{t}}^{2}\right)\right] .
\end{aligned}
$$

Next, differentiating the third component of (3.63) with respect to $t$, multiplying the result by $\widetilde{\bar{\eta}}_{\Omega_{t} n} J$ and integrating over $\widehat{\Omega}$ yields

$$
\begin{aligned}
& \frac{1}{2} \frac{d}{d t} \int_{\hat{\Omega}} \frac{p_{\sigma \hat{\eta}}}{\widehat{\eta}}(\nu+\mu) \widetilde{\bar{\eta}}_{\Omega_{t} n t}^{2} J d z+c_{0}\left\|\widetilde{\bar{\eta}}_{\Omega_{t} n t}\right\|_{0, \hat{\Omega}}^{2} \\
& \leq(\varepsilon+c d)\left(\left\|\widetilde{u}_{z z t}\right\|_{0, \hat{\Omega}}^{2}+\left\|\widetilde{\bar{\eta}}_{\Omega_{t} z t}\right\|_{0, \hat{\Omega}}^{2}\right)+\varepsilon\left\|\vartheta_{0 x t t}\right\|_{0, \Omega_{t}}^{2} \\
&+C_{1}\left(\left\|\widetilde{u}_{t \tau}\right\|_{0, \hat{\Omega}}^{2}+|\widehat{u}|_{2,0, \hat{\Omega}}^{2}+\left\|\widehat{\eta}_{\sigma t}\right\|_{0, \hat{\Omega}}^{2}\right. \\
&+\left\|\widehat{\bar{\eta}}_{\Omega_{t}}\right\|_{0, \hat{\Omega}}^{2}+\left\|\widehat{\gamma}_{0 t}\right\|_{1, \hat{\Omega}}^{2}+\left\|v_{t}\right\|_{1, \Omega_{t}}^{2}+\left\|\vartheta_{0 t}\right\|_{1, \Omega_{t}}^{2} \\
&\left.+\left\|r_{t}\right\|_{0, \Omega_{t}}^{2}+\|r\|_{0, \Omega_{t}}^{2}+\left\|\theta_{1 t}\right\|_{1, \Omega_{t}}^{2}+|\widetilde{g}|_{1,0, \hat{\Omega}}^{2}\right) \\
&+C_{2}\left(X_{4}+\int_{0}^{t}\|v\|_{4, \Omega_{t^{\prime}}}^{2} d t^{\prime}\right)\left(1+X_{4}\right) Y_{4},
\end{aligned}
$$

where we have used (3.109). 
Differentiating the third component of (3.66) with respect to $t$, multiplying the result by $\widetilde{u}_{3 n n t} J$ and integrating over $\widehat{\Omega}$ implies

$$
\begin{aligned}
& \frac{1}{2} \frac{d}{d t} \int_{\hat{\Omega}} \hat{\eta}\left|\widetilde{u}_{3 n t}\right|^{2} J d z+c_{0}\left\|\widetilde{u}_{3 n n t}\right\|_{0, \hat{\Omega}}^{2} \\
& \leq(\varepsilon+c d)\left(\left\|\widetilde{u}_{z z t}\right\|_{0, \hat{\Omega}}^{2}+\left\|\widetilde{\bar{\eta}}_{\Omega_{t} z t}\right\|_{0, \hat{\Omega}}^{2}\right)+\varepsilon\left\|\widetilde{u}_{z t t}\right\|_{0, \hat{\Omega}}^{2} \\
&+C_{1}\left(\left\|\widetilde{u}_{z \tau t}\right\|_{0, \hat{\Omega}}^{2}+\left\|\widetilde{\bar{\eta}}_{\Omega_{t} n t}\right\|_{0, \hat{\Omega}}^{2}+|\widehat{u}|_{2,0, \hat{\Omega}}^{2}\right. \\
&+\left\|\widehat{\eta}_{\sigma t}\right\|_{0, \hat{\Omega}}^{2}+\left\|\widehat{\bar{\eta}}_{\Omega_{t}}\right\|_{0, \hat{\Omega}}^{2}+\left\|\widehat{\eta}_{\sigma z}\right\|_{0, \hat{\Omega}}^{2}+\left\|\widehat{\gamma}_{0 t}\right\|_{1, \hat{\Omega}}^{2} \\
&\left.+\left\|\widehat{\gamma}_{0 z}\right\|_{1, \hat{\Omega}}^{2}+\|\widehat{\gamma}\|_{0, \hat{\Omega}}^{2}+|\widetilde{g}|_{1,0, \hat{\Omega}}^{2}+|\widetilde{k}|_{1,0, \hat{\Omega}}^{2}\right) \\
&+C_{2}\left(X_{4}(\widehat{\Omega})+\int_{0}^{t}\|\widehat{u}\|_{4, \hat{\Omega}}^{2} d t^{\prime}\right)\left(1+X_{4}(\widehat{\Omega})\right) Y_{4}(\widehat{\Omega}) .
\end{aligned}
$$

Differentiating (3.68) with respect to $t$ and $\tau$, multiplying by $\widetilde{u}_{t \tau}^{\prime} J$, integrating over $\widehat{\Omega}$ and using (3.69) gives

$$
\begin{aligned}
\left\|\widetilde{u}_{z t \tau}^{\prime}\right\|_{0, \hat{\Omega}}^{2} & +\left\|\widetilde{\bar{\eta}}_{\Omega_{t} t \tau}\right\|_{0, \hat{\Omega}}^{2} \\
\leq & (\varepsilon+c d)\left(\left\|\widetilde{u}_{z z t}\right\|_{0, \hat{\Omega}}^{2}+\left\|\widehat{\eta}_{\sigma z t}\right\|_{0, \hat{\Omega}}^{2}\right) \\
& +C_{1}\left(\left\|\left(\operatorname{div} \widetilde{u}^{\prime}\right)_{, \tau t}\right\|_{0, \hat{\Omega}}^{2}+|\widehat{u}|_{2,0, \hat{\Omega}}^{2}+\left\|\widehat{\eta}_{\sigma t}\right\|_{0, \hat{\Omega}}^{2}+\left\|\widehat{\eta}_{\sigma z}\right\|_{0, \hat{\Omega}}^{2}\right. \\
& \left.+\left\|\widehat{\bar{\eta}}_{\Omega_{t}}\right\|_{0, \hat{\Omega}}^{2}+\left\|\widehat{\gamma}_{0 z}\right\|_{1, \hat{\Omega}}^{2}+\left\|\widehat{\gamma}_{0 t}\right\|_{1, \hat{\Omega}}^{2}+\|\widehat{\gamma}\|_{0, \hat{\Omega}}^{2}+|\widetilde{g}|_{1,0, \hat{\Omega}}^{2}\right) \\
& +C_{2}\left(X_{4}(\widehat{\Omega})+\int_{0}^{t}\|\widehat{u}\|_{4, \hat{\Omega}}^{2} d t^{\prime}\right)\left(1+X_{4}(\widehat{\Omega})\right) Y_{4}(\widehat{\Omega}) .
\end{aligned}
$$

Moreover, from (3.68) we get

$$
\begin{aligned}
& \left\|\widetilde{u}_{n n t}^{\prime}\right\|_{0, \hat{\Omega}}^{2} \leq(\varepsilon+c d)\left(\left\|\widetilde{u}_{z z t}\right\|_{0, \hat{\Omega}}^{2}+\left\|\widehat{\eta}_{\sigma z t}\right\|_{0, \hat{\Omega}}^{2}\right) \\
& \quad+C_{1}\left(\left\|\widetilde{u}_{z t \tau}\right\|_{0, \hat{\Omega}}^{2}+\left\|\bar{\eta}_{\Omega_{t} t \tau}\right\|_{0, \hat{\Omega}}^{2}+|\widehat{u}|_{2,0, \hat{\Omega}}^{2}+\left\|\widehat{\eta}_{\sigma z}\right\|_{0, \hat{\Omega}}^{2}+\left\|\widehat{\eta}_{\sigma t}\right\|_{0, \hat{\Omega}}^{2}\right. \\
& \left.\quad+\left\|\widehat{\bar{\eta}}_{\Omega_{t}}\right\|_{0, \hat{\Omega}}+\left\|\widehat{\gamma}_{0 z}\right\|_{1, \hat{\Omega}}^{2}+\left\|\widehat{\gamma}_{0 t}\right\|_{1, \hat{\Omega}}^{2}+\|\widehat{\gamma}\|_{0, \hat{\Omega}}^{2}+|\widetilde{g}|_{1,0, \hat{\Omega}}^{2}\right) \\
& \quad+C_{2}\left(X_{4}(\widehat{\Omega})+\int_{0}^{t}\|\widehat{u}\|_{0, \hat{\Omega}}^{2} d t^{\prime}\right)\left(1+X_{4}(\widehat{\Omega})\right) Y_{4}(\widehat{\Omega}) .
\end{aligned}
$$

Next, we have

$$
\frac{d}{d t} \int_{\hat{\Omega}} \widehat{\eta}_{z t}^{2} J d z \leq \varepsilon\left\|\widetilde{u}_{z t t}\right\|_{0, \hat{\Omega}}^{2}+C_{1}\left\|\widetilde{u}_{t}\right\|_{0, \hat{\Omega}}^{2} .
$$


Finally, by using (3.109) we obtain

$$
\begin{aligned}
& \frac{1}{2} \frac{d}{d t} \int_{\hat{\Omega}} \frac{\widehat{\eta} c_{v}}{\widehat{\Gamma}} \widetilde{\gamma}_{n t}^{2} J d z+\frac{\kappa}{\theta^{*}}\left\|\widetilde{\gamma}_{n n t}\right\|_{0, \hat{\Omega}}^{2} \\
& \leq \quad(\varepsilon+c d)\left(\left\|\widetilde{\gamma}_{z z t}\right\|_{0, \hat{\Omega}}^{2}+\left\|\widetilde{\bar{\eta}}_{\Omega_{t} z t}\right\|_{0, \hat{\Omega}}^{2}\right)+\varepsilon\left(\left\|\widehat{\gamma}_{0 z t t}\right\|_{0, \hat{\Omega}}^{2}+\left\|\vartheta_{0 x t t}\right\|_{0, \Omega_{t}}^{2}\right) \\
& \quad+C_{1}\left(\left\|\widetilde{\gamma}_{t \tau}\right\|_{1, \hat{\Omega}}^{2}+|\widehat{u}|_{2,1, \hat{\Omega}}^{2}+\left\|\widehat{\eta}_{\sigma z}\right\|_{0, \hat{\Omega}}^{2}+\left\|\widehat{\eta}_{\sigma t}\right\|_{0, \hat{\Omega}}^{2}\right. \\
& \quad+\left\|\widehat{\bar{\eta}}_{\Omega_{t}}\right\|_{0, \hat{\Omega}}^{2}+\left\|\widehat{\gamma}_{0 z}\right\|_{1, \hat{\Omega}}^{2}+\left\|\widehat{\gamma}_{0 t}\right\|_{1, \hat{\Omega}}^{2}+\|\widehat{\gamma}\|_{0, \hat{\Omega}}^{2} \\
& \left.\quad+\left\|v_{t}\right\|_{1, \Omega_{t}}^{2}+\left\|\vartheta_{0 t}\right\|_{1, \Omega_{t}}^{2}+\left\|r_{t}\right\|_{0, \Omega_{t}}^{2}+\|r\|_{0, \Omega_{t}}^{2}\right) \\
& \quad+C_{2}\left(X_{4}+\int_{0}^{t}\|v\|_{4, \Omega_{t^{\prime}}}^{2} d t^{\prime}\right)\left(1+X_{4}\right) Y_{4}
\end{aligned}
$$

Taking into account inequalities (3.111)-(3.117) we get

$$
\frac{1}{2} \frac{d}{d t} \int_{\hat{\Omega}}\left(\widehat{\eta} \widetilde{u}_{z t}^{2}+\frac{p_{\sigma \hat{\eta}}}{\widehat{\eta}} \widetilde{\bar{\eta}}_{\Omega_{t} z t}^{2}+\frac{\widehat{\eta} c_{v}}{\widehat{\Gamma}} \widetilde{\gamma}_{z t}^{2}\right) J d z
$$

$$
+c_{0}\left(\left\|\widetilde{u}_{t z}\right\|_{1, \hat{\Omega}}^{2}+\left\|\widetilde{\gamma}_{t z z}\right\|_{0, \hat{\Omega}}^{2}+\left\|\widetilde{\bar{\eta}}_{\Omega_{t} z t}\right\|_{0, \hat{\Omega}}^{2}\right)
$$$$
\leq \varepsilon\left(\left\|\widetilde{u}_{t z}\right\|_{1, \hat{\Omega}}^{2}+\left\|\widehat{\eta}_{\sigma z t}\right\|_{0, \hat{\Omega}}^{2}+\left\|\widetilde{\gamma}_{t z}\right\|_{1, \hat{\Omega}}^{2}+\left\|\widetilde{u}_{z t t}\right\|_{0, \hat{\Omega}}^{2}+\left\|\widetilde{\gamma}_{z t t}\right\|_{0, \hat{\Omega}}^{2}+\left\|\vartheta_{0 x t t}\right\|_{0, \Omega_{t}}^{2}\right)
$$$$
+(\varepsilon+c d)\left(\left\|\widetilde{\gamma}_{z z t}\right\|_{0, \hat{\Omega}}^{2}+\left\|\widetilde{\bar{\eta}}_{\Omega_{t} z t}\right\|_{0, \hat{\Omega}}^{2}+\left\|\widetilde{u}_{z z t}\right\|_{0, \hat{\Omega}}^{2}+\left\|\widehat{\eta}_{\sigma z t}\right\|_{0, \hat{\Omega}}^{2}\right)
$$$$
+C_{1}\left(|\widehat{u}|_{2,0, \hat{\Omega}}^{2}+\left\|\widehat{\eta}_{\sigma z}\right\|_{0, \hat{\Omega}}^{2}+\left\|\widehat{\eta}_{\sigma t}\right\|_{0, \hat{\Omega}}^{2}+\left\|\widehat{\bar{\eta}}_{\Omega_{t}}\right\|_{0, \hat{\Omega}}^{2}+\left\|\widehat{\gamma}_{0 z}\right\|_{1, \hat{\Omega}}^{2}\right.
$$$$
+\left\|\widehat{\gamma}_{0 t}\right\|_{1, \hat{\Omega}}^{2}+\|\widehat{\gamma}\|_{0, \hat{\Omega}}^{2}+\left\|\widetilde{u}_{t \tau}\right\|_{1, \hat{\Omega}}^{2}+\left\|v_{t}\right\|_{1, \Omega_{t}}^{2}+\left\|\vartheta_{0 t}\right\|_{1, \Omega_{t}}^{2}
$$$$
+\|v\|_{1, \Omega_{t}}^{2}+|\widetilde{g}|_{1,0, \hat{\Omega}}^{2}+|\widetilde{k}|_{1,0, \hat{\Omega}}^{2}+\left\|\widetilde{\Gamma}_{1 t}\right\|_{2, \hat{\Omega}}^{2}
$$$$
\left.+\left\|\widetilde{\Gamma}_{1}\right\|_{1, \hat{\Omega}}^{2}+\left\|\theta_{1 t}\right\|_{1, \Omega_{t}}^{2}+\left\|r_{t}\right\|_{0, \Omega_{t}}^{2}+\|r\|_{0, \Omega_{t}}^{2}\right)
$$$$
+C_{2}\left(X_{4}+\int_{0}^{t}\|v\|_{4, \Omega_{t^{\prime}}}^{2} d t^{\prime}\right)\left(1+X_{4}\right) Y_{4} \text {. }
$$

Inequalities (3.110) and (3.118) yield the assertion of the lemma.

LEMMA 3.7. Let $v, \varrho, \vartheta_{0}$ be a sufficiently smooth solution of problem (3.3). Then

(3.119) $\quad \frac{1}{2} \frac{d}{d t} \int_{\Omega_{t}}\left(\varrho v_{t t}^{2}+\frac{p_{\sigma \varrho}}{\varrho} \varrho_{\sigma t t}^{2}+\frac{\varrho c_{v}}{\theta} \vartheta_{0 t t}^{2}\right) d x$

$$
+c_{0}\left(\left\|v_{t t}\right\|_{1, \Omega_{t}}^{2}+\left\|\varrho_{\sigma t t}\right\|_{0, \Omega_{t}}^{2}+\left\|\vartheta_{0 t t}\right\|_{1, \Omega_{t}}^{2}\right)
$$




$$
\begin{aligned}
\leq & C_{1}\left(\left\|v_{t}\right\|_{1, \Omega_{t}}^{2}+\left\|\vartheta_{0 t}\right\|_{1, \Omega_{t}}^{2}+|f|_{1,0, \Omega_{t}}^{2}\right. \\
& \left.+\left\|f_{t t}\right\|_{0, \Omega_{t}}^{2}+|r|_{1,0, \Omega_{t}}^{2}+\left\|r_{t t}\right\|_{0, \Omega_{t}}^{2}+\left|\theta_{1}\right|_{3,1, \Omega_{t}}^{2}\right) \\
& +C_{2} X_{5}\left(1+X_{5}\right) Y_{5},
\end{aligned}
$$

where

$X_{5}=|v|_{3,1, \Omega_{t}}^{2}+\left|\varrho_{\sigma}\right|_{2,0, \Omega_{t}}^{2}+\left|\vartheta_{0}\right|_{3,1, \Omega_{t}}^{2}, \quad Y_{5}=|v|_{4,2, \Omega_{t}}^{2}+\left|\varrho_{\sigma}\right|_{3,1, \Omega_{t}}^{2}+\left|\vartheta_{0}\right|_{4,2, \Omega_{t}}^{2}$.

Proof. Differentiating (3.3) 1 twice with respect to $t$, multiplying by $v_{t t}$ and integrating over $\Omega_{t}$ yields

$$
\frac{1}{2} \frac{d}{d t} \int_{\Omega_{t}} \varrho v_{t t}^{2} d x+\frac{\mu}{2} E_{\Omega_{t}}\left(v_{t t}\right)+(\nu-\mu)\left\|\operatorname{div} v_{t t}\right\|_{0, \Omega_{t}}^{2}
$$

$-\int_{\Omega_{t}} p_{\sigma \varrho} \varrho_{\sigma t t} \operatorname{div} v_{t t} d x-\int_{\Omega_{t}} p_{\sigma \theta} \vartheta_{0 t t} \operatorname{div} v_{t t} d x-\int_{S_{t}}\left(n_{i} T^{i j}\left(v, p_{\sigma}\right)\right)_{, t t} v_{i t t} d s$

$$
\leq \varepsilon\left(\left\|v_{t t}\right\|_{1, \Omega_{t}}^{2}+\left\|\varrho_{\sigma t t}\right\|_{0, \Omega_{t}}^{2}\right)+C_{1}\left(|f|_{1,0, \Omega_{t}}^{2}+\left\|f_{t t}\right\|_{0, \Omega_{t}}^{2}\right)+C_{2} X_{5}\left(1+X_{5}\right) Y_{5} .
$$

Next, dividing (3.3) $)_{3}$ by $\theta$, differentiating twice with respect to $t$, multiplying by $\vartheta_{0 t t}$ and integrating over $\Omega_{t}$ yields

$$
\begin{aligned}
& \frac{1}{2} \frac{d}{d t} \int_{\Omega_{t}} \frac{\varrho c_{v}}{\theta} \vartheta_{0 t t} d x+\frac{\kappa}{\theta^{*}} \int_{\Omega_{t}}\left|\nabla \vartheta_{0 t t}\right|^{2} d x \\
& \quad+\int_{\Omega_{t}} p_{\sigma \theta} \vartheta_{0 t t} \operatorname{div} v_{t t} d x-\int_{S_{t}}\left(\frac{n \cdot \nabla \vartheta_{0}}{\theta}\right)_{, t t} \vartheta_{0 t t} d s \\
& \leq \varepsilon\left(\left\|v_{t t}\right\|_{1, \Omega_{t}}^{2}+\left\|\varrho_{\sigma t t}\right\|_{0, \Omega_{t}}^{2}+\left\|\vartheta_{0 t t}\right\|_{1, \Omega_{t}}^{2}\right) \\
& \quad+C_{1}\left(|r|_{1,0, \Omega_{t}}^{2}+\left\|r_{t t}\right\|_{0, \Omega_{t}}^{2}+\left\|\vartheta_{1 t t}\right\|_{1, \Omega_{t}}^{2}\right)+C_{2} X_{5}\left(1+X_{5}\right) Y_{5} .
\end{aligned}
$$

Moreover, we have

$$
\left\|\varrho_{\sigma t t}\right\|_{0, \Omega_{t}}^{2} \leq c\left\|v_{t}\right\|_{1, \Omega_{t}}^{2}+C_{2} X_{5}\left(1+X_{5}\right) Y_{5}
$$

and

$$
\begin{aligned}
\left\|\vartheta_{0 t t}\right\|_{0, \Omega_{t}}^{2} \leq & \varepsilon\left\|\vartheta_{0 x t t}\right\|_{0, \Omega_{t}}^{2}+C_{1}\left(\left\|v_{t}\right\|_{1, \Omega_{t}}^{2}+\left\|\vartheta_{0 t}\right\|_{1, \Omega_{t}}^{2}+\left\|r_{t}\right\|_{0, \Omega_{t}}^{2}\right. \\
& \left.+\|r\|_{0, \Omega_{t}}^{2}+\left\|\theta_{1 t}\right\|_{1, \Omega_{t}}^{2}\right) \\
& +C_{2}\left(X_{5}+\int_{0}^{t}\|v\|_{3, \Omega_{t^{\prime}}}^{2} d t^{\prime}\right)\left(1+X_{5}\right) Y_{5}
\end{aligned}
$$

where we have used the continuity equation $(3.3)_{1}$ and energy equation $(3.3)_{2}$, respectively.

From (3.120)-(3.123), using the continuity equation $(3.3)_{2}$ and Lemma 5.4 of [21] we get (3.119). 
Summarizing, from Lemmas 3.5-3.7 we obtain

LEMMA 3.8. Let $v, \varrho, \vartheta_{0}$ be a sufficiently smooth solution of problem (3.3). Then

$$
\begin{aligned}
& \quad \frac{1}{2} \frac{d}{d t} \int_{\Omega_{t}}\left(\varrho\left|D_{x, t}^{2} v\right|^{2}+\frac{p_{\sigma \varrho}}{\varrho}\left|D_{x, t}^{2} \varrho_{\sigma}\right|^{2}+\frac{\varrho c_{v}}{\theta}\left|D_{x, t}^{2} \vartheta_{0}\right|^{2}\right) d x \\
& \quad+c_{0}\left(|v|_{3,1, \Omega_{t}}^{2}+\left|\varrho_{\sigma t}\right|_{1,0, \Omega_{t}}^{2}+\left\|\varrho_{\sigma x}\right\|_{0, \Omega_{t}}^{2}+\left|\vartheta_{0 t}\right|_{2,1, \Omega_{t}}^{2}+\left\|\vartheta_{0 x x x}\right\|_{0, \Omega_{t}}^{2}\right) \\
& \leq C_{1}\left(|v|_{2,0, \Omega_{t}}^{2}+\left\|\varrho_{\sigma x}\right\|_{0, \Omega_{t}}^{2}+\left\|\varrho_{\sigma t}\right\|_{0, \Omega_{t}}^{2}+\left\|\varrho_{\Omega_{t}}\right\|_{0, \Omega_{t}}^{2}+\left\|\vartheta_{0 x}\right\|_{1, \Omega_{t}}^{2}\right. \\
& \quad+\left\|\vartheta_{0 t}\right\|_{1, \Omega_{t}}^{2}+\|\vartheta\|_{0, \Omega_{t}}^{2}+|f|_{1,0, \Omega_{t}}^{2}+\left\|f_{t t}\right\|_{0, \Omega_{t}}^{2}+|r|_{1,0, \Omega_{t}}^{2} \\
& \left.\quad+\left\|r_{t t}\right\|_{1,0, \Omega_{t}}^{2}+\|r\|_{0, \Omega_{t}}+\left|\theta_{1}\right|_{3,1, \Omega_{t}}^{2}+\left\|\theta_{1}\right\|_{1, \Omega_{t}}\right) \\
& \quad+C_{2}\left(X_{6}+\int_{0}^{t}\|v\|_{4, \Omega_{t^{\prime}}}^{2} d t^{\prime}\right)\left(1+X_{6}\right) Y_{6},
\end{aligned}
$$

where

$$
\begin{aligned}
X_{6} & =|v|_{3,1, \Omega_{t}}^{2}+\left|\varrho_{\sigma}\right|_{2,0, \Omega_{t}}^{2}+\left|\vartheta_{0}\right|_{3,1, \Omega_{t}}^{2}+\left\|\bar{\varrho}_{\Omega_{t}}\right\|_{0, \Omega_{t}}^{2}, \\
Y_{6} & =|v|_{4,2, \Omega_{t}}^{2}+\left|\varrho_{\sigma}\right|_{3,1, \Omega_{t}}^{2}+\left|\vartheta_{0 t}\right|_{3,2, \Omega_{t}}^{2}+\left\|\vartheta_{0 x}\right\|_{3, \Omega_{t}}^{2}+\|\vartheta\|_{0, \Omega_{t}}^{2}+\left\|\varrho_{\Omega_{t}}\right\|_{0, \Omega_{t}}^{2} .
\end{aligned}
$$

Finally, we obtain inequalities for the fourth derivatives.

LEMma 3.9. Let $v, \varrho, \vartheta_{0}$ be a sufficiently smooth solution of (3.3). Then

$$
\begin{aligned}
\frac{1}{2} \frac{d}{d t} \int_{\Omega_{t}} & \left(\varrho v_{x x x}^{2}+\frac{p_{\sigma \varrho}}{\varrho} \varrho_{\sigma x x x}^{2}+\frac{\varrho c_{v}}{\theta} \vartheta_{0 x x x}^{2}\right) d x \\
& +c_{0}\left(\left\|v_{x x x}\right\|_{1, \Omega_{t}}^{2}+\left\|\varrho_{\sigma x x x}\right\|_{0, \Omega_{t}}^{2}+\left\|\vartheta_{0 x x x x}\right\|_{0, \Omega_{t}}^{2}\right) \\
\leq & \varepsilon\left(\left\|v_{x x x t}\right\|_{0, \Omega_{t}}^{2}+\left\|\vartheta_{0 x x x t}\right\|_{0, \Omega_{t}}^{2}\right) \\
& +C_{1}\left(|v|_{3,2, \Omega_{t}}^{2}+\left\|\varrho_{\sigma x}\right\|_{1, \Omega_{t}}^{2}+\left\|\bar{\varrho}_{\Omega_{t}}\right\|_{0, \Omega_{t}}^{2}+\left\|\vartheta_{0 x}\right\|_{2, \Omega_{t}}^{2}\right. \\
& \left.+\left\|\vartheta_{0 t}\right\|_{2, \Omega_{t}}^{2}+\|\vartheta\|_{0, \Omega_{t}}^{2}+\|f\|_{2, \Omega_{t}}^{2}+\|r\|_{2, \Omega_{t}}^{2}+\left\|\theta_{1}\right\|_{4, \Omega_{t}}^{2}\right) \\
& +C_{2}\left(X_{7}+\int_{0}^{t}\|v\|_{4, \Omega_{t^{\prime}}}^{2} d t^{\prime}\right)\left(1+X_{7}^{2}\right) Y_{7},
\end{aligned}
$$

where

$$
\begin{aligned}
X_{7} & =|v|_{3,2, \Omega_{t}}^{2}+\left|\varrho_{\sigma}\right|_{3,2, \Omega_{t}}^{2}+\left|\vartheta_{0}\right|_{3,2, \Omega_{t}}^{2}+\left\|\bar{\varrho}_{\Omega_{t}}\right\|_{0, \Omega_{t}}^{2}, \\
Y_{7} & =|v|_{4,3, \Omega_{t}}^{2}+\left|\varrho_{\sigma}\right|_{3,2, \Omega_{t}}^{2}+\left\|\vartheta_{0 x}\right\|_{3, \Omega_{t}}^{2}+\left\|\vartheta_{0 t}\right\|_{3, \Omega_{t}}^{2}+\|\vartheta\|_{0, \Omega_{t}}^{2}+\left\|\bar{\varrho}_{\Omega_{t}}\right\|_{0, \Omega_{t}}^{2} .
\end{aligned}
$$

Pr o of. We use the partition of unity. Differentiating $(3.46)_{1}$ and $(3.46)_{3}$ (divided by $\Gamma$ ) three times with respect to $\xi$, multiplying by $\widetilde{u}_{\xi \xi \xi} A$ and 
$\widetilde{\gamma}_{\xi \xi \xi} A$, respectively and next integrating over $\widetilde{\Omega}$ we get the estimate

$$
\begin{aligned}
\frac{1}{2} \frac{d}{d t} \int_{\tilde{\Omega}} & \left(\eta \widetilde{u}_{\xi \xi \xi}^{2}+\frac{p_{\sigma \eta}}{\eta} \widetilde{\bar{\eta}}_{\Omega_{t} \xi \xi \xi}^{2}+\frac{\eta c_{v}}{\Gamma} \widetilde{\gamma}_{\xi \xi \xi}^{2}\right) A d \xi \\
& +\frac{1}{2} \mu\left\|\widetilde{u}_{\xi \xi \xi}\right\|_{1, \tilde{\Omega}}^{2}+\left\|\widetilde{\bar{\eta}}_{\Omega_{t} \xi \xi \xi}\right\|_{0, \tilde{\Omega}}^{2}+\frac{\kappa}{\theta^{*}}\left\|\widetilde{\gamma}_{\xi \xi \xi \xi}\right\|_{0, \tilde{\Omega}}^{2} \\
\leq & \varepsilon\left(\left\|\widetilde{u}_{\xi \xi \xi \xi}\right\|_{0, \tilde{\Omega}}^{2}+\left\|\widetilde{\gamma}_{\xi \xi \xi \xi}\right\|_{0, \tilde{\Omega}}^{2}+\left\|\widetilde{\eta}_{\sigma \xi \xi \xi}\right\|_{0, \tilde{\Omega}}^{2}\right) \\
& +C_{1}\left(|u|_{3,2, \tilde{\Omega}}^{2}+\left\|\gamma_{0 \xi}\right\|_{2, \tilde{\Omega}}^{2}+\|\gamma\|_{0, \tilde{\Omega}}^{2}+\left\|\eta_{\sigma \xi}\right\|_{1, \tilde{\Omega}}^{2}\right. \\
& \left.+\left\|\bar{\eta}_{\Omega_{t}}\right\|_{0, \tilde{\Omega}}^{2}+\left\|\vartheta_{0 t}\right\|_{0, \Omega_{t}}^{2}+\|v\|_{1, \Omega_{t}}^{2}+\|\widetilde{g}\|_{2, \tilde{\Omega}}^{2}+\|\widetilde{k}\|_{2, \tilde{\Omega}}^{2}\right) \\
& +C_{2}\left[\left(X_{7}(\widetilde{\Omega})+\int_{0}^{t}\|u\|_{3, \tilde{\Omega}}^{2} d t^{\prime}\right)\left(1+X_{7}^{2}(\widetilde{\Omega})\right) Y_{7}(\widetilde{\Omega})\right. \\
& \left.+\|\gamma\|_{4, \tilde{\Omega}}^{2}\left(\left\|\vartheta_{0 t}\right\|_{0, \Omega_{t}}^{2}+\|v\|_{1, \Omega_{t}}^{2}\right)\right],
\end{aligned}
$$

where we have used equation (3.52), Lemma 5.1 of [21] in the case $G=\widetilde{\Omega}$, $v=\widetilde{u}_{\xi \xi \xi}$ and the estimate for the solution $u, \bar{\eta}_{\Omega_{t}}$ of the Stokes problem (3.57), i.e. the estimate of $\|\widetilde{u}\|_{4, \tilde{\Omega}}$ and $\left\|\widetilde{\bar{\eta}}_{\Omega_{t}}\right\|_{3, \tilde{\Omega}}$ respectively and

$$
\begin{aligned}
X_{7}(\widetilde{\Omega}) & =|u|_{3,2, \tilde{\Omega}}^{2}+\left|\bar{\eta}_{\Omega_{t}}\right|_{3,2, \tilde{\Omega}}^{2}+|\gamma|_{3,2, \tilde{\Omega}}^{2}+\left|\eta_{\sigma}\right|_{3,2, \tilde{\Omega}}^{2}+\left|\gamma_{0}\right|_{3,2, \tilde{\Omega}}^{2}, \\
Y_{7}(\widetilde{\Omega}) & =|u|_{4,3, \tilde{\Omega}}^{2}+\left|\bar{\eta}_{\Omega_{t}}\right|_{3,2, \tilde{\Omega}}^{2}+|\gamma|_{4,3, \tilde{\Omega}}^{2}+\left|\eta_{\sigma t}\right|_{2,1, \tilde{\Omega}}^{2}+\|\left.\gamma_{0 t}\right|_{3, \tilde{\Omega}} ^{2}
\end{aligned}
$$

For boundary subdomains we obtain

$$
\begin{aligned}
\frac{1}{2} \frac{d}{d t} \int_{\hat{\Omega}} & \left(\widehat{\eta} \widetilde{u}_{\tau \tau \tau}^{2}+\frac{p_{\sigma \hat{\eta}}}{\widehat{\eta}} \widetilde{\bar{\eta}}_{\Omega_{t} \tau \tau \tau}^{2}+\frac{\widehat{\eta} c_{v}}{\widehat{\Gamma}} \widetilde{\gamma}_{\tau \tau \tau}^{2}\right) J d z \\
& +\frac{1}{2} \mu\left\|\widetilde{u}_{\tau \tau \tau}\right\|_{1, \hat{\Omega}}^{2}+\frac{\kappa}{\theta^{*}}\left\|\widetilde{\gamma}_{\tau \tau \tau z}\right\|_{0, \hat{\Omega}}^{2} \\
\leq & \varepsilon\left(\left\|\widehat{u}_{z z z z}\right\|_{0, \hat{\Omega}}^{2}+\left\|\widehat{\gamma}_{0 z z z z}\right\|_{0, \hat{\Omega}}^{2}+\left\|\widehat{\eta}_{\sigma z z z}\right\|_{0, \hat{\Omega}}^{2}\right) \\
& +C_{1}\left(|\widehat{u}|_{3,2, \hat{\Omega}}^{2}+\left\|\widehat{\gamma}_{0 z}\right\|_{2, \hat{\Omega}}^{2}+\|\widehat{\gamma}\|_{0, \hat{\Omega}}^{2}+\left\|\widehat{\eta}_{\sigma z}\right\|_{1, \hat{\Omega}}^{2}+\left\|\bar{\eta}_{\Omega_{t}}\right\|_{0, \hat{\Omega}}^{2}\right. \\
& \left.+\left\|\vartheta_{0 t}\right\|_{0, \Omega_{t}}^{2}+\|v\|_{1, \Omega_{t}}^{2}+\|\widetilde{g}\|_{2, \hat{\Omega}}^{2}+\|\widetilde{k}\|_{2, \hat{\Omega}}^{2}+\left\|\widetilde{\Gamma}_{1}\right\|_{4, \hat{\Omega}}^{2}\right) \\
& +C_{2}\left[\left(X_{7}(\widehat{\Omega})+\int_{0}^{t}\|\widehat{u}\|_{3, \hat{\Omega}}^{2} d t^{\prime}\right)\left(1+X_{7}^{2}(\widehat{\Omega})\right) Y_{7}(\widehat{\Omega})\right. \\
& \left.+\|\widehat{\gamma}\|_{4, \hat{\Omega}}^{2}\left(\left\|\vartheta_{0 t}\right\|_{0, \Omega_{t}}^{2}+\|v\|_{1, \Omega_{t}}^{2}\right)\right],
\end{aligned}
$$

where we have used the boundary conditions $(3.47)_{4}$ and $(3.47)_{5}$, and where $X_{7}(\widehat{\Omega})$ and $Y_{7}(\widehat{\Omega})$ are defined by (3.127) with $\widetilde{\Omega}, u, \bar{\eta}_{\Omega_{t}}, \gamma$ replaced by $\widehat{\Omega}$, $\widehat{u}, \widehat{\bar{\eta}}_{\Omega_{t}}, \widehat{\gamma}$, respectively. 
In the same way as (3.92) and (3.93) we obtain the following estimates:

$$
\begin{aligned}
& \frac{1}{2} \frac{d}{d t} \int_{\hat{\Omega}} \frac{p_{\sigma \hat{\eta}}}{\widehat{\eta}} \overline{\bar{\eta}}_{\Omega_{t} n \tau \tau}^{2} J d z+c_{0}\left\|\widetilde{\bar{\eta}}_{\Omega_{t} n \tau \tau}\right\|_{0, \hat{\Omega}}^{2} \\
& \leq(\varepsilon+c d)\left(\left\|\widetilde{\bar{\eta}}_{\Omega_{t} n \tau \tau}\right\|_{0, \hat{\Omega}}^{2}+\left\|\widetilde{u}_{z z \tau \tau}\right\|_{0, \hat{\Omega}}^{2}\right) \\
&+C_{1}\left(\left\|\widetilde{u}_{\tau \tau \tau}\right\|_{1, \hat{\Omega}}^{2}+|\widehat{u}|_{3,2, \hat{\Omega}}^{2}+\left\|\widehat{\eta}_{\sigma z}\right\|_{1, \hat{\Omega}}^{2}+\left\|\widehat{\bar{\eta}}_{\Omega_{t}}\right\|_{0, \hat{\Omega}}^{2}\right. \\
&\left.+\left\|\widehat{\gamma}_{0 z}\right\|_{2, \hat{\Omega}}^{2}+\|\widehat{\gamma}\|_{0, \hat{\Omega}}^{2}+\left\|\vartheta_{0 t}\right\|_{0, \Omega_{t}}^{2}+\|v\|_{1, \Omega_{t}}^{2}+\|\widetilde{g}\|_{2, \hat{\Omega}}^{2}\right) \\
&+C_{2}\left[\left(X_{7}(\widehat{\Omega})+\int_{0}^{t}\|\widehat{u}\|_{3, \hat{\Omega}}^{2} d t^{\prime}\right)\left(1+X_{7}^{2}(\widehat{\Omega})\right) Y_{7}(\widehat{\Omega})\right. \\
&+\left(\left\|\widehat{\eta}_{\sigma}\right\|_{2, \hat{\Omega}}^{2}+\left\|\widehat{\gamma}_{0}\right\|_{2, \hat{\Omega}}^{2}+\left\|\widehat{\eta}_{\sigma}\right\|_{2, \hat{\Omega}}^{4}\right. \\
&\left.\left.+\left\|\widehat{\eta}_{\sigma}\right\|_{2, \hat{\Omega}}^{2}\left\|\widehat{\gamma}_{0}\right\|_{2, \hat{\Omega}}^{2}+\left\|\widehat{\gamma}_{0}\right\|_{2, \hat{\Omega}}^{4}\right)\left(\left\|\vartheta_{0 t}\right\|_{0, \Omega_{t}}^{2}+\|v\|_{1, \Omega_{t}}^{2}\right)\right]
\end{aligned}
$$

and

$(3.130)$

$$
\begin{aligned}
\frac{1}{2} \frac{d}{d t} \int_{\hat{\Omega}} \widehat{\eta}\left|\widetilde{u}_{3 n \tau \tau}\right|^{2} J d z+c_{0}\left\|\widetilde{u}_{3 n n \tau \tau}\right\|_{0, \hat{\Omega}}^{2} \\
\leq(\varepsilon+c d)\left(\left\|\widetilde{u}_{z z \tau \tau}\right\|_{0, \hat{\Omega}}^{2}+\left\|\widetilde{\bar{\eta}}_{\Omega_{t} z \tau \tau}\right\|_{0, \hat{\Omega}}^{2}\right)+\varepsilon\left\|\widetilde{u}_{n \tau \tau t}\right\|_{0, \hat{\Omega}}^{2} \\
\quad+C_{1}\left(\left\|\widetilde{u}_{z \tau \tau \tau}\right\|_{0, \hat{\Omega}}^{2}+\left\|\widetilde{\bar{\eta}}_{\Omega_{t} n \tau \tau}\right\|_{0, \hat{\Omega}}^{2}+|\widehat{u}|_{3,2, \hat{\Omega}}^{2}+\left\|\widehat{\eta}_{\sigma z}\right\|_{1, \hat{\Omega}}^{2}\right. \\
\left.\quad+\left\|\widehat{\bar{\eta}}_{\Omega_{t}}\right\|_{0, \hat{\Omega}}^{2}+\left\|\widehat{\gamma}_{0 z}\right\|_{2, \hat{\Omega}}^{2}+\|\widehat{\gamma}\|_{0, \hat{\Omega}}^{2}+\|\widetilde{g}\|_{2, \hat{\Omega}}^{2}\right) \\
\quad+C_{2}\left(X_{7}(\widehat{\Omega})+\int_{0}^{t}\|\widehat{u}\|_{3, \hat{\Omega}}^{2} d t^{\prime}\right)\left(1+X_{7}^{2}(\widehat{\Omega})\right) Y_{7}(\widehat{\Omega})
\end{aligned}
$$

Next, differentiating (3.68) three times with respect to $\tau$, multiplying by $\widetilde{u}_{\tau \tau \tau}^{\prime} J$, integrating over $\widehat{\Omega}$ and using the boundary condition (3.69) we get

$$
\begin{aligned}
& \left\|\widetilde{u}_{z \tau \tau \tau}^{\prime}\right\|_{0, \hat{\Omega}}^{2}+\left\|\widetilde{\bar{\eta}}_{\Omega_{t} \tau \tau \tau}\right\|_{0, \hat{\Omega}}^{2} \\
& \leq(\varepsilon+c d)\left(\left\|\widehat{u}_{z z z z}\right\|_{0, \hat{\Omega}}^{2}+\left\|\widehat{\bar{\eta}}_{\Omega_{t} z z z}\right\|_{0, \hat{\Omega}}^{2}\right)+C_{1}\left(\left\|\operatorname{div} \widetilde{u}_{\tau \tau}\right\|_{1, \hat{\Omega}}^{2}+|\widehat{u}|_{3,2, \hat{\Omega}}^{2}\right. \\
& \left.\quad+\left\|\widehat{\eta}_{\sigma z}\right\|_{1, \hat{\Omega}}^{2}+\left\|\widehat{\bar{\eta}}_{\Omega_{t}}\right\|_{0, \hat{\Omega}}^{2}+\left\|\widehat{\gamma}_{0 z}\right\|_{2, \hat{\Omega}}^{2}+\|\widehat{\gamma}\|_{0, \hat{\Omega}}^{2}+\|\widetilde{g}\|_{2, \hat{\Omega}}^{2}\right) \\
& \quad+C_{2}\left(X_{7}(\widehat{\Omega})+\int_{0}^{t}\|\widehat{u}\|_{3, \hat{\Omega}}^{2} d t^{\prime}\right)\left(1+X_{7}^{2}(\widehat{\Omega})\right) Y_{7}(\widehat{\Omega}) .
\end{aligned}
$$

Moreover, from (3.68) we find 
$(3.132)$

$$
\begin{aligned}
& \left\|\widetilde{u}_{n n \tau \tau}^{\prime}\right\|_{0, \hat{\Omega}}^{2} \leq(\varepsilon+c d)\left(\left\|\widetilde{u}_{z z z z}\right\|_{0, \hat{\Omega}}^{2}+\left\|\widehat{\bar{\eta}}_{\Omega_{t} z z z}\right\|_{0, \hat{\Omega}}^{2}\right) \\
& \quad+C_{1}\left(\left\|\widetilde{u}_{\tau \tau \tau \tau}^{\prime}\right\|_{0, \hat{\Omega}}^{2}+\left\|(\operatorname{div} \widetilde{u})_{, \tau \tau \tau}\right\|_{0, \hat{\Omega}}^{2}+\left\|\overline{\bar{\eta}}_{\Omega_{t} \tau \tau \tau}\right\|_{0, \hat{\Omega}}^{2}+|\widehat{u}|_{3,2, \hat{\Omega}}^{2}\right. \\
& \left.\quad+\left\|\widehat{\eta}_{\sigma z}\right\|_{1, \hat{\Omega}}^{2}+\left\|\overline{\bar{\eta}}_{\Omega_{t}}\right\|_{0, \hat{\Omega}}^{2}+\left\|\widehat{\gamma}_{0 z}\right\|_{2, \hat{\Omega}}^{2}+\|\widehat{\gamma}\|_{0, \hat{\Omega}}^{2}+\|\widetilde{g}\|_{2, \hat{\Omega}}^{2}\right) \\
& \quad+C_{2}\left(X_{7}(\widehat{\Omega})+\int_{0}^{t}\|\widehat{u}\|_{3, \hat{\Omega}}^{2} d t^{\prime}\right)\left(1+X_{7}^{2}(\widehat{\Omega})\right) Y_{7}(\widehat{\Omega}) .
\end{aligned}
$$

Next, dividing (3.84) by $\widehat{\Gamma}$, differentiating twice with respect to $\tau$, multiplying the result by $\widetilde{\gamma}_{n n \tau \tau} J$ and integrating over $\widehat{\Omega}$ gives

$$
\begin{aligned}
& \frac{1}{2} \frac{d}{d t} \int_{\hat{\Omega}} \frac{\widehat{\eta} c_{v}}{\widehat{\Gamma}} \widetilde{\gamma}_{n \tau \tau}^{2} J d z+\frac{\kappa}{\theta^{*}} \int_{\hat{\Omega}} \widetilde{\gamma}_{n n \tau \tau}^{2} J d z \\
& \leq(\varepsilon+c d)\left(\left\|\widetilde{\gamma}_{z z z z}\right\|_{0, \hat{\Omega}}^{2}+\left\|\widetilde{\bar{\eta}}_{\Omega_{t} z z z}\right\|_{0, \hat{\Omega}}^{2}\right)+\varepsilon\left\|\widetilde{\gamma}_{n \tau \tau t}\right\|_{0, \hat{\Omega}}^{2} \\
&+C_{1}\left(\|\widetilde{u}\|_{3, \hat{\Omega}}^{2}+\left\|\widetilde{\gamma}_{z \tau \tau \tau}\right\|_{0, \hat{\Omega}}^{2}+\left\|\widehat{\gamma}_{0 z}\right\|_{2, \hat{\Omega}}^{2}\right. \\
&\left.+\left\|\widehat{\gamma}_{0, \hat{\Omega}}^{2}+\right\| \vartheta_{0 t}\left\|_{0, \Omega_{t}}^{2}+\right\| v\left\|_{1, \Omega_{t}}^{2}+\right\| \widetilde{g} \|_{2, \hat{\Omega}}^{2}\right) \\
&+C_{2}\left[\left(X_{7}(\widehat{\Omega})+\int_{0}^{t}\|\widehat{u}\|_{3, \hat{\Omega}}^{2} d t^{\prime}\right)\left(1+X_{7}^{2}(\widehat{\Omega})\right) Y_{7}(\widehat{\Omega})\right. \\
&+\left(\left\|\widehat{\eta}_{\sigma}\right\|_{2, \hat{\Omega}}^{2}+\left\|\widehat{\gamma}_{0}\right\|_{2, \hat{\Omega}}^{2}+\left\|\widehat{\eta}_{\sigma}\right\|_{2, \hat{\Omega}}^{4}\right. \\
&\left.\left.+\left\|\widehat{\eta}_{\sigma}\right\|_{2, \hat{\Omega}}^{2}\left\|\widehat{\gamma}_{0}\right\|_{2, \hat{\Omega}}^{2}+\left\|\widehat{\gamma}_{0}\right\|_{2, \hat{\Omega}}^{4}\right)\left(\left\|\vartheta_{0 t}\right\|_{1, \Omega_{t}}^{2}+\|v\|_{2, \Omega_{t}}^{2}\right)\right] .
\end{aligned}
$$

Differentiating the third component of (3.64) with respect to $n$ and $\tau$, multiplying by $\widetilde{\bar{\eta}}_{\Omega_{t} n n \tau} J$ and integrating over $\widehat{\Omega}$ yields

$$
\begin{aligned}
& \frac{1}{2} \frac{d}{d t} \int_{\hat{\Omega}} \frac{\varrho_{\sigma \hat{\eta}}}{\widehat{\eta}} \overline{\bar{\eta}}_{\Omega_{t} n n \tau} J d z+c_{0}\left\|\widetilde{\bar{\eta}}_{\Omega_{t} n n \tau}\right\|_{0, \hat{\Omega}}^{2} \\
& \leq(\varepsilon+c d)\left(\left\|\widetilde{u}_{z z z \tau}\right\|_{0, \hat{\Omega}}^{2}+\left\|\widetilde{\bar{\eta}}_{\Omega_{t} z z}\right\|_{0, \hat{\Omega}}^{2}\right) \\
& \quad+C_{1}\left(\left\|\widetilde{u}_{z z \tau \tau}\right\|_{0, \hat{\Omega}}^{2}+|\widehat{u}|_{3,2, \hat{\Omega}}^{2}+\left\|\widehat{\eta}_{\sigma z}\right\|_{1, \hat{\Omega}}^{2}+\left\|\widehat{\eta}_{\sigma t}\right\|_{1, \hat{\Omega}}^{2}+\left\|\bar{\eta}_{\Omega_{t}}\right\|_{0, \hat{\Omega}}^{2}\right. \\
& \left.\quad+\left\|\widehat{\gamma}_{0 z}\right\|_{2, \hat{\Omega}}^{2}+\|\widehat{\gamma}\|_{0, \hat{\Omega}}^{2}+\left\|\vartheta_{0 t}\right\|_{0, \Omega_{t}}^{2}+\|v\|_{1, \Omega_{t}}^{2}+\|\widetilde{g}\|_{2, \hat{\Omega}}^{2}\right) \\
& \quad+C_{2}\left[\left(X_{7}(\widehat{\Omega})+\int_{0}^{t}\|\widehat{u}\|_{3, \hat{\Omega}}^{2} d t^{\prime}\right)\left(1+X_{7}^{2}(\widehat{\Omega})\right) Y_{7}(\widehat{\Omega})\right. \\
& \quad+\left(\left\|\widehat{\eta}_{\sigma}\right\|_{2, \hat{\Omega}}^{2}+\left\|\widehat{\gamma}_{0}\right\|_{2, \hat{\Omega}}^{2}+\left\|\widehat{\eta}_{\sigma}\right\|_{2, \hat{\Omega}}^{4}\right. \\
& \left.\left.\quad+\left\|\widehat{\eta}_{\sigma}\right\|_{2, \hat{\Omega}}^{2}\left\|\widehat{\gamma}_{0}\right\|_{2, \hat{\Omega}}^{2}+\left\|\widehat{\gamma}_{0}\right\|_{2, \hat{\Omega}}^{4}\right)\left(\left\|\vartheta_{0 t}\right\|_{1, \Omega_{t}}^{2}+\|v\|_{2, \Omega_{t}}^{2}\right)\right] .
\end{aligned}
$$


Differentiating the third component of (3.76) and next (3.68) with respect to $n$ and $\tau$ we have respectively

(3.135) $\quad\left\|(\operatorname{div} \widetilde{u})_{, n n \tau}\right\|_{0, \hat{\Omega}}^{2}$

$$
\begin{aligned}
\leq & (\varepsilon+c d)\left\|\widetilde{u}_{z z z z}\right\|_{0, \hat{\Omega}}^{2}+C_{1}\left(\left\|\widetilde{u}_{z z \tau \tau}\right\|_{0, \hat{\Omega}}^{2}+\left\|\widetilde{\bar{\eta}}_{\Omega_{t} n n \tau}\right\|_{0, \hat{\Omega}}^{2}\right. \\
& \left.+|\widehat{u}|_{3,2, \hat{\Omega}}^{2}+\left\|\widehat{\eta}_{\sigma z}\right\|_{0, \hat{\Omega}}^{2}+\left\|\widehat{\bar{\eta}}_{\Omega_{t}}\right\|_{0, \hat{\Omega}}^{2}+\left\|\widehat{\gamma}_{0 z}\right\|_{2, \hat{\Omega}}^{2}+\|\widehat{\gamma}\|_{0, \hat{\Omega}}^{2}\right) \\
& +C_{2}\left(X_{7}(\widehat{\Omega})+\int_{0}^{t}\|\widehat{u}\|_{3, \hat{\Omega}}^{2} d t^{\prime}\right)\left(1+X_{7}^{2}(\widehat{\Omega})\right) Y_{7}(\widehat{\Omega})
\end{aligned}
$$

and

(3.136)

$$
\begin{aligned}
& \left\|\widetilde{u}_{n n n \tau}\right\|_{0, \hat{\Omega}}^{2} \\
& \leq \quad \\
& \quad+c d)\left(\left\|\widetilde{u}_{z z z z}\right\|_{0, \hat{\Omega}}^{2}+\left\|\widetilde{\bar{\eta}}_{\Omega_{t} z z z}\right\|_{0, \hat{\Omega}}^{2}\right) \\
& \quad+C_{1}\left(\left\|\widetilde{u}_{z z \tau \tau}\right\|_{0, \hat{\Omega}}^{2}+\left\|(\operatorname{div} \widetilde{u})_{, z n \tau}\right\|_{0, \hat{\Omega}}^{2}+\left\|\widehat{\bar{\eta}}_{\Omega_{t} z n \tau}\right\|_{0, \hat{\Omega}}^{2}+|\widehat{u}|_{3,2, \hat{\Omega}}^{2}\right. \\
& \left.\quad+\left\|\widehat{\eta}_{\sigma z}\right\|_{1, \hat{\Omega}}^{2}+\left\|\overline{\bar{\eta}}_{\Omega_{t}}\right\|_{0, \hat{\Omega}}^{2}+\left\|\widehat{\gamma}_{0 z}\right\|_{2, \hat{\Omega}}^{2}+\|\widehat{\gamma}\|_{0, \hat{\Omega}}^{2}+\|\widetilde{g}\|_{2, \hat{\Omega}}^{2}\right) \\
& \quad+C_{2}\left(X_{7}(\widehat{\Omega})+\int_{0}^{t}\|\widehat{u}\|_{3, \hat{\Omega}}^{2} d t^{\prime}\right)\left(1+X_{7}^{2}(\widehat{\Omega})\right) Y_{7}(\widehat{\Omega}) .
\end{aligned}
$$

Now, we rewrite equation (3.84) as

$$
\begin{aligned}
-\kappa \Delta \widetilde{\gamma}= & -\widehat{\eta} c_{v} \widetilde{\gamma}_{t}+\kappa \widehat{\nabla}^{2} \widetilde{\gamma}-\kappa \Delta \widetilde{\gamma} \\
& -\widehat{\Gamma} p_{\hat{\Gamma}} \widehat{\nabla} \cdot \widetilde{u}+\widehat{\eta} \widetilde{k}+k_{6} .
\end{aligned}
$$

Differentiating (3.137) with respect to $n$ and $\tau$ and multiplying the result by $\widetilde{\gamma}_{n n n \tau} J$ we have

$$
\begin{aligned}
\text { (3.138) }\left\|\widetilde{\gamma}_{n n n \tau}\right\|_{0, \hat{\Omega}}^{2} \leq & (\varepsilon+c d)\left\|\widetilde{\gamma}_{z z z z}\right\|_{0, \hat{\Omega}}^{2} \\
& +C_{1}\left(\left\|\widetilde{\gamma}_{z z \tau \tau}\right\|_{0, \hat{\Omega}}^{2}+\|\widehat{u}\|_{3, \hat{\Omega}}^{2}+\left\|\widehat{\eta}_{\sigma z}\right\|_{1, \hat{\Omega}}^{2}\right. \\
& +\left\|\widehat{\bar{\eta}}_{\Omega_{t}}\right\|_{0, \hat{\Omega}}^{2}+\left\|\widehat{\gamma}_{0 z}\right\|_{2, \hat{\Omega}}^{2}+\left\|\widehat{\gamma}_{0 t}\right\|_{2, \hat{\Omega}}^{2}+\|\widehat{\gamma}\|_{0, \hat{\Omega}}^{2} \\
& \left.+\left\|\vartheta_{0 t}\right\|_{0, \Omega_{t}}^{2}+\|v\|_{1, \Omega_{t}}^{2}+\|\widetilde{k}\|_{2, \hat{\Omega}}^{2}\right) \\
& +C_{2}\left[\left(X_{7}(\widehat{\Omega})+\int_{0}^{t}\|\widehat{u}\|_{3, \hat{\Omega}}^{2} d t^{\prime}\right)\left(1+X_{7}^{2}(\widehat{\Omega})\right) Y_{7}(\widehat{\Omega})\right. \\
& +\left(\left\|\widehat{\eta}_{\sigma}\right\|_{2, \hat{\Omega}}^{2}+\left\|\widehat{\gamma}_{0}\right\|_{2, \hat{\Omega}}^{2}+\left\|\widehat{\eta}_{\sigma}\right\|_{2, \hat{\Omega}}^{4}\right. \\
& \left.\left.+\left\|\widehat{\eta}_{\sigma}\right\|_{2, \hat{\Omega}}^{2}\left\|\widehat{\gamma}_{0}\right\|_{2, \hat{\Omega}}^{2}+\left\|\widehat{\gamma}_{0}\right\|_{2, \hat{\Omega}}^{4}\right)\left(\left\|\vartheta_{0 t}\right\|_{1, \Omega_{t}}^{2}+\|v\|_{2, \Omega_{t}}^{2}\right)\right] .
\end{aligned}
$$

Next, differentiating the third components of problems (3.64), (3.76), 
(3.68) and problem (3.137) twice with respect to $n$ we get the estimates for

$$
\begin{gathered}
\frac{1}{2} \frac{d}{d t} \int_{\hat{\Omega}} \frac{p_{\sigma \hat{\eta}}}{\widehat{\eta}} \widetilde{\bar{\eta}}_{\Omega_{t} n n n} J d z+\left\|\widetilde{\bar{\eta}}_{\Omega_{t} n n n}\right\|_{0, \hat{\Omega}}^{2}, \quad\left\|(\operatorname{div} \widetilde{u})_{, n n n}\right\|_{0, \hat{\Omega}}^{2}, \\
\left\|\widetilde{u}_{n n n n}\right\|_{0, \hat{\Omega}}^{2} \text { and }\left\|\widetilde{\gamma}_{n n n n}\right\|_{0, \hat{\Omega}}^{2},
\end{gathered}
$$

which are analogous to (3.134)-(3.136) and (3.138), respectively.

Finally, we have

$$
\frac{1}{2} \frac{d}{d t} \int_{\hat{\Omega}} \widehat{\eta} \widetilde{u}_{z z z}^{2} J d z \leq \varepsilon\left\|\widetilde{u}_{z z z t}\right\|_{0, \hat{\Omega}}^{2}+C_{1}\|\widetilde{u}\|_{3, \hat{\Omega}}^{2}
$$

and

(3.140) $\quad \frac{1}{2} \frac{d}{d t} \int_{\hat{\Omega}} \frac{\widehat{\eta} c_{v}}{\widehat{\Gamma}} \widetilde{\gamma}_{z z z}^{2} J d z \leq \varepsilon\left\|\widetilde{\gamma}_{z z z t}\right\|_{0, \hat{\Omega}}^{2}+C_{1}\left(\left\|\widehat{\gamma}_{0 z}\right\|_{2, \hat{\Omega}}^{2}+\|\widehat{\gamma}\|_{0, \hat{\Omega}}^{2}\right)$

$$
+C_{2}\left[\left(\left|\widehat{\eta}_{\sigma}\right|_{2,1, \hat{\Omega}}^{2}+\left|\widehat{\gamma}_{0}\right|_{2,1, \hat{\Omega}}^{2}+\|\widehat{u}\|_{2, \hat{\Omega}}^{2}\right)\|\widehat{\gamma}\|_{4, \hat{\Omega}}^{2}\right] .
$$

The above considerations yield

$$
\begin{aligned}
& \frac{1}{2} \frac{d}{d t} \int_{\hat{\Omega}}\left(\widehat{\eta} \widetilde{u}_{z z z}^{2}+\frac{p_{\sigma \hat{\eta}}}{\widehat{\eta}} \widetilde{\eta}_{\sigma z z z}^{2}+\frac{\widehat{\eta} c_{v}}{\widehat{\Gamma}} \widetilde{\gamma}_{z z z}^{2}\right) J d z \\
& \quad+c_{0}\left(\left\|\widetilde{u}_{z z z}\right\|_{1, \hat{\Omega}}^{2}+\left\|\widetilde{\bar{\eta}}_{\Omega_{t} z z z}\right\|_{0, \hat{\Omega}}^{2}+\left\|\widetilde{\gamma}_{z z z z}\right\|_{0, \hat{\Omega}}^{2}\right) \\
& \leq(\varepsilon+c d)\left(\left\|\widetilde{u}_{z z z z}\right\|_{0, \hat{\Omega}}^{2}+\left\|\widetilde{\bar{\eta}}_{\Omega_{t} z z z}\right\|_{0, \hat{\Omega}}^{2}+\left\|\widetilde{\gamma}_{z z z z}\right\|_{0, \hat{\Omega}}^{2}\right) \\
& \quad+\varepsilon\left\|\widetilde{u}_{z z z t}\right\|_{0, \hat{\Omega}}^{2}+C_{1}\left(|\widehat{u}|_{3,2, \hat{\Omega}}^{2}+\left\|\widehat{\gamma}_{0 z}\right\|_{2, \hat{\Omega}}^{2}+\|\widehat{\gamma}\|_{0, \hat{\Omega}}^{2}+\left\|\widehat{\eta}_{\sigma z}\right\|_{1, \hat{\Omega}}^{2}\right. \\
& \left.\quad+\left\|\widehat{\bar{\eta}}_{\Omega_{t}}\right\|_{0, \hat{\Omega}}^{2}+\left\|\vartheta_{0 t}\right\|_{0, \Omega_{t}}^{2}+\|v\|_{1, \Omega_{t}}^{2}+\|\widetilde{g}\|_{2, \hat{\Omega}}^{2}+\|\widetilde{k}\|_{2, \hat{\Omega}}^{2}+\left\|\widetilde{\Gamma}_{1}\right\|_{4, \hat{\Omega}}^{2}\right) \\
& \quad+C_{2}\left[\left(X_{7}(\widehat{\Omega})+\int_{0}^{t}\|\widehat{u}\|_{3, \hat{\Omega}}^{2} d t^{\prime}\right)\left(1+X_{7}^{2}(\widehat{\Omega})\right) Y_{7}(\widehat{\Omega})\right. \\
& \quad+\left(\left\|\widehat{\eta}_{\sigma}\right\|_{2, \hat{\Omega}}^{2}+\left\|\widehat{\gamma}_{0}\right\|_{2, \hat{\Omega}}^{2}+\left\|\widehat{\eta}_{\sigma}\right\|_{2, \hat{\Omega}}^{4}\right. \\
& \left.\left.\quad+\left\|\widehat{\eta}_{\sigma}\right\|_{2, \hat{\Omega}}^{2}\left\|\widehat{\gamma}_{0}\right\|_{2, \hat{\Omega}}^{2}+\left\|\widehat{\gamma}_{0}\right\|_{2, \hat{\Omega}}^{2}\right)\left(\left\|\vartheta_{0 t}\right\|_{1, \Omega_{t}}^{2}+\|v\|_{2, \Omega_{t}}^{2}\right)\right] .
\end{aligned}
$$

By estimates (3.126) and (3.141) we obtain the assertion of the lemma.

In order to estimate the first term on the right-hand side of (3.125) we need the following lemma.

Lemma 3.10. Let $v, \varrho, \vartheta_{0}$ be a sufficiently smooth solution of prob- 
lem (3.3). Then

$$
\begin{aligned}
(3.142) & \frac{1}{2} \frac{d}{d t} \int_{\Omega_{t}}\left(\varrho v_{x x t}^{2}+\frac{p_{\sigma \varrho}}{\varrho} \varrho_{\sigma x x t}^{2}+\frac{\varrho c_{v}}{\theta} \vartheta_{0 x x t}^{2}\right) d x \\
& +c_{0}\left(\left\|v_{x x t}\right\|_{1, \Omega_{t}}^{2}+\left\|\varrho_{\sigma x x t}\right\|_{0, \Omega_{t}}^{2}+\left\|\vartheta_{0 x x x t}\right\|_{0, \Omega_{t}}^{2}\right) \\
\leq & \varepsilon\left(\left\|v_{x x t t}\right\|_{0, \Omega_{t}}^{2}+\left\|v_{x x x x}\right\|_{0, \Omega_{t}}^{2}+\left\|\vartheta_{0 x x x x}\right\|_{0, \Omega_{t}}^{2}+\left\|\vartheta_{0 x x t t}\right\|_{0, \Omega_{t}}^{2}\right) \\
& +C_{1}\left(|v|_{3,1, \Omega_{t}}^{2}+\left\|\varrho_{\sigma x}\right\|_{1, \Omega_{t}}^{2}+\left\|\varrho_{\sigma t}\right\|_{0, \Omega_{t}}^{2}+\left\|\varrho_{\Omega_{t}}\right\|_{0, \Omega_{t}}^{2}+\left\|\vartheta_{0 x}\right\|_{2, \Omega_{t}}^{2}\right. \\
& \left.+\left\|\vartheta_{0 t}\right\|_{2, \Omega_{t}}^{2}+\|\vartheta\|_{0, \Omega_{t}}^{2}+|f|_{2,1, \Omega_{t}}^{2}+|r|_{2,1, \Omega_{t}}^{2}+\left\|\theta_{1 t}\right\|_{3, \Omega_{t}}^{2}+\left\|\theta_{1}\right\|_{3, \Omega_{t}}^{2}\right) \\
& +C_{2}\left(X_{8}+\int_{0}^{t}\|v\|_{4, \Omega_{t^{\prime}}}^{2} d t^{\prime}\right)\left(1+X_{8}^{2}\right) Y_{8},
\end{aligned}
$$

where

$$
\begin{aligned}
X_{8} & =|v|_{3,2, \Omega_{t}}^{2}+\left|\varrho_{\sigma}\right|_{3,1, \Omega_{t}}^{2}+\left|\vartheta_{0}\right|_{3,1, \Omega_{t}}^{2}+\left\|\bar{\varrho}_{\Omega_{t}}\right\|_{0, \Omega_{t}}^{2}, \\
Y_{8} & =|v|_{4,3, \Omega_{t}}^{2}+\left|\varrho_{\sigma}\right|_{3,1, \Omega_{t}}^{2}+\left\|\vartheta_{0 x}\right\|_{3, \Omega_{t}}^{2}+\left\|\vartheta_{0 t}\right\|_{3, \Omega_{t}}^{2}+\|\vartheta\|_{0, \Omega_{t}}^{2}+\left\|\bar{\varrho}_{\Omega_{t}}\right\|_{0, \Omega_{t}}^{2} .
\end{aligned}
$$

Proof. The proof is analogous to the proofs of Lemmas 3.4-3.6 and 3.9.

To estimate the first term on the right-hand side of (3.142) we need the following result.

LEMma 3.11. Let $v, \varrho, \vartheta_{0}$ be a sufficiently smooth solution of problem (3.3). Then

$$
\begin{aligned}
\frac{1}{2} \frac{d}{d t} \int_{\Omega_{t}} & \left(\varrho v_{x t t}^{2}+\frac{p_{\sigma \varrho}}{\varrho} \varrho_{\sigma x t t}^{2}+\frac{\varrho c_{v}}{\theta} \vartheta_{0 x t t}^{2}\right) d x \\
& +c_{0}\left(\left\|v_{t t x}\right\|_{1, \Omega_{t}}^{2}+\left\|\varrho_{\sigma t t}\right\|_{1, \Omega_{t}}^{2}+\left\|\vartheta_{0 t t x x}\right\|_{0, \Omega_{t}}^{2}\right) \\
\leq & \varepsilon\left(\left\|v_{x x x t}\right\|_{0, \Omega_{t}}^{2}+\left\|v_{x t t t}\right\|_{0, \Omega_{t}}^{2}+\left\|\vartheta_{0 x x x t}\right\|_{0, \Omega_{t}}^{2}+\left\|\vartheta_{0 x t t}\right\|_{0, \Omega_{t}}^{2}\right) \\
& +C_{1}\left(|v|_{3,0, \Omega_{t}}^{2}+\left\|\varrho_{\sigma x}\right\|_{1, \Omega_{t}}^{2}+\left\|\varrho_{\sigma t}\right\|_{1, \Omega_{t}}^{2}+\left\|\varrho_{\Omega_{t}}\right\|_{0, \Omega_{t}}^{2}\right. \\
& +\left\|\vartheta_{0 x}\right\|_{2, \Omega_{t}}^{2}+\left|\vartheta_{0 t}\right|_{2,0, \Omega_{t}}^{2}+\|\vartheta\|_{0, \Omega_{t}}^{2}+|f|_{2,0, \Omega_{t}}^{2} \\
& \left.+|r|_{2,0, \Omega_{t}}^{2}+\left\|\theta_{1 t t}\right\|_{2, \Omega_{t}}^{2}+\left\|\theta_{1 t}\right\|_{2, \Omega_{t}}^{2}+\left\|\theta_{1}\right\|_{2, \Omega_{t}}^{2}\right) \\
& +C_{2}\left(X_{9}+\int_{0}^{t}\|v\|_{4, \Omega_{t^{\prime}}}^{2} d t^{\prime}\right)\left(1+X_{9}^{2}\right) Y_{9},
\end{aligned}
$$

where

$$
\begin{aligned}
X_{9} & =|v|_{3,0, \Omega_{t}}^{2}+\left|\varrho_{\sigma}\right|_{3,0, \Omega_{t}}^{2}+\left|\vartheta_{0}\right|_{3,0, \Omega_{t}}^{2}+\left\|\bar{\varrho}_{\Omega_{t}}\right\|_{0, \Omega_{t}}^{2}, \\
Y_{9} & =|v|_{4,1, \Omega_{t}}^{2}+\left|\varrho_{\sigma}\right|_{3,1, \Omega_{t}}^{2}+\left|\vartheta_{0 t}\right|_{3,1, \Omega_{t}}^{2}+\left\|\vartheta_{0 x}\right\|_{3, \Omega_{t}}^{2}+\|\vartheta\|_{0, \Omega_{t}}^{2}+\left\|\bar{\varrho}_{\Omega_{t}}\right\|_{0, \Omega_{t}}^{2} .
\end{aligned}
$$


P r o of. We use the partition of unity. Differentiating $(3.46)_{1}$ and $(3.46)_{3}$ twice with respect to $t$ and once with respect to $\xi$, multiplying the results by $\widetilde{u}_{t t \xi} A$ and $\widetilde{\gamma}_{t t \xi} A$, respectively and next integrating the result over $\widetilde{\Omega}$ yields

$$
\begin{aligned}
\frac{1}{2} \frac{d}{d t} \int_{\tilde{\Omega}} & \left(\eta \widetilde{u}_{t t \xi}^{2}+\frac{p_{\sigma \eta}}{\eta} \widetilde{\bar{\eta}}_{\Omega_{t} t t \xi}^{2}+\frac{\eta c_{v}}{\Gamma} \widetilde{\gamma}_{t t \xi}^{2}\right) A d \xi \\
& +c_{0}\left(\left\|\widetilde{u}_{t t}\right\|_{2, \tilde{\Omega}}^{2}+\left\|\widetilde{\bar{\eta}}_{\Omega_{t} t t}\right\|_{1, \tilde{\Omega}}^{2}+\left\|\widetilde{\gamma}_{t t \xi \xi}\right\|_{0, \tilde{\Omega}}^{2}\right) \\
\leq & \varepsilon\left\|\vartheta_{0 x t t t}\right\|_{0, \Omega_{t}}^{2}+C_{1}\left(|u|_{3,0, \tilde{\Omega}}^{2}+\|\gamma\|_{0, \tilde{\Omega}}^{2}+\left\|\gamma_{0 \xi}\right\|_{2, \tilde{\Omega}}^{2}+\left|\gamma_{0 t}\right|_{2,1, \tilde{\Omega}}^{2}\right. \\
& +\left\|\bar{\eta}_{\Omega_{t}}\right\|_{0, \tilde{\Omega}}^{2}+\left|\eta_{\sigma}\right|_{1,0, \tilde{\Omega}}^{2}+|\widetilde{g}|_{2,0, \tilde{\Omega}}^{2}+|\widetilde{k}|_{2,0, \tilde{\Omega}}^{2} \\
& \left.+\left\|v_{t t}\right\|_{1, \Omega_{t}}^{2}+\left\|\vartheta_{0 t t}\right\|_{1, \Omega_{t}}^{2}+|r|_{2,0, \Omega_{t}}^{2}+\left\|\theta_{1 t t}\right\|_{1, \Omega_{t}}^{2}\right) \\
& +C_{2} X_{9}\left(1+X_{9}^{2}\right) Y_{9},
\end{aligned}
$$

where we have used equation (3.52), Lemma 5.4 of [21], the Stokes problem (3.57) and the estimate

$$
\begin{aligned}
\left\|\vartheta_{0 t t t}\right\|_{0, \Omega_{t}}^{2} \leq & \varepsilon\left\|\vartheta_{0 x t t t}\right\|_{0, \Omega_{t}}^{2} \\
& +C_{1}\left(\left\|v_{t t}\right\|_{1, \Omega_{t}}^{2}+\left\|\vartheta_{0 t t}\right\|_{1, \Omega_{t}}^{2}+|r|_{2,0, \Omega_{t}}^{2}+\left\|\theta_{1 t t}\right\|_{1, \Omega_{t}}^{2}\right) \\
& +C_{2} X_{9}\left(1+X_{9}^{2}\right) Y_{9} .
\end{aligned}
$$

For boundary subdomains we have

$$
\begin{aligned}
& \frac{1}{2} \frac{d}{d t} \int_{\hat{\Omega}}\left(\widehat{\eta} \widetilde{u}_{t t \tau}^{2}+\frac{p_{\sigma \hat{\eta}}}{\widehat{\eta}} \overline{\bar{\eta}}_{\Omega_{t} t t \tau}^{2}+\frac{\widehat{\eta} c_{v}}{\widehat{\Gamma}} \widetilde{\gamma}_{t t \tau}^{2}\right) J d z \\
& +c_{0}\left(\left\|\widetilde{u}_{t t}\right\|_{2, \hat{\Omega}}^{2}+\left\|\widetilde{\gamma}_{t t \tau z}\right\|_{0, \hat{\Omega}}^{2}\right) \\
\leq & \varepsilon\left(\left\|\widetilde{u}_{t t z z}\right\|_{0, \hat{\Omega}}^{2}+\left\|\widetilde{\bar{\eta}}_{\Omega_{t} t t z}\right\|_{0, \hat{\Omega}}^{2}+\left\|\widetilde{\gamma}_{t t z z}\right\|_{0, \hat{\Omega}}^{2}+\left\|\vartheta_{0 x t t t}\right\|_{0, \Omega_{t}}^{2}\right) \\
& +C_{1}\left(\left.\left|\widehat{u}_{3,0, \hat{\Omega}}^{2}+\|\widehat{\gamma}\|_{0, \hat{\Omega}}+\left\|\widehat{\gamma}_{0 z}\right\|_{2, \hat{\Omega}}^{2}+\right| \widehat{\gamma}_{0 t}\right|_{2,0, \hat{\Omega}} ^{2}+\left\|\widehat{\bar{\eta}}_{\Omega_{t}}\right\|_{0, \hat{\Omega}}^{2}\right. \\
& +\left|\widehat{\bar{\eta}}_{\Omega_{t}}\right|_{1,0, \hat{\Omega}}^{2}+|\widetilde{g}|_{2,0, \hat{\Omega}}^{2}+|\widetilde{k}|_{2,0, \hat{\Omega}}^{2}+\left\|\widetilde{\Gamma}_{1 t t}\right\|_{2, \hat{\Omega}}^{2}+\left\|\widetilde{\Gamma}_{1 t}\right\|_{2, \hat{\Omega}}^{2} \\
& +\left\|\widetilde{\Gamma}_{1}\right\|_{2, \hat{\Omega}}^{2}+\left\|v_{t t}\right\|_{1, \Omega_{t}}^{2}+\left\|v_{t}\right\|_{2, \Omega_{t}}^{2}+\|v\|_{2, \Omega_{t}}^{2}+\left|\vartheta_{0 t}\right|_{2,0, \Omega_{t}}^{2}+\left\|\vartheta_{0 x}\right\|_{2, \Omega_{t}}^{2} \\
& \left.+\|\vartheta\|_{0, \Omega_{t}}^{2}+|r|_{2,0, \Omega_{t}}^{2}+\left\|\theta_{1 t t}\right\|_{1, \Omega_{t}}^{2}+\left\|\theta_{1 t}\right\|_{2, \Omega_{t}}^{2}+\left\|\theta_{1}\right\|_{2, \Omega_{t}}^{2}\right) \\
& +C_{2}\left(X_{9}+\int_{0}^{t}\|v\|_{4, \Omega_{t^{\prime}}}^{2} d t^{\prime}\right)\left(1+X_{9}^{2}\right) Y_{9},
\end{aligned}
$$

where we have used the boundary conditions $(3.47)_{4}$ and $(3.47)_{5}$.

Differentiating the third component of (3.64) twice with respect to $t$, 
multiplying the result by $\widetilde{\bar{\eta}}_{\Omega_{t} n t t} J$ and integrating over $\widehat{\Omega}$ implies

$$
\begin{aligned}
& \frac{1}{2} \frac{d}{d t} \int_{\hat{\Omega}} \frac{p_{\sigma \hat{\eta}}}{\widehat{\eta}} \widetilde{\bar{\eta}}_{\Omega_{t} n t t} J d z+c_{0}\left\|\widetilde{\bar{\eta}}_{\Omega_{t} n t t}\right\|_{0, \hat{\Omega}}^{2} \\
& \leq(\varepsilon+c d)\left(\left\|\widetilde{u}_{z z t t}\right\|_{0, \hat{\Omega}}^{2}+\left\|\widetilde{\bar{\eta}}_{\Omega_{t} z t t}\right\|_{0, \hat{\Omega}}^{2}\right)+\varepsilon\left\|\vartheta_{0 x t t t}\right\|_{0, \Omega_{t}}^{2} \\
& \quad+C_{1}\left(\left\|\widetilde{u}_{z \tau t t}\right\|_{0, \hat{\Omega}}^{2}+|\widehat{u}|_{3,0, \hat{\Omega}}^{2}+\left\|\widehat{\eta}_{\sigma z}\right\|_{1, \hat{\Omega}}^{2}+\left|\widehat{\eta}_{\sigma t}\right|_{1,0, \hat{\Omega}}^{2}\right. \\
& \quad+\left\|\widehat{\bar{\eta}}_{\Omega_{t}}\right\|_{0, \hat{\Omega}}^{2}+\left\|\widehat{\gamma}_{0 z}\right\|_{2, \hat{\Omega}}^{2}+\left|\widehat{\gamma}_{0 t}\right|_{2,1, \hat{\Omega}}^{2}+|\widetilde{g}|_{2,0, \hat{\Omega}}^{2}+\left\|v_{t t}\right\|_{1, \Omega_{t}}^{2} \\
& \left.\quad+\left\|\vartheta_{0 t t}\right\|_{1, \Omega_{t}}^{2}+|r|_{2,0, \Omega_{t}}^{2}+\left\|\theta_{1 t t}\right\|_{1, \Omega_{t}}^{2}\right)+C_{2} X_{9}\left(1+X_{9}^{2}\right) Y_{9}
\end{aligned}
$$

Next, differentiating the third component of (3.66) twice with respect to $t$, multiplying the result by $\widetilde{u}_{3 n t t} J$ and integrating over $\widehat{\Omega}$ implies

$$
\begin{aligned}
& \frac{1}{2} \frac{d}{d t} \int_{\hat{\Omega}} \hat{\eta}\left|\widetilde{u}_{3 n t t}\right|^{2} J d z+c_{0}\left\|\widetilde{u}_{3 n n t t}\right\|_{0, \hat{\Omega}}^{2} \\
& \leq(\varepsilon+c d)\left(\left\|\widetilde{u}_{z z t t}\right\|_{0, \hat{\Omega}}^{2}+\left\|\widetilde{\bar{\eta}}_{\Omega_{t} z t t}\right\|_{0, \hat{\Omega}}^{2}\right)+\varepsilon\left\|\widetilde{u}_{z t t t}\right\|_{0, \hat{\Omega}}^{2} \\
& \quad+C_{1}\left(\left\|\widetilde{u}_{z \tau t t}\right\|_{0, \hat{\Omega}}^{2}+\left\|\widetilde{\bar{\eta}}_{\Omega_{t} n t t}\right\|_{0, \hat{\Omega}}^{2}+|\widehat{u}|_{3,0, \hat{\Omega}}^{2}+\left\|\widehat{\eta}_{\sigma z}\right\|_{1, \hat{\Omega}}^{2}+\left|\widehat{\eta}_{\sigma t}\right|_{1,0, \hat{\Omega}}^{2}\right. \\
& \left.\quad+\left\|\widehat{\bar{\eta}}_{\Omega_{t}}\right\|_{0, \hat{\Omega}}^{2}+\left\|\widehat{\gamma}_{0 z}\right\|_{2, \hat{\Omega}}^{2}+\left|\widehat{\gamma}_{0 t}\right|_{2,1, \Omega_{t}}^{2}+\|\widehat{\gamma}\|_{0, \hat{\Omega}}^{2}+|\widetilde{g}|_{2,0, \hat{\Omega}}^{2}\right) \\
& \quad+C_{2} X_{9}\left(1+X_{9}^{2}\right) Y_{9} .
\end{aligned}
$$

From (3.68)-(3.70) we have

(3.149) $\quad\left\|\widetilde{u}_{z \tau t t}^{\prime}\right\|_{0, \hat{\Omega}}^{2}+\left\|\widetilde{\bar{\eta}}_{\Omega_{t} \tau t t}\right\|_{0, \hat{\Omega}}^{2}$

$$
\begin{aligned}
\leq & (\varepsilon+c d)\left(\left\|\widetilde{u}_{z z t t}\right\|_{0, \hat{\Omega}}^{2}+\left\|\widetilde{\bar{\eta}}_{\Omega_{t} z t t}\right\|_{0, \hat{\Omega}}^{2}+\left\|\widetilde{u}_{z t t t}\right\|_{0, \hat{\Omega}}^{2}\right) \\
& +C_{1}\left(\left\|\operatorname{div} \widetilde{u}_{t t}\right\|_{1, \hat{\Omega}}^{2}+\left.\left|\widehat{u}_{3,0, \hat{\Omega}}^{2}+\left\|\widehat{\eta}_{\sigma z}\right\|_{1, \hat{\Omega}}^{2}+\right| \widehat{\eta}_{\sigma t}\right|_{1,0, \hat{\Omega}} ^{2}\right. \\
& \left.+\left\|\widehat{\bar{\eta}}_{\Omega_{t}}\right\|_{0, \hat{\Omega}}^{2}+\left\|\widehat{\gamma}_{0 z}\right\|_{2, \hat{\Omega}}^{2}+\left|\widehat{\gamma}_{0 t}\right|_{2,1, \hat{\Omega}}^{2}+\|\widehat{\gamma}\|_{0, \hat{\Omega}}^{2}+|\widetilde{g}|_{2,0, \hat{\Omega}}^{2}\right) \\
& +C_{2} X_{9}\left(1+X_{9}^{2}\right) Y_{9} .
\end{aligned}
$$

Next, from (3.68) it follows that

(3.150) $\quad\left\|\widetilde{u}_{n n t t}^{\prime}\right\|_{0, \hat{\Omega}}^{2}$

$$
\begin{aligned}
\leq & (\varepsilon+c d)\left(\left\|\widetilde{u}_{z z t t}\right\|_{0, \hat{\Omega}}^{2}+\left\|\widetilde{\bar{\eta}}_{\Omega_{t} z t t}\right\|_{0, \hat{\Omega}}^{2}\right) \\
& +C_{1}\left(\left\|(\operatorname{div} \widetilde{u})_{, \tau t t}\right\|_{0, \hat{\Omega}}^{2}+\left\|\widetilde{\bar{\eta}}_{\Omega_{t} \tau t t}\right\|_{0, \hat{\Omega}}^{2}+|\widehat{u}|_{3,0, \hat{\Omega}}^{2}+\left\|\widehat{\eta}_{\sigma z}\right\|_{1, \hat{\Omega}}^{2}+\left|\widehat{\eta}_{\sigma t}\right|_{1,0, \hat{\Omega}}^{2}\right. \\
& \left.+\left\|\widehat{\bar{\eta}}_{\Omega_{t}}\right\|_{0, \hat{\Omega}}^{2}+\left\|\widehat{\gamma}_{0 z}\right\|_{2, \hat{\Omega}}^{2}+\left|\widehat{\gamma}_{0 t}\right|_{2,1, \hat{\Omega}}^{2}+\|\widehat{\gamma}\|_{0, \hat{\Omega}}^{2}+|\widetilde{g}|_{2,0, \hat{\Omega}}^{2}\right) \\
& +C_{2} X_{9}\left(1+X_{9}^{2}\right) Y_{9} .
\end{aligned}
$$


Dividing (3.84) by $\widehat{\Gamma}$, differentiating twice with respect to $t$, multiplying the result by $\widetilde{\gamma}_{n n t t} J$ and integrating over $\widehat{\Omega}$ we get

$$
\begin{aligned}
& \frac{1}{2} \frac{d}{d t} \int_{\hat{\Omega}} \frac{\widehat{\eta} c_{v}}{\widehat{\Gamma}} \widetilde{\gamma}_{n t t}^{2} J d z+\frac{\kappa}{\theta^{*}}\left\|\widetilde{\gamma}_{n n t t}\right\|_{0, \hat{\Omega}}^{2} \\
& \leq(\varepsilon+c d)\left(\left\|\widetilde{\gamma}_{z z t t}\right\|_{0, \hat{\Omega}}^{2}+\left\|\widetilde{\bar{\eta}}_{\Omega_{t} z z t}\right\|_{0, \hat{\Omega}}^{2}\right)+\varepsilon\left(\left\|\widetilde{\gamma}_{z t t t}\right\|_{0, \hat{\Omega}}^{2}+\left\|\vartheta_{0 x t t t}\right\|_{0, \Omega_{t}}^{2}\right) \\
& \quad+C_{1}\left(\left\|\widetilde{\gamma}_{z \tau t t}\right\|_{0, \hat{\Omega}}^{2}+\left\|\widehat{\gamma}_{0 z}\right\|_{2, \hat{\Omega}}^{2}+\left|\widehat{\gamma}_{0 t}\right|_{2,1, \hat{\Omega}}^{2}+\|\widehat{\gamma}\|_{0, \hat{\Omega}}^{2}+|\widehat{u}|_{3,1, \hat{\Omega}}^{2}\right. \\
& \quad+\left\|\widehat{\eta}_{\sigma z}\right\|_{1, \hat{\Omega}}^{2}+\left|\widehat{\eta}_{\sigma t}\right|_{1,0, \hat{\Omega}}^{2}+\left\|\widehat{\bar{\eta}}_{\Omega_{t}}\right\|_{0, \hat{\Omega}}^{2}+|\widetilde{k}|_{2,0, \hat{\Omega}}^{2} \\
& \left.\quad+\left\|v_{t t}\right\|_{1, \Omega_{t}}^{2}+\left\|\vartheta_{0 t t}\right\|_{1, \Omega_{t}}^{2}+|r|_{2,0, \Omega_{t}}^{2}+\left\|\theta_{1 t t}\right\|_{1, \Omega_{t}}^{2}\right) \\
& \quad+C_{2} X_{9}\left(1+X_{9}^{2}\right) Y_{9} .
\end{aligned}
$$

Next, using (3.137) we get

$$
\begin{aligned}
\left\|\widetilde{\gamma}_{z \tau t t}\right\|_{0, \hat{\Omega}}^{2} \leq & (\varepsilon+c d)\left(\left\|\widetilde{u}_{z z t t}\right\|_{0, \hat{\Omega}}^{2}+\left\|\widetilde{\gamma}_{z z t t}\right\|_{0, \hat{\Omega}}^{2}\right)+\varepsilon\left\|\widetilde{\gamma}_{z t t t}\right\|_{0, \hat{\Omega}}^{2} \\
& +C_{1}\left(\left.\left|\widehat{u}_{3,1, \hat{\Omega}}^{2}+\left\|\widehat{\eta}_{\sigma z}\right\|_{1, \hat{\Omega}}^{2}+\right| \widehat{\eta}_{\sigma t}\right|_{1,0, \hat{\Omega}} ^{2}+\left\|\widehat{\bar{\eta}}_{\Omega_{t}}\right\|_{0, \hat{\Omega}}^{2}\right. \\
& +\left\|\widehat{\gamma}_{0 z}\right\|_{2, \hat{\Omega}}^{2}+\left|\widehat{\gamma}_{0 t}\right|_{2,1, \hat{\Omega}}^{2}+\|\widehat{\gamma}\|_{0, \hat{\Omega}}^{2}+|\widetilde{k}|_{2,0, \hat{\Omega}}^{2} \\
& \left.+\left\|v_{t t}\right\|_{1, \Omega_{t}}^{2}+\left\|\vartheta_{0 t t}\right\|_{1, \Omega_{t}}^{2}+|r|_{2,0, \Omega_{t}}^{2}+\left\|\theta_{1 t t}\right\|_{1, \Omega_{t}}^{2}\right) \\
& +C_{2} X_{9}\left(1+X_{9}^{2}\right) Y_{9} .
\end{aligned}
$$

Finally, we have

$$
\frac{1}{2} \frac{d}{d t} \int_{\hat{\Omega}} \widehat{\eta}_{u_{z t t}}^{2} J d z \leq \varepsilon\left\|\widetilde{u}_{z t t t}\right\|_{0, \hat{\Omega}}^{2}+C_{1}\left\|\widetilde{u}_{z t t}\right\|_{0, \hat{\Omega}}^{2}
$$

and

$$
\begin{aligned}
& \frac{1}{2} \frac{d}{d t} \int_{\hat{\Omega}} \frac{\widehat{\eta} c_{v}}{\widehat{\Gamma}} \widetilde{\gamma}_{z t t}^{2} J d z \leq \varepsilon\left(\left\|\widetilde{\gamma}_{z t t t}\right\|_{0, \hat{\Omega}}^{2}+\left\|\vartheta_{0 x t t t}\right\|_{0, \hat{\Omega}}^{2}\right) \\
& \quad+C_{1}\left(\left\|\widehat{\gamma}_{0 z z}\right\|_{0, \hat{\Omega}}^{2}+\left\|v_{t t}\right\|_{1, \Omega_{t}}^{2}+\left\|\vartheta_{0 t t}\right\|_{1, \Omega_{t}}^{2}+|r|_{2,0, \Omega_{t}}^{2}+\left\|\theta_{1 t t}\right\|_{1, \Omega_{t}}^{2}\right) \\
& \quad+C_{2} X_{9}\left(1+X_{9}^{2}\right) Y_{9} .
\end{aligned}
$$

By estimates (3.146)-(3.154) we get

$$
\begin{aligned}
& \quad \frac{1}{2} \frac{d}{d t} \int_{\hat{\Omega}}\left(\widehat{\eta} \widetilde{u}_{z t t}^{2}+\frac{p_{\sigma \hat{\eta}}}{\hat{\eta}} \widetilde{\eta}_{\Omega_{t} z t t}^{2}+\frac{\widehat{\eta} c_{v}}{\widehat{\Gamma}} \widetilde{\gamma}_{z t t}^{2}\right) J d z \\
& +c_{0}\left(\left\|\widetilde{u}_{t t}\right\|_{2, \hat{\Omega}}^{2}+\left\|\widetilde{\bar{\eta}}_{\Omega_{t} t t}\right\|_{1, \hat{\Omega}}^{2}+\left\|\widetilde{\gamma}_{z z t t}\right\|_{0, \hat{\Omega}}^{2}\right) \\
& \leq \varepsilon\left(\left\|\widetilde{u}_{t t z z}\right\|_{0, \hat{\Omega}}^{2}+\left\|\widetilde{\bar{\eta}}_{\Omega_{t} t t z}\right\|_{0, \hat{\Omega}}^{2}+\left\|\widetilde{\gamma}_{t t z z}\right\|_{0, \hat{\Omega}}^{2}\right.
\end{aligned}
$$




$$
\begin{aligned}
& \left.+\left\|\widetilde{u}_{z t t t}\right\|_{0, \hat{\Omega}}^{2}+\left\|\widetilde{\gamma}_{z t t t}\right\|_{0, \hat{\Omega}}^{2}+\left\|\vartheta_{0 x t t t}\right\|_{0, \Omega_{t}}^{2}\right) \\
& +C_{1}\left(|\widehat{u}|_{3,0, \hat{\Omega}}^{2}+\|\widehat{\gamma}\|_{0, \hat{\Omega}}^{2}+\left\|\widehat{\gamma}_{0 z}\right\|_{2, \hat{\Omega}}^{2}+\left|\widehat{\gamma}_{0 t}\right|_{2,0, \hat{\Omega}}^{2}+\left\|\widehat{\bar{\eta}}_{\Omega_{t}}\right\|_{0, \hat{\Omega}}^{2}\right. \\
& +\left|\widehat{\bar{\eta}}_{\Omega_{t}}\right|_{1,0, \Omega_{t}}^{2}+|\widetilde{g}|_{2,0, \hat{\Omega}}^{2}+|\widetilde{k}|_{2,0, \hat{\Omega}}^{2}+\left\|\widetilde{\Gamma}_{1}\right\|_{2, \hat{\Omega}}^{2}+\left\|\widetilde{\Gamma}_{1 t}\right\|_{2, \hat{\Omega}}^{2} \\
& +\left\|\widetilde{\Gamma}_{1 t t}\right\|_{2, \hat{\Omega}}^{2}+\left\|v_{t t}\right\|_{1, \Omega_{t}}^{2}+\|v\|_{2, \Omega_{t}}^{2}+\left|v_{t}\right|_{2,1, \Omega_{t}}^{2}+\left|\vartheta_{0 t}\right|_{2,0, \Omega_{t}}^{2}+\left\|\vartheta_{0 x}\right\|_{2, \Omega_{t}}^{2} \\
& \left.+\|\vartheta\|_{0, \Omega_{t}}^{2}+|r|_{2,0, \Omega_{t}}^{2}+\left\|\theta_{1 t t}\right\|_{1, \Omega_{t}}^{2}+\left\|\theta_{1 t}\right\|_{2, \Omega_{t}}^{2}+\left\|\theta_{1}\right\|_{2, \Omega_{t}}^{2}\right) \\
& +C_{2}\left(X_{9}+\int_{0}^{t}\|v\|_{4, \Omega_{t^{\prime}}}^{2} d t^{\prime}\right)\left(1+X_{9}^{2}\right) Y_{9} .
\end{aligned}
$$

Inequalities (3.144) and (3.155) yield the assertion of the lemma.

LEMMA 3.12. Let $v, \varrho, \vartheta_{0}$ be a sufficiently smooth solution of problem (3.3). Then

$$
\begin{aligned}
\frac{1}{2} \frac{d}{d t} \int_{\Omega_{t}}\left(\varrho v_{t t t}^{2}\right. & \left.+\frac{p_{\sigma \varrho}}{\varrho} \varrho_{\sigma t t t}^{2}+\frac{\varrho c_{v}}{\theta} \vartheta_{0 t t t}^{2}\right) d x \\
& +c_{0}\left(\left\|v_{t t t}\right\|_{1, \Omega_{t}}^{2}+\left\|\varrho_{\sigma t t t}\right\|_{0, \Omega_{t}}^{2}+\left\|\vartheta_{0 t t t}\right\|_{1, \Omega_{t}}^{2}\right) \\
\leq & C_{1}\left(\left\|v_{t t}\right\|_{1, \Omega_{t}}^{2}+\left\|\vartheta_{0 t t}\right\|_{1, \Omega_{t}}^{2}+\left\|f_{t t t}\right\|_{0, \Omega_{t}}^{2}+|f|_{2,0, \Omega_{t}}^{2}\right. \\
& \left.+\left\|r_{t t t}\right\|_{0, \Omega_{t}}^{2}+|r|_{2,0, \Omega_{t}}^{2}+\left|\theta_{1}\right|_{4,1, \Omega_{t}}^{2}\right) \\
& +C_{2} X_{10}\left(1+X_{10}^{3}\right) Y_{10}
\end{aligned}
$$

where

$X_{10}=|v|_{3,0, \Omega_{t}}^{2}+\left|\varrho_{\sigma}\right|_{3,0, \Omega_{t}}^{2}+\left|\vartheta_{0}\right|_{3,0, \Omega_{t}}^{2}, \quad Y_{10}=|v|_{4,1, \Omega_{t}}^{2}+\left|\varrho_{\sigma}\right|_{3,0, \Omega_{t}}^{2}+\left|\vartheta_{0}\right|_{4,1, \Omega_{t}}^{2}$.

Proof. Differentiating (3.3) 1 three times with respect to $t$, multiplying the result by $v_{t t t}$ and integrating over $\Omega_{t}$ yields

$$
\begin{aligned}
\frac{1}{2} \frac{d}{d t} \int_{\Omega_{t}} \varrho v_{t t t}^{2} d x+\frac{1}{2} \mu E_{\Omega_{t}}\left(v_{t t t}\right)+(\nu-\mu)\left\|\operatorname{div} v_{t t t}\right\|_{0, \Omega_{t}}^{2} \\
\quad-\int_{\Omega_{t}} p_{\sigma \varrho} \varrho_{\sigma t t t} \operatorname{div} v_{t t t} d x-\int_{\Omega_{t}} p_{\varrho \theta} \vartheta_{0 t t t} \operatorname{div} v_{t t t} d x \\
\quad-\int_{S_{t}}\left(n_{i} T^{i j}\left(v, \varrho_{\sigma}\right)\right)_{, t t t} v_{i t t t} d s \\
\leq \varepsilon\left(\left\|v_{t t t}\right\|_{1, \Omega_{t}}^{2}+\left\|\varrho_{\sigma t t t}\right\|_{0, \Omega_{t}}^{2}\right) \\
\quad+C_{1}\left(|f|_{2,0, \Omega_{t}}^{2}+\left\|f_{t t t}\right\|_{0, \Omega_{t}}^{2}\right)+C_{2} X_{10}\left(1+X_{10}^{3}\right) Y_{10} .
\end{aligned}
$$


Next, dividing $(3.3)_{3}$ by $\theta$, differentiating three times with respect to $t$, multiplying by $\vartheta_{0 t t t}$ and integrating over $\Omega_{t}$ gives

$$
\begin{aligned}
& \frac{1}{2} \frac{d}{d t} \int_{\Omega_{t}} \frac{\varrho c_{v}}{\theta} \vartheta_{0 t t t}^{2} d x+\frac{\kappa}{\theta^{*}} \int_{\Omega_{t}}\left|\nabla \vartheta_{0 t t t}\right|^{2} d x \\
& \quad+\int_{\Omega_{t}} p_{\varrho \theta} \vartheta_{0 t t t} \operatorname{div} v_{t t t} d x-\int_{S_{t}}\left(\frac{n \cdot \nabla \vartheta_{0}}{\theta}\right)_{, t t t} \vartheta_{0 t t t} d s \\
& \leq \varepsilon\left(\left\|v_{t t t}\right\|_{1, \Omega_{t}}^{2}+\left\|\varrho_{\sigma t t t}\right\|_{0, \Omega_{t}}^{2}+\left\|\vartheta_{0 t t t}\right\|_{1, \Omega_{t}}^{2}\right) \\
& \quad+C_{1}\left(|r|_{2,0, \Omega_{t}}^{2}+\left\|r_{t t t}\right\|_{0, \Omega_{t}}^{2}+\left\|\theta_{1 t t t}\right\|_{1, \Omega_{t}}\right)+C_{2} X_{10}\left(1+X_{10}^{3}\right) Y_{10}
\end{aligned}
$$

Now, using the continuity equation $(3.3)_{2}$, Lemma 5.5 of [21], inequality (3.145) and the estimate

$$
\left\|\varrho_{\sigma t t t}\right\|_{0, \Omega_{t}}^{2} \leq c\left\|v_{t t}\right\|_{1, \Omega_{t}}^{2}+C_{2} X_{10}\left(1+X_{10}^{2}\right) Y_{10}
$$

we obtain (3.156).

Estimating $\|\vartheta\|_{0, \Omega_{t}}^{2}+\left\|\bar{\varrho}_{\Omega_{t}}\right\|_{0, \Omega_{t}}^{2}$ by $\left\|\vartheta_{0 x}\right\|_{0, \Omega_{t}}^{2}+\left\|p_{\sigma}\right\|_{0, \Omega_{t}}^{2}$ (by using (3.19) and $(3.27)$ ) from the above lemmas for sufficiently small $\varepsilon$ we obtain

THEOREM 3.13. For a sufficiently smooth solution $v, \varrho, \vartheta_{0}$ of (3.3) we have

$$
\frac{d \bar{\phi}}{d t}+c_{0} \Phi \leq c_{1} P(\phi)\left(\phi+\int_{0}^{t}\|v\|_{4, \Omega_{t^{\prime}}}^{2} d t^{\prime}\right)\left(1+\phi^{3}\right)(\phi+\Phi)+c_{2} F+c_{3} \Psi
$$

where

$$
\begin{aligned}
\bar{\phi}(t)= & \int_{\Omega_{t}} \varrho \sum_{0 \leq|\alpha|+i \leq 3}\left|D_{x}^{\alpha} \partial_{t}^{i} v\right|^{2} d x \\
& +\int_{\Omega_{t}}\left(\frac{p_{1}}{\varrho} \varrho_{\sigma}^{2}+\bar{\varrho}_{\Omega_{t}}^{2}+\frac{p_{2} \varrho c_{v}}{p_{\theta} \theta} \vartheta_{0}^{2}\right) d x \\
& +\int_{\Omega_{t}} \frac{p_{\sigma \varrho}}{\varrho} \sum_{1 \leq|\alpha|+i \leq 3}\left|D_{x}^{2} \partial_{t}^{i} \varrho_{\sigma}\right|^{2} d x \\
& +\int_{\Omega_{t}} \frac{\varrho c_{v}}{\theta} \sum_{1 \leq|\alpha|+i \leq 3}\left|D_{x}^{\alpha} \partial_{t}^{i} \vartheta_{0}\right|^{2} d x, \\
\phi(t)= & |v|_{3,0, \Omega_{t}}^{2}+\left|\varrho_{\sigma}\right|_{3,0, \Omega_{t}}^{2}+\left|\vartheta_{0}\right|_{3,0, \Omega_{t}}^{2}+\left\|\bar{\varrho}_{\Omega_{t}}\right\|_{0, \Omega_{t}}^{2}, \\
\Phi(t)= & |v|_{4,1, \Omega_{t}}^{2}+\left|\varrho_{\sigma}\right|_{3,0, \Omega_{t}}^{2}-\left\|\varrho_{\sigma}\right\|_{0, \Omega_{t}}^{2}+\left\|\bar{\varrho}_{\Omega_{t}}\right\|_{0, \Omega_{t}}^{2} \\
& +\left|\vartheta_{0}\right|_{4,1, \Omega_{t}}^{2}-\left\|\vartheta_{0}\right\|_{0, \Omega_{t}}^{2}+\|\vartheta\|_{0, \Omega_{t}}^{2}, \\
F(t)= & \left\|f_{t t t}\right\|_{0, \Omega_{t}}^{2}+|f|_{2,0, \Omega_{t}}^{2}+\left\|r_{t t t}\right\|_{0, \Omega_{t}}^{2}+|r|_{2,0, \Omega_{t}}^{2} \\
& +\|r\|_{0, \Omega_{t}}+\left|\theta_{1}\right|_{4,1, \Omega_{t}}^{2}+\left\|\theta_{1}\right\|_{1, \Omega_{t}}, \\
\Psi(t)= & \|v\|_{0, \Omega_{t}}^{2}+\left\|p_{\sigma}\right\|_{0, \Omega_{t}}^{2},
\end{aligned}
$$


$P$ is an increasing continuous function; $c_{0}<1$ is a positive constant depending on $\varrho_{*}, \varrho^{*}, \theta_{*}, \theta^{*}, \mu, \nu, \kappa ;$ and $c_{i}(i=1,2,3)$ are positive constants depending on $\varrho_{*}, \varrho^{*}, \theta_{*}, \theta^{*}, \int_{0}^{t}\|v\|_{3, \Omega_{t^{\prime}}} d t^{\prime},\|S\|_{4-1 / 2}, T$ and constants from the imbedding Lemma 2.1 and the Korn inequalities (see Section 5 of [21]).

\section{References}

[1] O. V. Besov, V. P. Il'in and S. M. Nikol'skiŭ, Integral Representation of Functions and Imbedding Theorems, Nauka, Moscow, 1975 (in Russian).

[2] L. Landau and E. Lifschitz, Mechanics of Continuum Media, Nauka, Moscow, 1984; new edition: Hydrodynamics, Nauka, Moscow, 1986 (in Russian).

[3] A. Matsumura and T. Nishida, The initial value problem for the equations of motion of viscous and heat-conductive gases, J. Math. Kyoto Univ. 20 (1980), 67104.

[4] - - - The initial value problem for the equations of motion of compressible viscous and heat-conductive fluids, Proc. Japan Acad. Ser. A 55 (1979), 337-342.

[5] - - - The initial boundary value problem for the equations of motion of compressible viscous and heat-conductive fluids, preprint of Univ. of Wisconsin, MRC Technical Summary Report no. 2237, 1981.

[6] - - - Initial boundary value problems for the equations of motion of general fluids, in: Computing Methods in Applied Sciences and Engineering, V. R. Glovinski and J. L. Lions (eds.), North-Holland, Amsterdam, 1982.

[7] - - - Initial boundary value problems for the equations of motion of compressible viscous and heat-conductive fluids, Comm. Math. Phys. 89 (1983), 445-464.

[8] K. Pileckas and W. M. Zajączkowski, On the boundary problem for stationary compressible Navier-Stokes equations, ibid. 128 (1990), 1-36.

[9] V. A. Solonnikov, On an unsteady flow of a finite mass of a liquid bounded by a free surface, Zap. Nauchn. Sem. LOMI 152 (1986), 137-157 (in Russian); English transl.: J. Soviet Math. 10 (1988), 672-686.

[10] - Solvability of the evolution problem for an isolated mass of a viscous incompressible capillary liquid, Zap. Nauchn. Sem. LOMI 140 (1984), 179-186 (in Russian); English transl.: J. Soviet Math. 33 (1986), 223-238.

[11] _- On unsteady motion of an isolated volume of a viscous incompressible fluid, Izv. Akad. Nauk SSSR Ser. Mat. 51 (1987), 1065-1087 (in Russian).

[12] V. A. Solonnikov and A. Tani, Evolution free boundary problem for equations of motion of viscous compressible barotropic liquids, preprint of Paderborn University.

[13] A. Valli, Periodic and stationary solutions for compressible Navier-Stokes equations via a stability method, Ann. Scuola Norm. Sup. Pisa (4) 10 (1983), 607-647.

[14] A. Valli and W. M. Zajączkowski, Navier-Stokes equations for compressible fluids: global existence and qualitative properties of the solution in the general case, Comm. Math. Phys. 103 (1986), 259-296.

[15] E. Zadrzyńska and W. M. Zajączkowski, On local motion of a general compressible viscous heat conducting fluid bounded by a free surface, Ann. Polon. Math. 59 (1994), 133-170.

[16] _- - - On global motion of a compressible heat conducting fluid bounded by a free surface, Acta Appl. Math., to appear. 
[17] E. Zadrzyńska and W. M. Zajączkowski, Conservation laws in free boundary problems for viscous compressible heat conducting fluids, Bull. Polish Acad. Sci. Tech. Sci. 42 (1994), 197-207.

[18] - - - Conservation laws in free boundary problems for viscous compressible heat conducting capillary fluids, to appear.

[19] —, On a differential inequality for equations of a viscous compressible heat conducting capillary fluid bounded by a free surface, to appear.

[20] - - - On the global existence theorem for a free boundary problem for equations of a viscous compressible heat conducting fluid, Inst. Math., Pol. Acad. Sci., Prepr. 523 (1994), 1-22.

[21] - - - On the global existence theorem for a free boundary problem for equations of a viscous compressible heat conducting capillary fluid, to appear.

[22] W. M. Zajączkowski, On nonstationary motion of a compressible barotropic viscous fluid bounded by a free surface, Dissertationes Math. 324 (1993).

[23] - On local motion of a compressible viscous fluid bounded by a free surface, in: Partial Differential Equations, Banach Center Publ. 27, Inst. Math., Polish Acad. Sci., Warszawa, 1992, 511-553.

[24] —, Existence of local solutions for free boundary problems for viscous compressible barotropic fluids, Ann. Polon. Math. 60 (1995), 255-287.

[25] - On nonstationary motion of a compressible barotropic viscous capillary fluid bounded by a free surface, SIAM J. Math. Anal. 25 (1994), 1-84.

INSTITUTE OF MATHEMATICS

AND OPERATIONS RESEARCH

MILITARY UNIVERSITY OF TECHNOLOGY

S. KALISKIEGO 2

01-489 WARSZAWA, POLAND
INSTITUTE OF MATHEMATICS POLISH ACADEMY OF SCIENCES ŚNIADECKICH 8 